\title{
A Coarea Formula for Smooth CONTACT MAPPINGS OF CARnot-Carathéodory SPACES
}

\author{
M. Karmanova, S. Vodopyanov ${ }^{*}$
}

June 24, 2018

\begin{abstract}
We prove the coarea formula for sufficiently smooth contact mappings of Carnot manifolds. In particular, we investigate level surfaces of these mappings, and compare Riemannian and sub-Riemannian measures on them. Our main tool is the sharp asymptotic behavior of the Riemannian measure of the intersection of a tangent plane to a level surface and a sub-Riemannian ball. This calculation in particular implies that the sub-Riemannian measure of the set of characteristic points (i.e., the points at which the sub-Riemannian differential is degenerate) equals zero on almost every level set.
\end{abstract}

* Mathematics Subject Classification (2000): Primary 51F99; Secondary 53B99, 58A99 Keywords: Carnot manifold, differentiability, coarea formula, level set

†The research was partially supported by RFBR (Grants 10-01-00662; 11-01-00819), and the State Maintenance Program for the Leading Scientific Schools of the Russian Federation (Grant No. NSh-6613.2010.1). 


\section{Contents}

1 Introduction

2 Preliminaries 7

3 Properties of Level Sets 18

4 The Characteristic Set

5 The Degeneration Set 49

6 The Coarea Formula 


\section{Introduction}

This article is devoted to a sub-Riemannian analog

$$
\int_{\mathbb{M}} \mathcal{J}_{\widetilde{N}}^{S R}(\varphi, x) d \mathcal{H}^{\nu}(x)=\int_{\widetilde{\mathbb{M}}} d \mathcal{H}^{\tilde{\nu}}(t) \int_{\varphi^{-1}(t)} d \mathcal{H}^{\nu-\tilde{\nu}}(u)
$$

of the well-known coarea formula (1.2). Here $\varphi: \mathbb{M} \rightarrow \widetilde{\mathbb{M}}$ is a smooth contact mapping of Carnot-Carathéodory spaces, and $\mathcal{J}_{\widetilde{N}}^{S R}(\varphi, x)$ is a subRiemannian coarea factor determined by the values of a sub-Riemannian differential, or hc-differential. In some sense, we may regard (1.1) as a generalization of the results of [Pa, $\mathrm{He}, \mathrm{Mg} 1, \mathrm{Mg} 2]$ and some other articles.

It is well known that the coarea formula

$$
\int_{U} \mathcal{J}_{k}(\varphi, x) d x=\int_{\mathbb{R}^{k}} d z \int_{\varphi^{-1}(z)} d \mathcal{H}^{n-k}(u)
$$

where $\mathcal{J}_{k}(\varphi, x)=\sqrt{\operatorname{det}\left(D \varphi(x) D \varphi^{*}(x)\right)}$, has many applications in analysis on Euclidean spaces [Kr, Fe1, [Fe2, EG, GMS, LiYa, Ot]. Here we assume that $\varphi \in C^{1}\left(U, \mathbb{R}^{k}\right)$ with $U \subset \mathbb{R}^{n}$ for $n \geq k$. In particular, (1.2) applies in the theory of exterior forms and currents, and in problems about minimal surfaces (see, for example, [FeFl]). Also, Stokes' formula easily follows from the coarea formula (see, for instance, Vod1]). The development of analysis on more general structures raises a natural question of extending the coarea formula to objects with a more general geometry than in Euclidean spaces, especially to metric spaces and sub-Riemannian manifolds. In 1999, L. Ambrosio and B. Kirchheim AmKi proved an analog of the coarea formula for Lipschitz mappings defined on an $\mathcal{H}^{n}$-rectifiable metric space with values in $\mathbb{R}^{k}$ for $n \geq k$. In 2004, this formula was established for Lipschitz mappings defined on an $\mathcal{H}^{n}$-rectifiable metric space with values in an $\mathcal{H}^{k}$-rectifiable metric space for $n \geq k$ [Km1, Km4]. Moreover, conditions were found on the image and preimage of a Lipschitz mapping defined on an $\mathcal{H}^{n}$-rectifiable metric space with values in an arbitrary metric space which are necessary and sufficient for the validity of the coarea formula. Independently of that result, the level sets of those mappings were investigated, and the metric analog of implicit function theorem was proved [Km2, $\mathrm{Km} 4, \mathrm{Km} 6$ ].

All results mentioned above were obtained on rectifiable metric spaces. Note that their metric structure is similar to that of Riemannian manifolds. However, there exist non-rectifiable metric spaces whose geometry is not comparable to the Riemannian one. Carnot manifolds are of special interest (for results on their non-rectifiability, see, for instance, AmKi]). Sub-Riemannian 
geometry naturally arises in the theory of subelliptic equations, contact geometry, optimal control theory, non-holonomic mechanics, neurobiology, and other areas (see, e. g., NSW, Gr, Be, Mg1, MrMo1, MrMo2, AgMa, Je, VodG, Vod2, Vod3, CS, HlPa $)$. This theory has many applications. In addition, it has many well-known unsolved problems.

One of them is the problem of the sub-Riemannian coarea formula, which is useful for developing a non-holonomic theory of currents, exterior forms, extremal surfaces (in sub-Riemannian and sub-Lorentzian geometries), and so forth.

Heisenberg groups and Carnot groups are well-known particular cases of Carnot manifolds. In 1982, P. Pansu proved the coarea formula for realvalued functions defined on a Heisenberg group [Pa]. Next, J. Heinonen [He] extended this formula to smooth functions defined on a Carnot group. Another result concerning the analog of (1.2) belongs to V. Magnani. In 2000, he proved a coarea inequality for mappings of Carnot groups [Mg1]. The equality was established for a mapping from a Heisenberg group to the Euclidean space $\mathbb{R}^{k} \mathrm{Mg} 2$. The validity of coarea formula even for a model case of a mapping from a Carnot group to other Carnot group has remained an open question.

The purpose of this paper is to prove the coarea formula for sufficiently smooth contact mappings $\varphi: \mathbb{M} \rightarrow \widetilde{\mathbb{M}}$ of Carnot manifolds. We emphasize that all results are new even in the particular case of a mapping of Carnot groups.

As we mentioned above, for the first time a non-holonomic analogue of the coarea formula was discovered by P. Pansu [Pa. One of the basic ideas was to prove the sub-Riemannian coarea formula via the Riemannian one:

$$
\begin{aligned}
(1.2)) & \Rightarrow \int_{U} \mathcal{J}_{\widetilde{N}}^{S R}(\varphi, x) d \mathcal{H}^{\nu}(x) \\
= & \int_{\widetilde{\mathbb{M}}} d \mathcal{H}^{\tilde{\nu}}(z) \int_{\varphi^{-1}(z)} \frac{\mathcal{J}_{\widetilde{N}}^{S R}(\varphi, u)}{\mathcal{J}_{\widetilde{N}}(\varphi, x)} d \mathcal{H}^{N-\widetilde{N}}(u) \stackrel{?}{=} \int_{\widetilde{\mathbb{M}}} d \mathcal{H}^{\tilde{\nu}}(z) \int_{\varphi^{-1}(z)} d \mathcal{H}^{\nu-\tilde{\nu}}(u)
\end{aligned}
$$

Here $N$ and $\widetilde{N}$ are the topological dimensions while $\nu$ and $\tilde{\nu}$ are the Hausdorff dimensions of the preimage and image respectively. It is clear that in subRiemannian spaces these dimensions differ. Other authors subsequently used the same idea.

It follows easily from (1.3) that the crucial point in this method is to understand the relation between the Riemannian and sub-Riemannian measures on the Carnot manifolds themselves and on the level sets. Moreover, 
an appropriate definition of the sub-Riemannian coarea factor is required. It is well known that the question of measure relation on the given spaces is trivial, whereas those of the geometry of level sets and the sub-Riemannian coarea factor are quite complicated. The main difficulties are related to the peculiarities of sub-Riemannian metrics. The inequivalence of Riemannian and sub-Riemannian metrics is illustrated for example by the fact that the Riemannian distance from the center of a radius $r$ sub-Riemannian ball to its boundary varies from $r$ to $r^{M}, M>1$, where the constant $M$ depends on the structure of the space. Thus, an immediate question arises: how "sharply" does the tangent plane approximate a level set? (This question is important since the Riemannian tangency order $o(r)$ is insufficient here: a level surface can "jump" out of a sub-Riemannian ball much earlier than necessary if this order of tangency is higher than $o(r)$.) Also, it is a problem whether or not there exists a metric allowing us to describe the shape and geometry of the intersection of a sub-Riemannian ball and a level surface. Even if we answer these two questions, the most difficult question concerns the relation between the Hausdorff dimensions of the image and the intersection of a ball and a level set.

In this article we solve problems stated above. Firstly, we divide the set of all points at which the classical differential is non-degenerate into a regular set and a characteristic set. Next, we define a sub-Riemannian quasimetric $d_{2}$ that enables us to calculate the measure of the intersection of a sub-Riemannian ball and a tangent plane (see below Remark 3.8 for details). A crucial idea in the construction of $d_{2}$ is based on the fact that a ball in this quasimetric is asymptotically equal to direct product of Euclidean balls:

$$
B_{d_{2}}^{N}(x, r) \approx B^{n_{1}}(x, r) \times B^{n_{2}}\left(x, r^{2}\right) \times \ldots \times B^{n_{M}}\left(x, r^{M}\right), \quad M>1,
$$

where $N$ is the topological dimension of a Carnot manifold, and $n_{i}$ are the (topological) dimensions of the Euclidean balls $B^{n_{i}}, i=1, \ldots, M$. Thus, if a plane intersects a ball of this type then we can easily determine the shape of the intersection (contrary to the case of a "box" quasimetric with

$$
\operatorname{Box}(x, r) \approx Q^{n_{1}}(x, r) \times Q^{n_{2}}\left(x, r^{2}\right) \times \ldots \times Q^{n_{M}}\left(x, r^{M}\right),
$$

where we have Euclidean cubes $Q^{n_{i}}, i=1, \ldots, M$, instead of balls, since the cubes have sections of different shapes). Studying the sharpness of the approximation to a level surface by its tangent plane, we introduce a "mixed" quasimetric possessing both Riemannian and sub-Riemannian properties. We prove that at regular points the tangent plane approximates the level surface sufficiently well, and this fact enables us to calculate the (Riemannian) 
measure of this intersection, which depends on the Hausdorff dimensions of both the image and preimage. In other words, it is equivalent to $r^{\nu-\tilde{\nu}}$ (see Theorems 3.7 and 3.11). These results yield analytic expression of the relation between the Riemannian and sub-Riemannian measures at the regular points on level sets (see Theorem 3.17).

The characteristic set case is a little more complicated since precisely near a characteristic point a surface can "jump" out of a sub-Riemannian ball. For this reason we cannot estimate the measure of the intersection of a ball and a surface via the measure of the intersection of a ball and the tangent plane at a characteristic point. Note also that in all papers mentioned above the preimage has a group structure, whose properties are essential for proving that the measure of the set of all characteristic points on each level surface equals zero. In the case of a mapping of two Carnot manifolds, both the image and preimage lack a group structure, and the approximation of a manifold by its local Carnot group is insufficient for the extension of the methods developed to our case. That is why we create an "intrinsic" method for studying the properties of the characteristic set.

The result on the characteristic set is stated in Theorem 4.1,

In Section 4 we prove that the degenerate set of the classical differential does not affect either part of the coarea formula.

Finally, in Section 5, we define the sub-Riemannian coarea factor (via the values of the $h c$-differential) and derive the sub-Riemannian coarea formula (1.1).

For mappings $\varphi: \mathbb{H}^{1} \rightarrow \mathbb{R}$, formula (1.1) becomes

$$
\int_{\mathbb{M}}\left|\nabla_{H} \varphi(x)\right| \cdot \frac{\omega_{3}^{2}}{\omega_{4} \cdot 4} d \mathcal{H}^{\nu}(x)=\int_{\widetilde{\mathbb{M}}} d \mathcal{H}^{\tilde{\nu}}(t) \int_{\varphi^{-1}(t)} d \mathcal{H}^{\nu-\tilde{\nu}}(u)
$$

since in this case we have

$$
\mathcal{J}_{\widetilde{N}}^{S R}(\varphi, x)=\left|\nabla_{H} \varphi(x)\right| \cdot \frac{\omega_{3}^{2}}{\omega_{4} \cdot 4} .
$$

Here $\nabla_{H} \varphi(x)$ is the "horizontal part" of the gradient of $\varphi$ at $x$, and the coefficient $\frac{\omega_{3}^{2}}{\omega_{4} \cdot 4}$ appears due to the choice of a constant factor in the definition of the Hausdorff measure (to define $\mathcal{H}^{\mu}$ we set this factor to $\omega_{\mu}$, and if we consider $2^{\mu}$ instead of $\omega_{\mu}$ then this factor will be equal to 1$)$; cf. [Pa]. For 
$\varphi: \mathbb{H}^{n} \rightarrow \mathbb{R}^{k}, k \leq 2 n$, we deduce that

$$
\begin{aligned}
\int_{\mathbb{M}} \sqrt{\operatorname{det}\left(D_{H} \varphi(x) D_{H} \varphi(x)^{*}\right)} \cdot \frac{\omega_{2 n+1} \cdot \omega_{2 n+2-k}}{\omega_{2 n+2} \cdot \omega_{2 n-k} \cdot 2} d \mathcal{H}^{\nu}(x) & \\
& =\int_{\tilde{\mathbb{M}}} d \mathcal{H}^{\tilde{\nu}}(t) \int_{\varphi^{-1}(t)} d \mathcal{H}^{\nu-\tilde{\nu}}(u)
\end{aligned}
$$

and

$$
\mathcal{J}_{\widetilde{N}}^{S R}(\varphi, x)=\sqrt{\operatorname{det}\left(D_{H} \varphi(x) D_{H} \varphi(x)^{*}\right)} \cdot \frac{\omega_{2 n+1} \cdot \omega_{2 n+2-k}}{\omega_{2 n+2} \cdot \omega_{2 n-k} \cdot 2} .
$$

Here $D_{H} \varphi(x)$ is the "horizontal part" of the differential $D \varphi(x)$, and if we replace all occurrences of $\omega_{\mu}$ by $2^{\mu}$ then the coefficient following the Gram determinant of $D_{H} \varphi(x)$ will be equal to 1 ; see also Mg2. Similarly, for $\mathbb{M}=\mathbb{G}$ and $\widetilde{\mathbb{M}}=\mathbb{R}$ we obtain

$$
\int_{\mathbb{M}}\left|\nabla_{H} \varphi(x)\right| \cdot \frac{\omega_{N} \cdot \omega_{\nu-1}}{\omega_{\nu} \cdot \prod_{k=2}^{M} \omega_{n_{k}} \cdot \omega_{n_{1}-1}} d \mathcal{H}^{\nu}(x)=\int_{\widetilde{\mathbb{M}}} d \mathcal{H}^{\tilde{\nu}}(t) \int_{\varphi^{-1}(t)} d \mathcal{H}^{\nu-\tilde{\nu}}(u)
$$

and

$$
\mathcal{J}_{\widetilde{N}}^{S R}(\varphi, x)=\left|\nabla_{H} \varphi(x)\right| \cdot \frac{\omega_{N} \cdot \omega_{\nu-1}}{\omega_{\nu} \cdot \prod_{k=2}^{M} \omega_{n_{k}} \cdot \omega_{n_{1}-1}} .
$$

Once again, it is easy to see that if we take $2^{\mu}$ instead of $\omega_{\mu}$ then the coefficient following the length of the horizontal gradient of $\varphi$ at $x$ will be equal to 1 ; compare with [He].

Thus, for sufficiently smooth mappings we obtain all previous results as particular cases.

\section{Preliminaries}

In this section we introduce some necessary definitions and mention important facts used to prove the main result.

Definition 2.1 (cf. [Gr, KmVod, $[\mathrm{NSW}]$ ). Fix a connected Riemannian $C^{\infty}$ manifold $\mathbb{M}$ of topological dimension $N$. The manifold $\mathbb{M}$ is called a CarnotCarathéodory space if the tangent bundle $T \mathbb{M}$ has a filtration

$$
H \mathbb{M}=H_{1} \mathbb{M} \subsetneq \ldots \subsetneq H_{i} \mathbb{M} \subsetneq \ldots \subsetneq H_{M} \mathbb{M}=T \mathbb{M}
$$


by subbundles such that each point $p \in \mathbb{M}$ has a neighborhood $U \subset \mathbb{M}$ equipped with a collection of $C^{1, \alpha}$-smooth vector fields $X_{1}, \ldots, X_{N}, \alpha \in(0,1]$, enjoying the following two properties. For each $v \in U$,

(1) $H_{i} \mathbb{M}(v)=H_{i}(v)=\operatorname{span}\left\{X_{1}(v), \ldots, X_{\operatorname{dim} H_{i}}(v)\right\}$ is a subspace of $T_{v} \mathbb{M}$ of a constant dimension $\operatorname{dim} H_{i}, i=1, \ldots, M$;

(2) we have

$$
\left[X_{i}, X_{j}\right](v)=\sum_{k: \operatorname{deg} X_{k} \leq \operatorname{deg} X_{i}+\operatorname{deg} X_{j}} c_{i j k}(v) X_{k}(v)
$$

where the degree $\operatorname{deg} X_{k}$ is defined as $\min \left\{m \mid X_{k} \in H_{m}\right\}$;

Moreover, if the third condition holds then the Carnot-Carathéodory space is called the Carnot manifold:

(3) the quotient mapping $[\cdot, \cdot]_{0}: H_{1} \times H_{j} / H_{j-1} \mapsto H_{j+1} / H_{j}$ induced by the Lie bracket is an epimorphism for all $1 \leq j<M$.

The subbundle $H \mathbb{M}$ is called horizontal.

The number $M$ is called the depth of the manifold $\mathbb{M}$.

Properties of Carnot-Carathéodory spaces and Carnot manifolds under assumptions of regularity mentioned in definition 2.1 can be found in $\mathrm{KmVod}$, Km8, Km9, VodKm2, Km10,

Example. A Carnot group is an example of a Carnot manifold.

Definition 2.2. Consider the initial value problem

$$
\left\{\begin{array}{l}
\dot{\gamma}(t)=\sum_{i=1}^{N} y_{i} X_{i}(\gamma(t)), t \in[0,1], \\
\gamma(0)=x
\end{array}\right.
$$

where the vector fields $X_{1}, \ldots, X_{N}$ are $C^{1}$-smooth. Then, for the point $y=$ $\gamma(1)$ we write $y=\exp \left(\sum_{i=1}^{N} y_{i} X_{i}\right)(x)$.

The mapping $\left(y_{1}, \ldots, y_{N}\right) \mapsto \exp \left(\sum_{i=1}^{N} y_{i} X_{i}\right)(x)$ is called the exponential.

Definition 2.3. Consider $u \in \mathbb{M}$ and $\left(v_{1}, \ldots, v_{N}\right) \in B_{E}(0, r)$, where $B_{E}(0, r)$ is a Euclidean ball in $\mathbb{R}^{N}$. Define a mapping $\theta_{u}: B_{E}(0, r) \rightarrow \mathbb{M}$ as follows:

$$
\theta_{u}\left(v_{1}, \ldots, v_{N}\right)=\exp \left(\sum_{i=1}^{N} v_{i} X_{i}\right)(u) .
$$

It is known that $\theta_{u}$ is a $C^{1}$-diffeomorphism if $0<r \leq r_{u}$ for some $r_{u}>0$. The collection $\left\{v_{i}\right\}_{i=1}^{N}$ is called the normal coordinates or the coordinates of the $1^{\text {st }}$ kind (with respect to $u \in \mathbb{M}$ ) of the point $v=\theta_{u}\left(v_{1}, \ldots, v_{N}\right)$. 
Theorem 2.4 ([KmVod $]$ ). Fix $u \in \mathbb{M}$. The coefficients

$$
\bar{c}_{i j k}= \begin{cases}c_{i j k}(u) \text { of (2.1) } & \text { if } \operatorname{deg} X_{i}+\operatorname{deg} X_{j}=\operatorname{deg} X_{k}, \\ 0 & \text { otherwise }\end{cases}
$$

define a graded nilpotent Lie algebra.

We construct the Lie algebra $\mathfrak{g}^{u}$ of Theorem 2.4 as a graded nilpotent Lie algebra of vector fields $\left\{\left(\widehat{X}_{i}^{u}\right)^{\prime}\right\}_{i=1}^{N}$ on $\mathbb{R}^{N}$ such that the exponential mapping $\left(x_{1}, \ldots, x_{N}\right) \mapsto \exp \left(\sum_{i=1}^{N} x_{i}\left(\widehat{X}_{i}^{u}\right)^{\prime}\right)(0)$ is the identity [Po, BLU]. In view of the results of [FoSt], the value of $\left(\widehat{X}_{j}^{u}\right)^{\prime}(0)$ is equal to a standard vector $e_{i_{j}} \in \mathbb{R}^{N}$, where $i_{j} \neq i_{k}$ if $j \neq k, j=1, \ldots, N$. We associate to each vector field of the resulting collection an index $i$ so that $\left(\theta_{u}\right)_{*}\left\langle\left(\widehat{X}_{i}^{u}\right)^{\prime}\right\rangle(u)=X_{i}(u)$. By the construction, the vector fields $\left\{\left(\widehat{X}_{i}^{u}\right)^{\prime}\right\}_{i=1}^{N}$ satisfy

$$
\left[\left(\widehat{X}_{i}^{u}\right)^{\prime},\left(\widehat{X}_{j}^{u}\right)^{\prime}\right]=\sum_{\operatorname{deg} X_{k}=\operatorname{deg} X_{i}+\operatorname{deg} X_{j}} c_{i j k}(u)\left(\widehat{X}_{k}^{u}\right)^{\prime}
$$

everywhere on $\mathbb{R}^{N}$.

Notation 2.5. We use the following standard notation: for each $N$-dimensional multi-index $\mu=\left(\mu_{1}, \ldots, \mu_{N}\right)$, its homogeneous norm equals $|\mu|_{h}=$ $\sum_{i=1}^{N} \mu_{i} \operatorname{deg} X_{i}$

Definition 2.6. Refer as the graded nilpotent Carnot group $\mathbb{G}_{u} \mathbb{M}$ corresponding to the Lie algebra $\mathfrak{g}^{u}$ to the nilpotent tangent cone of $\mathbb{M}$ at $u \in \mathbb{M}$. We construct $\mathbb{G}_{u} \mathbb{M}$ in $\mathbb{R}^{N}$ as a groupalgebra $[\mathrm{Po}$, that is, the exponential map is the identity:

$$
\exp \left(\sum_{i=1}^{N} x_{i}\left(\widehat{X}_{i}^{u}\right)^{\prime}\right)(0)=\left(x_{1}, \ldots, x_{N}\right) .
$$

By the Baker-Campbell-Hausdorff formula, the group operation is defined so that the basis vector fields $\left(\widehat{X}_{i}^{u}\right)^{\prime}$ on $\mathbb{R}^{N}, i=1, \ldots, N$, are left-invariant [Po]: if

$$
x=\exp \left(\sum_{i=1}^{N} x_{i}\left(\widehat{X}_{i}^{u}\right)^{\prime}\right), y=\exp \left(\sum_{i=1}^{N} y_{i}\left(\widehat{X}_{i}^{u}\right)^{\prime}\right)
$$

then

$$
x \cdot y=z=\exp \left(\sum_{i=1}^{N} z_{i}\left(\widehat{X}_{i}^{u}\right)^{\prime}\right)
$$


where

$$
\begin{aligned}
z_{i} & =x_{i}+y_{i}, \quad \operatorname{deg} X_{i}=1, \\
z_{i} & =x_{i}+y_{i}+\sum_{\substack{\left|e_{l}+e_{j}\right|_{h}=2, l<j}} F_{e_{l}, e_{j}}^{i}(u)\left(x_{l} y_{j}-y_{l} x_{j}\right), \quad \operatorname{deg} X_{i}=2, \\
z_{i} & =x_{i}+y_{i}+\sum_{\substack{|\mu+\beta|_{h}=k, \mu>0, \beta>0}}^{i} F_{\mu, \beta}^{i}(u) x^{\mu} \cdot y^{\beta} \\
& =x_{i}+y_{i}+\sum_{\substack{\left|\mu+e_{l}+\beta+e_{j}\right|_{h}=k, l<j}} G_{\mu, \beta, l, j}^{i}(u) x^{\mu} y^{\beta}\left(x_{l} y_{j}-y_{l} x_{j}\right), \quad \operatorname{deg} X_{i}=k .
\end{aligned}
$$

Using the exponential mapping $\theta_{u}$, we can push forward the vector fields $\left(\widehat{X}_{i}^{u}\right)^{\prime}$ onto $\mathcal{U} \subset \mathbb{M}$ as

$$
\left[\left(\theta_{u}\right)_{*}\left\langle\left(\widehat{X}_{i}^{u}\right)^{\prime}\right\rangle\right]\left(\theta_{u}(x)\right)=D \theta_{u}(x)\left\langle\left(\widehat{X}_{i}^{u}\right)^{\prime}(x)\right\rangle
$$

and obtain the vector fields $\widehat{X}_{i}^{u}=\left(\theta_{u}\right)_{*}\left(\widehat{X}_{i}^{u}\right)^{\prime}$. Recall that $\widehat{X}_{i}^{u}(u)=X_{i}(u)$.

Definition 2.7. Associated to the Lie algebra $\left\{\widehat{X}_{i}^{u}\right\}_{i=1}^{N}$ at $u \in \mathbb{M}$, is a local homogeneous group $\mathcal{G}^{u} \mathbb{M}$. Define it so that the mapping $\theta_{u}$ is a local group isomorphism between some neighborhoods of the identity elements of the groups $\mathbb{G}_{u} \mathbb{M}$ and $\mathcal{G}^{u} \mathbb{M}$.

The canonical Riemannian structure on $\mathcal{G}^{u} \mathbb{M}$ is determined by the inner product at the identity element of $\mathcal{G}^{u} \mathbb{M}$ coinciding with that on $T_{u} \mathbb{M}$. The canonical Riemannian structure on the nilpotent tangent cone $\mathbb{G}_{u} \mathbb{M}$ is defined so that the local group isomorphism $\theta_{u}$ is an isometry.

Assumption 2.8. Henceforth we assume that the neighborhood $\mathcal{U}$ under consideration is such that $\mathcal{U} \subset \mathcal{G}^{u} \mathbb{M}$ for all $u \in \mathcal{U}$.

Definition 2.9. A curve $\gamma:[0,1] \rightarrow \mathbb{M}$ which is absolutely continuous in the Riemannian sense is called horizontal if $\dot{\gamma}(t) \in H_{\gamma(t)} \mathbb{M}$ for almost all $t \in[0,1]$ with respect to the Lebesgue measure on $[0,1]$.

A horizontal curve in $\mathcal{G}^{u} \mathbb{M}$ is defined similarly: here we require that $\dot{\gamma}(t) \in \operatorname{span}\left\{\widehat{X}_{1}^{u}(\gamma(t)), \ldots, \widehat{X}_{\operatorname{dim} H_{1}}^{u}(\gamma(t))\right\}=\widehat{H}_{\gamma(t)}^{u} \mathbb{M}$ for almost all $t \in[0,1]$ with respect to the Lebesgue measure on $[0,1]$.

Theorem 2.10 (see [2, 1] for smooth case and [KmVod, Km9] for $C^{1, \alpha}$-smooth case). Every two points of $\mathbb{M}$ can be joined by a horizontal curve. 
Definition 2.11. Given $x, y \in \mathbb{M}$, the Carnot-Carathéodory distance $d_{c c}(x, y)$ is defined as

$$
d_{c c}(x, y)=\inf \left\{\ell(\gamma): \gamma:[0,1] \rightarrow \mathbb{M}, \dot{\gamma}(t) \in H_{\gamma(t)} \mathbb{M}\right\}
$$

where the length $\ell$ of each (horizontal) curve $\gamma$ is calculated with respect to the Riemannian tensor on $\mathbb{M}$.

For $x, y \in \mathcal{G}^{u} \mathbb{M}$ the Carnot-Carathéodory distance $d_{c c}^{u}(x, y)$ is defined as

$$
d_{c c}^{u}(x, y)=\inf \left\{\ell^{u}(\gamma): \gamma:[0,1] \rightarrow \mathcal{G}^{u} \mathbb{M}, \dot{\gamma}(t) \in \widehat{H}_{\gamma(t)}^{u} \mathbb{M}\right\},
$$

where the length $\ell^{u}$ of each (horizontal) curve $\gamma$ is calculated with respect to the Riemannian tensor on $\mathcal{G}^{u} \mathbb{M}$.

Denote the ball of radius $r$ in $d_{c c}$ centered at $x$ by $B_{c c}(x, r)$. Denote the ball of radius $r$ in $d_{c c}^{u}$ centered at $x$ by $B_{c c}^{u}(x, r)$.

Assumption 2.12. Henceforth we assume that $\varphi: \mathbb{M} \rightarrow \widetilde{\mathbb{M}}$ is a mapping of Carnot manifolds $\mathbb{M}$ and $\widetilde{\mathbb{M}}$. We specify its smoothness below in Assumption 2.26 .

Notation 2.13. Hereinafter, we use the following notation. Namely, we denote:

- the topological dimension of $\mathbb{M}(\widetilde{\mathbb{M}})$ by $N(\widetilde{N})$;

- the Hausdorff dimension of $\mathbb{M}(\widetilde{\mathbb{M}})$ by $\nu(\tilde{\nu})$;

- the depth of $\mathbb{M}(\widetilde{\mathbb{M}})$ by $M(\widetilde{M})$.

In addition, we consider

- horizontal subbundles $H=H_{1} \subset T \mathbb{M}$ and $\widetilde{H}=\widetilde{H}_{1} \subset T \widetilde{\mathbb{M}}$ on $\mathbb{M}(\widetilde{\mathbb{M}})$;

- subspaces $H \subset H_{2} \subset \ldots \subset H_{M}=T \mathbb{M}\left(\widetilde{H} \subset \widetilde{H}_{2} \subset \ldots \subset \widetilde{H}_{M}=T \widetilde{\mathbb{M}}\right)$ of dimensions $n=n_{1}=\operatorname{dim} H_{1}<\operatorname{dim} H_{2}<\ldots<\operatorname{dim} H_{M}=N(\tilde{n}=$ $\left.\tilde{n}_{1}=\operatorname{dim} \widetilde{H}_{1}<\operatorname{dim} \widetilde{H}_{2}<\ldots<\operatorname{dim} \widetilde{H}_{\widetilde{M}}=\tilde{N}\right)$ respectively at every point $x \in \mathbb{M}(\tilde{x} \in \widetilde{\mathbb{M}})$ (see Definition 2.1).

We put $n_{1}=\operatorname{dim} H_{1}, \tilde{n}_{1}=\operatorname{dim} \widetilde{H}_{1}, n_{k}=\operatorname{dim} H_{k}-\operatorname{dim} H_{k-1}\left(\tilde{n}_{k}=\right.$ $\left.\operatorname{dim} \widetilde{H}_{k}-\operatorname{dim} \widetilde{H}_{k-1}\right), k=2, \ldots, M(\widetilde{M})$.

Denote by $d_{c c}\left(\widetilde{d}_{c c}\right)$ the Carnot-Carathéodory metric in $\mathbb{M}(\widetilde{\mathbb{M}})$, and by $d_{c c}^{u}\left(\widetilde{d}_{c c}^{w}\right)$ the Carnot-Carathéodory metric in $\mathcal{G}^{u} \mathbb{M}\left(\mathcal{G}^{w} \widetilde{\mathbb{M}}\right)$.

Theorem 2.14 ([Vod4]). Suppose that $E \subset \mathbb{M}$ is an open set, and let $\varphi$ : $\mathbb{M} \rightarrow \widetilde{\mathbb{M}}$ be a mapping with $C^{1}$-smooth horizontal derivatives $X_{i} \varphi$ such that $X_{i} \varphi \in \widetilde{H}, i=1, \ldots, n$. Then, it is hc-differentiable in points of $E$. Namely, for a point $u \in E$, there exists a horizontal homomorphism $L_{u}:\left(\mathcal{G}^{u} \mathbb{M}, d_{c c}^{u}\right) \rightarrow$ $\left(\mathcal{G}^{\varphi(u)} \widetilde{\mathbb{M}}, \widetilde{d}_{c c}^{\varphi(u)}\right)$ of local Carnot groups such that it is continuous in $u$ and

$$
\widetilde{d}_{c c}\left(\varphi(w), L_{u}[w]\right)=o\left(d_{c c}(u, w)\right) \text { as } E \cap \mathcal{G}^{u} \mathbb{M} \ni w \rightarrow u
$$

where $o(\cdot)$ is locally uniform. 
Remark 2.15. Using the exponential mapping $\theta_{u}$, we can consider $L_{u}$ both as a homomorphism of local Carnot groups and as a homomorphism of Lie algebras of these local Carnot groups.

Notation 2.16. Henceforth we denote the $h c$-differential $L_{u}$ of $\varphi$ at $u$ by the symbol $\widehat{D} \varphi(u)$.

Corollary 2.17 ([Vod4] $)$. Let $\varphi: \mathbb{M} \rightarrow \widetilde{\mathbb{M}}$ be a contact $(i$. e., $D \varphi\langle H\rangle \subset \widetilde{H}$ ) $C^{1}$-mapping of Carnot manifolds (in the Riemannian sense). Then, it is continuously hc-differentiable everywhere on $\mathbb{M}$.

Property 2.18. Suppose that $\varphi \in C^{1}(\mathbb{M}, \widetilde{M})$. Then, the matrices of its differential $D \varphi(u)$ (in the bases $\left\{X_{i}(u)\right\}_{i=1}^{N}$ and $\left.\left\{\widetilde{X}_{i}(\varphi(u))\right\}_{i=1}^{\widetilde{N}}\right)$ and of its $h c$-differential $\widehat{D} \varphi(u)$ (in the bases $\left\{\widehat{X}_{i}^{u}(v)\right\}_{i=1}^{N}$ and $\left\{\widehat{X}_{i}(\varphi(v))\right\}_{i=1}^{\widetilde{N}}$, where $v$ need not be equal to $u$ ) have the following structures:

$$
\begin{aligned}
& D \varphi(u)=\left(\begin{array}{ccccccccc}
\mathcal{V}_{1}(u) & * & * & * & * & * & * & \ldots & * \\
0 & \mathcal{V}_{2}(u) & * & * & * & * & * & \ldots & * \\
0 & 0 & \mathcal{V}_{3}(u) & * & * & * & * & \ldots & * \\
\vdots & \vdots & \vdots & \ddots & \ddots & \vdots & * & \ldots & * \\
0 & \ldots & 0 & \vdots & \mathcal{V}_{\widetilde{M}-1}(u) & * & * & \ldots & * \\
0 & \ldots & 0 & \ldots & 0 & \mathcal{V}_{\widetilde{M}}(u) & * & \ldots & *
\end{array}\right) \\
& \widehat{D} \varphi(u)=\left(\begin{array}{ccccccccc}
\mathcal{V}_{1}(u) & 0 & & \ldots & 0 & 0 & 0 & \ldots & 0 \\
0 & \mathcal{V}_{2}(u) & 0 & \ldots & \vdots & 0 & 0 & \ldots & 0 \\
0 & 0 & \mathcal{V}_{3}(u) & 0 & 0 & 0 & 0 & \ldots & 0 \\
\vdots & \vdots & \vdots & \ddots & \ddots & \vdots & 0 & \ldots & 0 \\
0 & \ldots & 0 & \vdots & \mathcal{V}_{\widetilde{M}-1}(u) & 0 & 0 & \ldots & 0 \\
0 & \ldots & 0 & \ldots & 0 & \mathcal{V}_{\widetilde{M}}(u) & 0 & \ldots & 0
\end{array}\right),
\end{aligned}
$$

where each block $\mathcal{V}_{i}$ is of size $\left(n_{i} \times \tilde{n}_{i}\right), i=1, \ldots, M$. Note that, the blocks $\mathcal{V}_{i}$ are the same in the matrices of $D \varphi(u)$ and $\widehat{D} \varphi(u)$.

Assumption 2.19. Throughout the article we assume that:

- $N \geq \tilde{N}$;

- If the matrix of $\widehat{D} \varphi(x)$ has full rank at at least one point then we suppose that $n \geq \tilde{n}$. Otherwise, we suppose that $n_{i} \geq \tilde{n}_{i}, i=1, \ldots, M$ (see Proposition 2.21). 
Notation 2.20. Given $\varphi \in C^{1}(\mathbb{M}, \widetilde{\mathbb{M}})$, denote by $Z$ the set of points $x \in \mathbb{M}$ with $\operatorname{rank}(D \varphi(x))<\widetilde{N}$.

Proposition 2.21. I. Given $\varphi \in C^{1}(\mathbb{M}, \widetilde{\mathbb{M}})$, consider a point $x \in \mathbb{M} \backslash Z$.

(a) If $\widehat{D} \varphi(x)\left(V_{1}\right)=\widetilde{V}_{1}$ then $\widehat{D} \varphi(x)\left(V_{i}\right)=\widetilde{V}_{i}$ for all $i=1, \ldots, M$, and $\operatorname{rank} \widehat{D} \varphi(x)=\widetilde{N}$.

(b) If $\widehat{D} \varphi(x)\left(V_{1}\right) \varsubsetneqq \widetilde{V}_{1}$ then $\operatorname{rank} \widehat{D} \varphi(x)<\widetilde{N}$.

II. At the points $x \in \mathbb{M} \backslash Z$ with $\operatorname{rank} \widehat{D} \varphi(x)=\widetilde{N}$ we have

(a) $D \varphi(x)\left(H_{i}\right)=\widetilde{H}_{i}, i=1, \ldots, \widetilde{M}$;

(b) $\mathcal{D} \varphi(x)\left(H_{i} / H_{i-1}\right)=\widetilde{H}_{i} / \widetilde{H}_{i-1}, i=2, \ldots, \widetilde{M}$. Here for every element $\mathcal{Y} \in H_{i} / H_{i-1}$ there exists $Y \in H_{i}$ such that

$$
\mathcal{Y}=\left\{Y+T: T \in H_{i-1}\right\}=Y+H_{i-1},
$$

and we define for $\mathcal{Y}$ the value $\mathcal{D} \varphi\langle\mathcal{Y}\rangle$ as the class

$$
\begin{aligned}
\mathcal{D} \varphi\langle\mathcal{Y}\rangle=\left\{D \varphi\langle Y\rangle+D \varphi\langle T\rangle: Y \in H_{i} \text { is fixed, } T\right. & \left.\in H_{i-1}\right\} \\
& =D \varphi\langle Y\rangle+D \varphi\left\langle H_{i-1}\right\rangle .
\end{aligned}
$$

Proof. I. (a) Fix $x \in \mathbb{M} \backslash Z$ such that $\widehat{D} \varphi(x)\left(V_{1}\right)=\widetilde{V}_{1}$ and denote $\widehat{D} \varphi(x)$ by $L$. Verify that $L V_{2}=\widetilde{V}_{2}$. By taking into account property (3) of Definition 2.1 and the property $L[X, Y]=[L X, L Y]$ of a group homomorphism $L$, we infer that $L\left(V_{2}\right) \subset \widetilde{V}_{2}$. Verify that $\widetilde{V}_{2} \subset L\left(V_{2}\right)$. By property (4) of Definition 2.1 , for each element $\widetilde{Y} \in \widetilde{V}_{2}$ we have $\widetilde{Y}=\left[\widetilde{Y}_{1}, \widetilde{Y}_{2}\right]$, where $\widetilde{Y}_{1}, \widetilde{Y}_{2} \in$ $\widetilde{V}_{1}$. Since $\widetilde{Y}_{i}=L Y_{i}$, where $Y_{i} \in V_{1}, i=1,2$, it follows that

$$
\widetilde{Y}=\left[\widetilde{Y}_{1}, \widetilde{Y}_{2}\right]=\left[L Y_{1}, L Y_{2}\right]=L\left[Y_{1}, Y_{2}\right]=L Y,
$$

where $Y \in V_{2}$. Thus, $\widehat{D} \varphi(x)\left(V_{2}\right)=\widetilde{V}_{2}$.

Similar arguments show that $\widehat{D} \varphi(x)\left(V_{i}\right)=\widetilde{V}_{i}$ for all $i=3, \ldots, M$. Thus, $\widehat{D} \varphi(x)\left[\mathbb{G}_{x} \mathbb{M}\right]=\mathbb{G}_{\varphi(x)} \widetilde{\mathbb{M}}$, and $\operatorname{rank} \widehat{D} \varphi(x)=\widetilde{N}$.

(b) Note that the image of every basis vector field under the $h c$-differential $\widehat{D} \varphi(x)$ is a vector field of the same degree or the zero vector field (i. e., the $h c$-differential cannot decrease the degree of each basis vector field). If $\widehat{D} \varphi(x)\left(V_{1}\right) \varsubsetneqq \widetilde{V}_{1}$ then $\widehat{D} \varphi(x)^{-1}\left(\widetilde{V}_{1} \backslash\left[\widehat{D} \varphi(x)\left(V_{1}\right)\right]\right)=\varnothing$, and $\widehat{D} \varphi(x)\left[\mathbb{G}_{x} \mathbb{M}\right] \neq$ $\mathbb{G}_{\varphi(x)} \widetilde{\mathrm{M}}$. Consequently, $\operatorname{rank} \widehat{D} \varphi(x)<\widetilde{N}$.

We prove claims (a) and (b) simultaneously. The properties of the Riemannian differential $D \varphi(x)$ (see (2.4) ) imply that $D \varphi(x)\left(H_{k}\right) \subset \widetilde{H}_{k}$ for $k=1, \ldots, M$. In particular, $D \varphi(x)\left(H_{1}\right) \subset \widetilde{H}_{1}$ by the definition of a contact mapping. Moreover, $D \varphi(x)\left(H_{1}\right)=\widetilde{H}_{1}$ if and only if $\widehat{D} \varphi(x)\left(V_{1}\right)=\widetilde{V}_{1}$. 
In order to verify that $\mathcal{D} \varphi(x)\left(H_{2} / H_{1}\right) \subset \widetilde{H}_{2} / \widetilde{H}_{1}$, take $\mathcal{Y} \in H_{2} / H_{1}$. Consider $\mathcal{Y}$ as a class of sums of a fixed element $Y$ of $H_{2}$, which may have nonzero coordinates (in the basis $\left\{X_{i}\right\}_{i=1}^{N}$ ) only with indices greater than $n_{1}$ and less than $n_{1}+n_{2}+1$, and all elements of $H_{1}$ :

$$
\mathcal{Y}=\left\{Y+T: Y \in H_{2} \text { is fixed, } T \in H_{1}\right\} .
$$

Then, the structure of $D \varphi(x)$ implies that the image

$$
\widetilde{\mathcal{Y}}=\mathcal{D} \varphi(x) \mathcal{Y}=D \varphi(x) Y+D \varphi(x)(H)=D \varphi(x) Y+\widetilde{H} \in \widetilde{H}_{2} / \widetilde{H}_{1}
$$

consists of the vectors with only the first $\tilde{n}_{1}+\tilde{n}_{2}$ nonzero components. Moreover, the components with indexes from $\tilde{n}_{1}+1$ to $\tilde{n}_{2}$ are the same for all vectors from $\widetilde{\mathcal{Y}}$. (To verify that, it suffices to write the vectors $Y$ and $T$ in the basis $\left\{X_{i}\right\}_{i=1}^{N}$, and the matrix of $D \varphi$ in the bases $\left\{X_{i}\right\}_{i=1}^{N}$ and $\left\{\widetilde{X}_{i}\right\}_{i=1}^{\widetilde{N}}$, and consider the product of this matrix and these vectors.) Thus, $\mathcal{D} \varphi(x)\left(H_{2} / H_{1}\right) \subset \widetilde{H}_{2} / \widetilde{H}_{1}$.

Verify that $\mathcal{D} \varphi(x)\left(H_{2} / H_{1}\right) \supset \widetilde{H}_{2} / \widetilde{H}_{1}$. Take $\widetilde{\mathcal{Y}} \in \widetilde{H}_{2} / \widetilde{H}_{1}$ and assume on the contrary that $\widetilde{\mathcal{Y}} \notin \mathcal{D} \varphi(x)\left(H_{2} / H_{1}\right)$. Consider a vector field $\widetilde{Y} \in \widetilde{\mathcal{Y}}$; then, $\widetilde{Y}=\widetilde{Y}_{2}+\widetilde{Y}_{1}$ with $\widetilde{Y}_{1} \in \widetilde{H}_{1}$. Without loss of generality we may assume that $\widetilde{Y}_{1}=0$. Since $\operatorname{rank} \widehat{D} \varphi(x)=\widetilde{N}$, it follows that the images $D \varphi(x)\left(T_{x} \mathbb{M}\right)$ and $(D \varphi)_{\widetilde{M}}(x)\left(T_{x} \mathbb{M}\right)$ coincide, as in both cases we obtain the whole $T_{\varphi(x)} \widetilde{\mathbb{M}}$. Here the linear operator $(D \varphi)_{\widetilde{M}}(x)$ acts on $T_{x} \mathbb{M}$ (instead of $\left.\mathbb{G}_{x} \mathbb{M}\right)$, and its matrix in the bases $\left\{X_{i}(x)\right\}_{i=1}^{N}$ and $\left\{\widetilde{X}_{i}(\varphi(x))\right\}_{i=1}^{\widetilde{N}}$ equals that of $\widehat{D} \varphi(x)$ (written in the bases $\left\{\widehat{X}_{i}^{x}(x)\right\}_{i=1}^{N}$ and $\left.\left\{\widehat{\widetilde{X}}_{i}^{\varphi(x)}(\varphi(x))\right\}_{i=1}^{\widetilde{N}}\right)$. Denote by $L$ the matrix of this operator. Then, there exists a vector $Y_{0}$ with $L Y_{0}=\tilde{Y}$. Without loss of generality we may assume that $Y_{0}$ has at most $n_{2}$ nonzero components with indices from $n_{1}+1$ to $n_{1}+n_{2}$. Consequently,

$$
\widetilde{Y}=L Y_{0}=D \varphi(x) Y_{0}+[L-D \varphi(x)] Y_{0},
$$

where $D \varphi(x) Y_{0} \in \widetilde{H}_{2}$ and $[L-D \varphi(x)] Y_{0} \in \widetilde{H}_{1}$. Since $D \varphi(x)\left(H_{1}\right)=\widetilde{H}_{1}$, there exists $Y_{1} \in D \varphi(x)^{-1}\left([L-D \varphi(x)] Y_{0}\right) \cap H_{1}$. Put $Y=Y_{0}+Y_{1}$, and then $D \varphi(x) Y=\widetilde{Y}$ and $Y \in H_{2}$. Thus, $\mathcal{D} \varphi(x)\left(H_{2} / H_{1}\right)=\widetilde{H}_{2} / \widetilde{H}_{1}$ and, moreover, $D \varphi(x)\left(H_{2}\right)=\widetilde{H}_{2}$.

Similarly, we can show that $\mathcal{D} \varphi(x)\left(H_{i} / H_{i-1}\right)=\widetilde{H}_{i} / \widetilde{H}_{i-1}$ and $D \varphi(x)\left(H_{i}\right)=$ $\widetilde{H}_{i}, i=3, \ldots, \widetilde{M}$. The proposition follows.

\section{Claim I implies}

Corollary 2.22. The existence of $x \in \mathbb{M}$ with $\operatorname{rank} \widehat{D} \varphi(x)=\widetilde{N}$ implies that $n_{i} \geq \tilde{n}_{i}, i=1, \ldots, M$. 
Definition 2.23. The set

$$
\chi=\{x \in \mathbb{M} \backslash Z: \operatorname{rank} \widehat{D} \varphi(x)<\tilde{N}\}
$$

is called the characteristic set. The points of $\chi$ are called characteristic points.

For $t \in \widetilde{\mathbb{M}}$, denote the intersection $\varphi^{-1}(t) \cap \chi$ by $\chi_{t}$.

Remark 2.24. For $\widetilde{\mathbb{M}}=\mathbb{R}$ this definition coincides with the definition of the characteristic set given by P. Pansu [Pa] and J. Heinonen [He]: in this case the condition rank $\widehat{D} \varphi(x)<1$ implies that all the horizontal derivatives $X_{i} \varphi(x), i=1, \ldots, n$, vanish at $x$, and vice versa.

Remark 2.25. Proposition 2.21 explains the second part of Assumption 2.19, if $\mathbb{M} \neq \chi$ then we do not need to assume that $n_{i} \geq \tilde{n}_{i}$ for $i \geq 2$.

Assumption 2.26. If $\mathcal{H}^{\nu}(\chi)=\mathcal{H}^{N}(\chi)=0$ then we assume that $\varphi \in$ $C^{1}(\mathbb{M}, \widetilde{\mathbb{M}})$ and $X_{i} \in C^{2}(\mathbb{M}), i=1, \ldots, N$ (this condition is sufficient for establishing the $h c$-differentiability of $\varphi$ ); otherwise, we assume that $\varphi \in$ $C^{M+1}(\mathbb{M}, \widetilde{\mathbb{M}})$ and $X_{i} \in C^{M+1}(\mathbb{M}), i=1, \ldots, N$.

Definition 2.27. The set

$$
\mathbb{D}=\{x \in \mathbb{M}: \operatorname{rank} \widehat{D} \varphi(x)=\widetilde{N}\}
$$

is called the regular set. If $x \in \mathbb{D}$ then we say that $x$ is a regular point.

Lemma 2.28. I. For the set

$$
\begin{aligned}
& \zeta=\left\{x \in \mathbb{M} \backslash Z: \exists\left\{X_{i_{1}}, \ldots, X_{i_{\tilde{N}}}\right\}\right. \\
&\left.\left(\operatorname{rank}\left(\left[X_{i_{j}} \varphi\right](x)\right)_{j=1}^{\widetilde{N}}=\widetilde{N}\right) \Rightarrow\left(\sum_{j=1}^{\widetilde{N}} \operatorname{deg} X_{i_{j}}<\tilde{\nu}\right)\right\}
\end{aligned}
$$

we have $\zeta=\varnothing$ (see Notation 2.20 for the description of $Z$ ).

II. If there exists a family $\left\{X_{i_{1}}, \ldots, X_{i_{\widetilde{N}}}\right\}$ of vector fields with the properties $\operatorname{rank}\left(\left[X_{i_{j}} \varphi\right](x)\right)=\widetilde{N}$ and $\sum_{j=1}^{\widetilde{N}} \operatorname{deg} X_{i_{j}}=\tilde{\nu}$, then we have $\operatorname{deg} X_{i_{j}} \leq \widetilde{M}$, $j=1, \ldots, \widetilde{N}$.

Proof. Fix $x \in \mathbb{M}$ and consider the matrix of $D \varphi(x)$ :

$$
D \varphi(x)=\left(\begin{array}{ccccccccc}
\mathcal{V}_{1}(u) & * & * & * & * & * & * & \ldots & * \\
0 & \mathcal{V}_{2}(u) & * & * & * & * & * & \ldots & * \\
0 & 0 & \mathcal{V}_{3}(u) & * & * & * & * & \ldots & * \\
\vdots & \vdots & \vdots & \ddots & \ddots & \vdots & * & \ldots & * \\
0 & \ldots & 0 & \vdots & \mathcal{V}_{\widetilde{M}-1}(u) & * & * & \ldots & * \\
0 & \ldots & 0 & \ldots & 0 & \mathcal{V}_{\widetilde{M}}(u) & * & \ldots & *
\end{array}\right)
$$


Choose $\widetilde{N}$ linearly independent columns with the minimal possible sum of the corresponding degrees (we consider $\operatorname{deg} X_{j}$ as the degree of column $j$, $j=1, \ldots, N)$.

To this end, we must choose the maximal possible quantity of vectors from the blocks corresponding to the minimal degrees. In the first block, we can choose at most $\tilde{n}_{1}$ linearly independent vectors. Next, take columns from $n_{1}+1$ to $n_{1}+n_{2}$ and the corresponding "diagonal" block. In this block, we can choose at most $\tilde{n}_{2}$ linearly independent $\tilde{n}_{2}$-dimensional elements.

On assuming that there are more than $\tilde{n}_{2}$ linearly independent columns, we obtain a contradiction. Indeed, since the "diagonal" block is an $\left(\tilde{n}_{2} \times n_{2}\right)$ matrix, there exists an elementary transformation reducing at least $n_{2}-\tilde{n}_{2}$ of its columns to zero. Apply this transformation to the columns of the matrix of $D \varphi(x)$. Then, this block of size $\left(\tilde{n}_{1}+\tilde{n}_{2}\right) \times\left(n_{1}+n_{2}\right)$ includes $n_{1}+n_{2}-\tilde{n}_{2}$ columns of dimension $\tilde{n}_{1}$ (more exactly, these column vectors belong to $\mathbb{R}^{\tilde{n}_{1}} \times 0^{\tilde{n}_{2}}$ ). Recall that the maximal number of linearly independent columns is $\tilde{n}_{1}$, and we have already chosen them in the first "diagonal" block. Suppose that there are less than $\tilde{n}_{1}+\tilde{n}_{2}$ linearly independent columns. Then, since $\operatorname{rank} D \varphi(x)=\widetilde{N}$, a "missing" column can be "replaced" by a column of a higher degree. Thus, we can obtain the minimal possible sum of degrees if we have only $n_{1}-\tilde{n}_{1}+n_{2}-\tilde{n}_{2}$ linearly dependent among the first $n_{1}+n_{2}$ columns. Therefore, the corresponding sum of degrees equals $\tilde{n}_{1}+2 \tilde{n}_{2}$.

Applying further the same arguments to the degrees $3, \ldots, \widetilde{M}$, we conclude that the minimal possible sum of degrees of linearly independent vector fields $\left\{X_{i_{j}} \varphi\right\}_{j=1}^{\widetilde{N}}$ is equal to $\tilde{\nu}$. Thus, claim $\mathbf{I}$ is proved.

Claim II follows since, firstly, we can obtain the sum equal to $\tilde{\nu}$ only by considering the first $\widetilde{M}$ blocks, and, secondly, if we have less than $\tilde{n}_{k}$ linearly independent vector fields on step $k \leq \widetilde{M}$ then the sum of degrees corresponding to the resulting collection is strictly greater than $\tilde{\nu}$.

Theorem 2.29. I. The characteristic set $\chi$ coincides with

$$
\begin{aligned}
&\left\{x \in \mathbb{M} \backslash Z: \forall\left\{X_{i_{1}}, \ldots, X_{i_{\tilde{N}}}\right\}\right. \\
&\left.\left(\operatorname{rank}\left(\left[X_{i_{j}} \varphi\right](x)\right)_{j=1}^{\tilde{N}}=\tilde{N}\right) \Rightarrow\left(\sum_{j=1}^{\widetilde{N}} \operatorname{deg} X_{i_{j}}>\tilde{\nu}\right)\right\} .
\end{aligned}
$$

II. The regular set $\mathbb{D}$ coincides with

$$
\begin{aligned}
\{x \in \mathbb{M} \backslash Z: & \exists\left\{X_{i_{1}}, \ldots, X_{i_{\tilde{N}}}\right\} \\
& \left.\left(\operatorname{rank}\left(\left[X_{i_{j}} \varphi\right](x)\right)_{j=1}^{\widetilde{N}}=\widetilde{N}\right) \Rightarrow\left(\sum_{j=1}^{\widetilde{N}} \operatorname{deg} X_{i_{j}}=\tilde{\nu}\right)\right\} .
\end{aligned}
$$


Proof. I. (a) Denote the set in (2.6) by $A$, and verify that $A \subset \chi$. Consider a point $x \in A$. By (2.6), every $\widetilde{N}$ columns of the matrix of $D \varphi(x)$ with indices $i_{1}, \ldots, i_{\widetilde{N}}$ corresponding to a collection $\left\{X_{i_{1}}, \ldots, X_{i_{\widetilde{N}}}\right\}$ with $\sum_{j=1}^{\widetilde{N}} \operatorname{deg} X_{i_{j}}=\tilde{\nu}$ are linearly dependent. Assume on the contrary that the rank of the matrix of $\widehat{D} \varphi(x)$ equals $\widetilde{N}$, and consequently, there exist $\widetilde{N}$ linearly independent columns in the matrix of $\widehat{D} \varphi(x)$. The matrix of $\widehat{D} \varphi(x)$ has a block structure, where the block $k$ is an $\left(\tilde{n}_{k} \times n_{k}\right)$-matrix. Thus, in each block, only $\tilde{n}_{k}$ columns can be linearly independent. Consequently, the sum of the degrees of the vector fields corresponding to these linearly independent columns equals $\tilde{\nu}$.

The relation between the matrices of $D \varphi(x)$ and $\widehat{D} \varphi(x)$ implies that the corresponding columns of the matrix of $D \varphi(x)$ are also linearly independent, and the sum of the degrees of the corresponding vector fields is equal to $\tilde{\nu}$. Thus, we arrive at a contradiction.

The argument above implies that $A \subset \chi$.

(b) Verify that $\chi \subset A$. Consider $x \in \chi$. Since $\operatorname{rank} \widehat{D} \varphi(x)<\widetilde{N}$, it follows that every $\widetilde{N}$ columns are linearly dependent. Our goal is to show that if we take the columns of the matrix of $D \varphi(x)$ with indices $i_{1}, \ldots, i_{\widetilde{N}}$ such that

$$
\sum_{j=1}^{\tilde{N}} \operatorname{deg} X_{i_{j}} \leq \tilde{\nu}
$$

then

$$
\operatorname{rank}\left(\left[X_{i_{j}} \varphi\right](x)\right)_{j=1}^{\widetilde{N}}<\tilde{N} .
$$

In view of claim I of Lemma 2.28, it suffices to consider the columns with indices $i_{1}, \ldots, i_{\widetilde{N}}$ satisfying

$$
\sum_{j=1}^{\tilde{N}} \operatorname{deg} X_{i_{j}}=\tilde{\nu}
$$

Take $\widetilde{N}$ columns $c_{i_{1}}, \ldots, c_{i_{\tilde{N}}}$ of the matrix of $\widehat{D} \varphi(x)$ corresponding to some vectors $X_{i_{1}}, \ldots, X_{i_{\tilde{N}}}$ with $\sum_{j=1}^{\widetilde{N}} \operatorname{deg} X_{i_{j}}=\tilde{\nu}$. Since

$$
\operatorname{rank}\left(\left\{c_{i_{1}}, \ldots, c_{i_{\tilde{N}}}\right\}\right)<\tilde{N}
$$

there exists a transformation $\tau$ of the matrix of $\widehat{D} \varphi(x)$ taking at least one of $c_{i_{j}}$ to a zero column, and preserving the block structure of the initial matrix. Denote this column by $c_{i_{j_{0}}}$. Here we assume that $j_{0}$ is the minimal 
number with this property. Put $k=\operatorname{deg} X_{i_{j_{0}}}-1$. Because of the block structure of the matrix $\widehat{D} \varphi(x)$, the numbers of nonzero entries of $c_{i_{j_{0}}}$ are at least $\operatorname{dim} \widetilde{H}_{k}+1$, and at most $\operatorname{dim} \widetilde{H}_{k+1}$. Without loss of generality we may assume that $i_{j_{0}}=\operatorname{dim} H_{k}+1$. Since we cannot transform all preceding columns into zero columns, it follows that $j_{0}=\operatorname{dim} \widetilde{H}_{k}+1$.

Apply the same transformation $\tau$ to the matrix of $D \varphi(x)$, and consider the images of $j_{0}$ columns with indices $i_{1}, \ldots, i_{j_{0}}$. Note that we may regard the image of column $i_{j_{0}}=\operatorname{dim} H_{k}+1$ as an element of $\mathbb{R}^{\operatorname{dim} \widetilde{H}_{k}}$. Taking the structure of $D \varphi(x)$ into account, we have $j_{0}=\operatorname{dim} \widetilde{H}_{k}+1$ vectors (columns) belonging to the space $\mathbb{R}^{\operatorname{dim} \widetilde{H}_{k}}$ (because the components with indices greater than $\operatorname{dim} \widetilde{H}_{k}$ vanish). Thus, the rank of this collection equals $\operatorname{dim} \widetilde{H}_{k}<j_{0}$. Consequently, the rank of $\left\{X_{i_{1}} \varphi, \ldots, X_{i_{\tilde{N}}} \varphi\right\}$ is strictly less than $\widetilde{N}$. Since the collections of $\widetilde{N}$ vectors with the sum of degrees equal to $\tilde{\nu}$ appear only in the matrix of $\widehat{D} \varphi(x)$ (see Lemma 2.28), we see that if the sum of the degrees is equal to $\tilde{\nu}$, then the rank is strictly less than $\tilde{N}$. By taking Lemma 2.28 and the fact that $\operatorname{rank}(D \varphi(x))=\widetilde{N}$ into account, we see that $\chi \subset A$.

II. (a) Denote the set in (2.7) by $B$. Assume the contrary and take $x \in B$ with $\operatorname{rank}(\widehat{D} \varphi(x))<\widetilde{N}$. Step I yields $x \in A$, and thus, we obtain a contradiction since $A \cap B=\varnothing$. Consequently, $B \subset \mathbb{D}$.

(b) Take $x \in \mathbb{D}$. Since $\operatorname{rank} \widehat{D} \varphi(x)=\widetilde{N}$, it follows that $\operatorname{rank} D \varphi(x)=\widetilde{N}$ and $x \notin Z$. Take an arbitrary collection of $\widetilde{N}$ linearly independent columns of the matrix of $\widehat{D} \varphi(x)$. Consequently, the corresponding columns of the matrix of $D \varphi(x)$ are linearly independent as well, and $x \in B$. Thus, $\mathbb{D} \subset B$.

The theorem follows.

\section{$3 \quad$ Properties of Level Sets}

In this section, we assume that $x \in \mathbb{M} \backslash Z$.

First of all, we introduce a new metric, which is equivalent to the initial one and simplifies our computations.

Definition 3.1. Let $\mathbb{M}$ be a Carnot manifold of topological dimension $N$ and depth $M$, and put $x=\exp \left(\sum_{i=1}^{N} x_{i} X_{i}\right)(g)$. Define the distance $d_{2}(x, g)$ as 
follows:

$$
\begin{aligned}
d_{2}(x, g)=\max \{ & \left(\sum_{j=1}^{n_{1}}\left|x_{j}\right|^{2}\right)^{\frac{1}{2}}, \\
& \left.\left(\sum_{j=n_{1}+1}^{n_{1}+n_{2}}\left|x_{j}\right|^{2}\right)^{\frac{1}{2 \cdot \operatorname{deg} X_{n_{1}+1}}}, \ldots,\left(\sum_{j=N-n_{M}+1}^{N}\left|x_{j}\right|^{2}\right)^{\frac{1}{2 \cdot \operatorname{deg} X_{N}}}\right\} .
\end{aligned}
$$

A similar metric $d_{2}^{u}$ is introduced on the local Carnot group $\mathcal{G}^{u} \mathbb{M}$.

The set $\left\{y \in \mathbb{M}: d_{2}(y, x)<r\right\}$ is called the ball of radius $r>0$ centered at $x$ and denoted by $\operatorname{Box}_{2}(x, r)$. Similarly, $\operatorname{Box}_{2}^{u}(x, r)$ stands for the ball in $d_{2}^{u}$ of radius $r>0$ centered at $x$.

Remark 3.2. The preimage of $\operatorname{Box}_{2}(x, r)$ in the metric $d_{2}$ under the mapping $\theta_{x}$ equals

$$
\operatorname{Box}_{2}(0, r)=B_{2}^{n_{1}}(x, r) \times B_{2}^{n_{2}}\left(x, r^{2}\right) \times \ldots \times B_{2}^{n_{M}}\left(x, r^{M}\right),
$$

where $B_{2}^{n_{i}}$ is a Euclidean ball of dimension $n_{i}, i=1, \ldots, M$.

Observe that $\operatorname{Box}_{2}^{u}(u, r)=\operatorname{Box}_{2}(u, r)$ in the quasimetric $d_{2}^{u}$ since

$$
\exp \left(\sum_{i=1}^{N} x_{i} \widehat{X}_{i}^{u}\right)(u)=\exp \left(\sum_{i=1}^{N} x_{i} X_{i}\right)(u)
$$

for all collections $\left\{x_{i}\right\}_{i=1}^{N}$ such that both parts of (3.1) make sense [Vod4].

The following proposition is useful for proving the main results.

Proposition 3.3. Let $\mathbb{M}$ be a Carnot manifold of topological dimension $N$ and depth $M$. Given a sufficiently small compact domain $U \Subset \mathbb{M}$, there exist positive constants $C>0$ and $r_{0}>0$ depending on $U, M$, and $N$ such that all points $u$ and $v$ of $U$ satisfy

$$
\bigcup_{x \in \operatorname{Box}_{2}^{u}(v, r)} \operatorname{Box}_{2}^{u}(x, \xi) \subseteq \operatorname{Box}_{2}^{u}(v, r+C \xi), \quad 0<\xi, r \leq r_{0} .
$$

Proof. The proof follows the scheme of proof of the similar lemma for boxes in the metric $d_{\infty}^{u}$ of [VodKm1, KmVod].

Put $x=\exp \left(\sum_{i=1}^{N} x_{i} \widehat{X}_{i}^{u}\right)(v), d_{2}^{u}(v, x) \leq r$, and $z=\exp \left(\sum_{i=1}^{N} z_{i} \widehat{X}_{i}^{u}\right)(x)$, $d_{2}^{u}(x, z) \leq \xi$. Estimate the distance $d_{2}^{u}(v, z)$ applying group operation to points $x$ and $z$. Namely, estimate the coefficients $\left\{\zeta_{i}\right\}_{i=1}^{N}$ satisfying $z=$ $\exp \left(\sum_{i=1}^{N} \zeta_{i} \widehat{X}_{i}^{u}\right)(v)$ 
Case of $\operatorname{deg} X_{i}=1$. We have

$$
\sum_{i=1}^{n}\left(\zeta_{i}\right)^{2}=\sum_{i=1}^{n}\left(x_{i}+z_{i}\right)^{2}=\sum_{i=1}^{n}\left(x_{i}\right)^{2}+\sum_{i=1}^{n}\left(z_{i}\right)^{2}+2 \sum_{i=1}^{n}\left(x_{i} z_{i}\right) \leq(r+\xi)^{2 \operatorname{deg} X_{i}} .
$$

Case of $\operatorname{deg} X_{i}=2$. We have

$$
\begin{gathered}
\sum_{i=n+1}^{n+n_{2}}\left(\zeta_{i}\right)^{2} \leq \sum_{i=n+1}^{n+n_{2}}\left(x_{i}+z_{i}+\sum_{\substack{\left|e_{l}+e_{j}\right|_{h}=2, l<j}} \widehat{F}_{e_{l}, e_{j}}^{i}(u)\left(x_{l} z_{j}-z_{l} x_{j}\right)\right)^{2} \\
\leq r^{4}+\xi^{4}+2 r^{2} \xi^{2}+\sum_{i=n+1}^{n+n_{2}}\left(\sum_{\substack{\left|e_{l}+e_{j}\right|_{h}=2, l<j}} \widehat{F}_{e_{l}, e_{j}}^{i}(u)\left(x_{l} z_{j}-z_{l} x_{j}\right)\right)^{2} \\
+2 \sum_{i=n+1}^{n+n_{2}}\left(\left(x_{i}+z_{i}\right) \sum_{\substack{\left|e_{l}+e_{j}\right|_{h}=2, l<j}} \widehat{F}_{e_{l}, e_{j}}^{i}(u)\left(x_{l} z_{j}-z_{l} x_{j}\right)\right) \\
\leq r^{4}+\xi^{4}+c_{i}(u) r^{2} \xi^{2}+b_{i}(u) r \xi(r+\xi)^{2} \\
\leq\left(r+a_{2}(u) \xi\right)^{4}=\left(r+a_{2}(u) \xi\right)^{2 \operatorname{deg} X_{i}},
\end{gathered}
$$

where $b_{i}(u)$ and $c_{i}(u)$ are linear combinations of the functions $\left\{\widehat{F}_{e_{l}, e_{j}}^{i}(u)\right\}_{l, j}$ on assuming that $\left|x_{l} z_{j}-z_{l} x_{j}\right|=2 r \xi$ and $\left|x_{i}+z_{i}\right|=r+\xi$ for all $i, l, j$. They are continuous with respect to $u \in U$. We can represent each sum $r^{4}+\xi^{4}+c_{i}(u) r^{2} \xi^{2}+b_{i}(u) r \xi(r+\xi)^{2}$ as $\left(r+d_{i}(u) \xi\right)^{4}$, where $d_{i}(u)$ depends on $b_{i}(u)$ and $c_{i}(u)$. Put $a_{2}(u)=\max _{i: \operatorname{deg} X_{i}=2} d_{i}(u)$ and assume without loss of generality that $a_{2}(u) \geq 1$.

CASE OF $\operatorname{deg} X_{i}=k>2$. Denote the sum $n+\sum_{j=2}^{k} n_{j}$ by $S_{k}$. Then, as in the previous case of $\operatorname{deg} X_{i}=2$, we obtain

$$
\begin{aligned}
\sum_{i=S_{k-1}+1}^{S_{k}}\left(\zeta_{i}\right)^{2} \leq \sum_{i=S_{k-1}+1}^{S_{k}}\left(x_{i}+z_{i}+\right. & \left.\sum_{\substack{|\mu+\beta|_{h}=k, \mu>0, \beta>0\\
}}\left|\widehat{F}_{\mu, \beta}^{i}(u)\right| x^{\mu} \cdot z^{\beta}\right)^{2} \\
& \leq\left(r+a_{k}(u) \xi\right)^{2 k}=\left(r+a_{k}(u) \xi\right)^{2 \operatorname{deg} X_{i}} .
\end{aligned}
$$

Here we use the property

$$
\sum_{\substack{|\mu+\beta|_{h}=k, \mu>0, \beta>0}}\left|\widehat{F}_{\mu, \beta}^{i}(u)\right| x^{\mu} \cdot z^{\beta} \leq \sum_{\substack{|\mu+\beta|_{h}=k, \mu>0, \beta>0}} c_{\mu, \beta}^{i}(u) r^{|\mu|_{h}} \cdot \xi^{|\beta|_{h}}
$$


and define each function $a_{k}(u)$ in the similar way as $a_{2}(u)$ in the case $\operatorname{deg} X_{i}=$ 2. We also assume without loss of generality that $a_{k}(u), c_{\mu, \beta}^{i}(u) \geq 1$. Put $a(u)=\max _{i} a_{i}(u)$. The estimates above yield

$$
\begin{aligned}
d_{2}^{u}(v, x)= & \max \left\{\left(\sum_{j=1}^{n}\left|\zeta_{j}\right|^{2}\right)^{\frac{1}{2}},\right. \\
& \left.\left(\sum_{j=n+1}^{n+n_{2}}\left|\zeta_{j}\right|^{2}\right)^{\frac{1}{2 \cdot \operatorname{deg} X_{n+1}}}, \ldots,\left(\sum_{j=N-n_{M}+1}^{N}\left|\zeta_{j}\right|^{2}\right)^{\frac{1}{2 \cdot \operatorname{deg} X_{N}}}\right\} \\
& \leq \max _{i}\left\{\left(r+a_{i}(u) \xi\right)^{\frac{\operatorname{deg} X_{i}}{\operatorname{deg} X_{i}}}\right\} \leq r+a(u) \xi .
\end{aligned}
$$

Since all $a_{i}(u)$ are continuous with respect to $u$, we may choose sufficiently large $C<\infty$ with $a(u) \leq C$ for all $u$ belonging to the given compact domain $U \Subset \mathbb{M}$. The lemma follows.

To prove the main theorems, we need a convenient quasimetric equivalent to the Riemannian metric.

Definition 3.4. Given

$$
y=\exp \left(\sum_{i=1}^{N} y_{i} X_{i}\right)(x),
$$

put $\rho(y, x)=\max _{i=1, \ldots, N}\left\{\left|y_{i}\right|\right\}$.

Notation 3.5. In Theorems 3.7 and 3.11, we establish some local results for a fixed point $x$. From now on we use the auxiliary mapping $\psi=\varphi \circ \theta_{x}$.

Notation 3.6. Put

$$
\begin{aligned}
\nu_{0}(x)=\min \left\{\nu: \exists\left\{X_{i_{1}}, \ldots, X_{i_{\tilde{N}}}\right\}\right. & \\
& \left.\left(\operatorname{rank}\left(\left[X_{i_{j}} \varphi\right](x)\right)_{j=1}^{\widetilde{N}}=\widetilde{N}\right) \Rightarrow\left(\sum_{j=1}^{\widetilde{N}} \operatorname{deg} X_{i_{j}}=\nu\right)\right\} .
\end{aligned}
$$

It is clear that $\left.\nu_{0}\right|_{\chi}>\tilde{\nu}$ and $\left.\nu_{0}\right|_{\mathbb{D}}=\tilde{\nu}$.

Theorem 3.7. Fix $x \in \varphi^{-1}(t)$. Then, in a neighborhood in $\mathbb{R}^{N}$, the $\mathcal{H}^{N-\widetilde{N}_{-}}$ measure of $T_{0}\left[\psi^{-1}(t)\right] \cap \operatorname{Box}_{2}(0, r)$ (see Remark [3.2) is equal to

$$
C r^{\nu-\nu_{0}(x)}(1+o(1))
$$

where $C$ is independent of $r$, and $o(1) \rightarrow 0$ as $r \rightarrow 0$. 
Remark 3.8. We emphasize that the mapping $\psi=\varphi \circ \theta_{x}$ acts on a neighborhood of the origin in $\mathbb{R}^{N}$. Consequently, the tangent plane to the level set $\psi^{-1}(t)$ lies in $\mathbb{R}^{N}$, and the intersection $T_{0}\left[\psi^{-1}(t)\right] \cap \operatorname{Box}_{2}(0, r)$ is well-defined.

Proof of Theorem 3.7. We split the proof into 6 steps. On step I we choose a suitable basis $\left\{w_{j}\right\}_{j=1}^{N}$ for the tangent space $\mathcal{T}=T_{0}\left[\psi^{-1}(t)\right]$ to the level set. On step II we define two projections of the basis vectors in $T_{0}\left[\psi^{-1}(t)\right]$. In particular, the first projection $\pi$ assigns to each basis vector $w_{j}$ some vector $p_{j}$ of the same degree, and the second projection assigns a standard vector $\frac{\pi_{j}}{\left|\pi_{j}\right|}$ in $\left\{e_{1}, \ldots, e_{N}\right\}$ to each basis vector $w_{j}$ for $T_{0}\left[\psi^{-1}(t)\right]$. On step III we show that $\operatorname{rank}\left(\sigma_{i} \psi\right)_{i=1}^{\widetilde{N}}=\widetilde{N}$ (henceforth $\sigma_{i} \psi$ stands for the action of the vector $\sigma_{i}$ on $\left.\psi, i=1, \ldots, N\right)$, where

$$
\left\{\sigma_{1}, \ldots, \sigma_{\widetilde{N}}\right\}=\left\{e_{1}, \ldots, e_{N}\right\} \backslash\left\{\frac{\pi_{1}}{\left|\pi_{1}\right|}, \ldots, \frac{\pi_{N-\widetilde{N}}}{\left|\pi_{N-\widetilde{N}}\right|}\right\}
$$

and on step IV we prove that the sum of the degrees of $\sigma_{i}, i=1, \ldots, \tilde{N}$, coincides with $\nu_{0}(x)$. Consequently, the sum of the degrees of $\pi_{j}, j=$ $1, \ldots, N-\tilde{N}$, equals $\nu-\nu_{0}(x)$. Further, on step $\mathbf{V}$ we deduce that the Lebesgue measure of $\operatorname{Box}_{2}(0, r) \cap \operatorname{span}\left\{p_{1}, \ldots, p_{N-\tilde{N}}\right\}$ equals $C r^{\nu-\nu_{0}(x)}$, where $C$ is independent of $r$. Finally, on step VI we prove that the length of $\mathbb{R} w_{j} \cap \operatorname{Box}_{2}(0, r)$ equals $O\left(r^{k(j)}\right)$ for sufficiently small $r>0$, and applying this result we show that $\pi\left(\mathcal{T} \cap \operatorname{Box}_{2}(0, r)\right)$ coincides with the $o(r)$-neighborhood of $\mathcal{S} \cap \operatorname{Box}_{2}(0, r)$ in $\mathcal{S}$, where $\mathcal{S}=\operatorname{span}\left\{p_{1}, \ldots, p_{N-\widetilde{N}}\right\}$ and $o(r)$ is taken with respect to the metric $d_{2}$. The theorem follows from the last result.

Step I. Consider the normal coordinates at the point $x$. Recall that $\mathcal{T}=T_{0}\left[\psi^{-1}(t)\right]$. Choose an arbitrary basis in $\mathcal{T}$, and write it as a matrix in which the basis vectors are written as rows.

(i) By elementary row transformations, reduce this matrix to

$$
\Delta=\left(\begin{array}{cccccccccc}
* & \ldots & * & 0 & & & \ldots & & & 0 \\
* & \ldots & * & * & 0 & \ldots & 0 & & \ldots & 0 \\
* & & \ldots & & * & * & 0 & 0 & \ldots & 0 \\
\vdots & & \vdots & & & & \ddots & \ddots & & 0 \\
* & & & \ldots & & & & * & 0 & 0 \\
* & & & \ldots & & & & * & * & *
\end{array}\right)
$$

where the upper right triangle consists of zeroes, and the last nonzero entries of the rows appear in distinct columns.

(ii) In the matrix $\Delta$ there is the following natural "grading" of the columns corresponding to the grading of the Lie algebra $V$ of vector fields 
on the local tangent cone. Split the columns of $\Delta$ into $M$ blocks $\mathcal{B}_{1}, \ldots, \mathcal{B}_{M}$ such that $\mathcal{B}_{k}$ consists of columns from $\operatorname{dim} H_{k-1}+1$ to $\operatorname{dim} H_{k}$ :

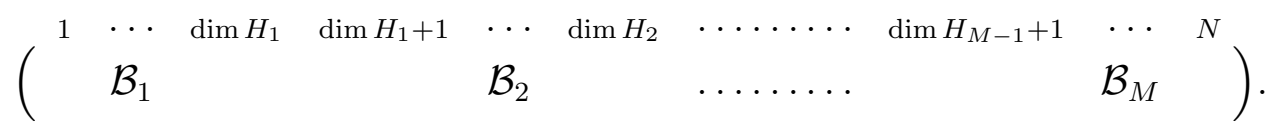

Next, there is also a "grading" of rows of $\Delta$. There are $M$ blocks $\mathcal{A}_{1}, \ldots, \mathcal{A}_{M}$. Here $\mathcal{A}_{l}$ consists of the rows whose last nonzero element appears in a column with index in $\left[\operatorname{dim} H_{l-1}+1, \operatorname{dim} H_{l}\right]$ :

$$
\left(\begin{array}{c}
\mathcal{A}_{1} \\
\mathcal{A}_{2} \\
\vdots \\
\mathcal{A}_{M}
\end{array}\right)=\left(\begin{array}{cccccccccc}
1 & \ldots & \operatorname{dim} H_{1} & \operatorname{dim} H_{1}+1 & \ldots & \operatorname{dim} H_{2} & \ldots & \operatorname{dim} H_{M-1}+1 & \ldots & N \\
& \ldots & * & 0 & & & \ldots & & & 0 \\
* & \ldots & * & * & 0 & \ldots & 0 & & \ldots & 0 \\
* & & \ldots & & * & * & 0 & 0 & \ldots & 0 \\
\vdots & & \vdots & & & & \ddots & \ddots & & 0 \\
* & & & \ldots & & & & * & 0 & 0 \\
* & & & \ldots & & & & * & * & *
\end{array}\right)
$$

Some of these blocks can be empty.

(iii) For each $k=1, \ldots, M$, put $\mathcal{V}_{k}=\mathcal{B}_{k} \cap \mathcal{A}_{k}$

$$
\left(\begin{array}{cccccccccc} 
& \mathcal{V}_{1} & 0 & 0 & & & \ldots & & & 0 \\
* & * & & \mathcal{V}_{2} & 0 & \ldots & 0 & & \ldots & 0 \\
* & & * & & * & & 0 & 0 & \ldots & 0 \\
\vdots & \mathcal{B}_{1} \backslash \mathcal{V}_{1} & \vdots & \mathcal{B}_{2} \backslash \mathcal{V}_{2} & * & & \ddots & \ddots & & 0 \\
* & & & & \vdots & \vdots & * & \mathcal{V}_{M-1} & 0 \\
* & & * & & * & & * & & \mathcal{B}_{M-1} \backslash \mathcal{V}_{M-1} & \mathcal{V}_{M}
\end{array}\right)
$$

Further, we transform the blocks $\mathcal{B}_{k} \backslash \mathcal{V}_{k}, k=1, \ldots, M$. Define the transformation of $\Delta$ by induction. For $k=M$, we have nothing to transform; thus, the base of induction holds.

Suppose that we have transformed the blocks $\mathcal{B}_{k} \backslash \mathcal{V}_{k}, k=l+1, \ldots, M$, $l \leq M-1$, and assume that $\mathcal{V}_{l} \neq \varnothing$ (otherwise, we have nothing to transform). Replace the blocks $\mathcal{A}_{l+1}, \ldots, \mathcal{A}_{M}$ by the projections of their row vectors onto $\left(\operatorname{span}\left\{\mathcal{A}_{l}\right\}\right)^{\perp} \cap \operatorname{span}\left\{\mathcal{A}_{l}, \ldots, \mathcal{A}_{M}\right\}$. Roughly speaking, we remove the part collinear to $\mathcal{A}_{l}$ from the row vectors of $\mathcal{A}_{l+1}, \ldots, \mathcal{A}_{M}$. This projection preserves the blocks $\mathcal{B}_{k} \backslash \mathcal{V}_{k}, k=l+1, \ldots, M, l \leq M-1$, because of the "triangular" structure of $\Delta$.

Moreover, the rows of $\mathcal{B}_{k} \backslash \mathcal{V}_{k}$ are orthogonal to $\mathcal{V}_{k}$ with respect to the classical inner product. 
Denote the resulting vectors by $w_{1}, \ldots, w_{N-\widetilde{N}}$ :

$$
W=\left(\begin{array}{cccccccccc} 
& \mathcal{V}_{1} & 0 & 0 & & & \ldots & & & 0 \\
* & * & & \mathcal{V}_{2} & 0 & \ldots & 0 & & \ldots & 0 \\
* & & * & & * & & 0 & 0 & \ldots & 0 \\
\vdots & \mathcal{V}_{1}^{\perp} & \vdots & \mathcal{V}_{2}^{\perp} & * & & \ddots & \ddots & & 0 \\
* & & & & \vdots & & \vdots & * & \mathcal{V}_{M-1} & 0 \\
* & & * & & * & & * & & \mathcal{V}_{M-1}^{\perp} & \mathcal{V}_{M}
\end{array}\right)=\left(\begin{array}{c}
w_{1} \\
\vdots \\
\vdots \\
w_{N-\widetilde{N}}
\end{array}\right)
$$

Thus, we have constructed a "suitable" basis for $\mathcal{T}$.

Step II. For each $j=1, \ldots, N-\widetilde{N}$, define the number $k(j)$ as follows. Let $l(j)$ be the row index of the last nonzero element of $w_{j}$ in (3.3), and put $k(j)=\operatorname{deg} X_{l(j)}$.

Project each $w_{j}$ onto $\mathcal{V}_{k(j)}$ by taking the last nonzero coordinates belonging to $\mathcal{V}_{k(j)}$, and denote this projection by $p_{j}=\pi_{\mathcal{T}}\left(w_{j}\right)$.

By the properties of $W$, this projection is orthogonal with respect to the standard Riemannian metric on $\mathbb{R}^{N}$ : for each vector $w_{j} \in \mathcal{T}$ we have $\left(w_{j}-\pi_{\mathcal{T}}\left(w_{j}\right)\right) \perp \pi_{\mathcal{T}}(\mathcal{T})$. Indeed, expand

$$
w_{j}=p_{j}+q_{j}=\pi_{\mathcal{T}}\left(w_{j}\right)+q_{j}
$$

and verify that $q_{j}$ is orthogonal to $\pi_{\mathcal{T}}(\mathcal{T})$. Take $u \in \pi_{\mathcal{T}}(\mathcal{T})$. First, suppose that $u$ is a "basis" vector in this image: $u$ is one of the row-vectors of the block $\mathcal{V}_{k}$ for some $1 \leq k \leq M$. If $p_{j} \in \mathcal{V}_{l}$ and $l>k$ then $q_{j} \perp u$ by choice of $W$. If $l \leq k$ then obviously $q_{j} \perp u$ since $q_{j}$ and $u$ have no nonzero coordinates with the same indices. Since $j$ is an arbitrary number and $u$ is an arbitrary basis vector, it follows that all $q_{j}$ are orthogonal to $\pi_{\mathcal{T}}(\mathcal{T})$. Consequently, $\operatorname{span}\left\{q_{j}, j=1, \ldots, N-\tilde{N}\right\} \perp \pi_{\mathcal{T}}(\mathcal{T})$.

Denote by $\pi_{j}$ the vector obtained from $w_{j}$ by putting

$$
\begin{cases}\left(\pi_{j}\right)_{l(j)} & =\left(w_{j}\right)_{l(j)}, \\ \left(\pi_{j}\right)_{k} & =0 \text { for } k \neq l(j) .\end{cases}
$$

Put $\operatorname{deg} \pi_{j}=k(j)$. By construction, the sum of the degrees of $\pi_{1}, \ldots, \pi_{N-\widetilde{N}}$ equals the sum of degrees of the tangent vectors $\left(\theta_{x}^{-1}\right)_{*}\left\langle w_{1}\right\rangle, \ldots,\left(\theta_{x}^{-1}\right)_{*}\left\langle w_{N-\widetilde{N}}\right\rangle$.

Step III. Consider the vectors

$$
\left\{\sigma_{1}, \ldots, \sigma_{\widetilde{N}}\right\}=\left\{e_{1}, \ldots, e_{N}\right\} \backslash\left\{\frac{\pi_{1}}{\left|\pi_{1}\right|}, \ldots, \frac{\pi_{N-\widetilde{N}}}{\left|\pi_{N-\widetilde{N}}\right|}\right\}
$$

of the standard basis in $\mathbb{R}^{N}$. Since $\theta_{x}$ is a diffeomorphism, we have

$$
\operatorname{rank}\left(\left(\left[\left(\theta_{x}\right)_{*}(0)\left\langle\sigma_{i}\right\rangle\right] \varphi\right)(x)\right)_{i=1}^{\widetilde{N}}=\widetilde{N} \Longleftrightarrow \operatorname{rank}\left(\left[\sigma_{i} \psi\right](0)\right)_{i=1}^{\widetilde{N}}=\tilde{N}
$$


Here the symbol $\left(\theta_{x}\right)_{*}(0)\left\langle\sigma_{i}\right\rangle=D \theta_{x}(0)\left\langle\sigma_{i}\right\rangle$ stands for the action of the differential of $\theta_{x}$ at 0 on the vector $\sigma_{i}, i=1, \ldots, \widetilde{N}$.

Verify that the rank of $\left(\left[\sigma_{i} \psi\right](0)\right)_{i=1}^{\widetilde{N}}$ equals $\widetilde{N}$. Indeed, assume the contrary. Then ker $D \psi(0) \cap \operatorname{span}\left\{\sigma_{1}, \ldots, \sigma_{\widetilde{N}}\right\} \neq 0$, and therefore

$$
\operatorname{dim} \mathcal{T} \cap \operatorname{span}\left\{\sigma_{1}, \ldots, \sigma_{\widetilde{N}}\right\} \geq 1 .
$$

Consider the coordinates of $w \in \mathcal{T} \cap \operatorname{span}\left\{\sigma_{1}, \ldots, \sigma_{\widetilde{N}}\right\}$. On the one hand, since $w \in \mathcal{T}$, the choice of the matrix $W$ implies that we can expand $w$ as

$$
w=\sum_{i=1}^{N-\tilde{N}} a_{i} w_{i}
$$

Put $i_{0}=\max \left\{i: a_{i} \neq 0\right\}$. Since $w_{i}=\left(w_{i}-\pi_{i}\right)+\pi_{i}$, it follows that $w=\sum_{i=1}^{N-\widetilde{N}} a_{i}\left(w_{i}-\pi_{i}\right)+a_{i} \pi_{i}=\left(\sum_{i=1}^{i_{0}-1} a_{i}\left[\left(w_{i}-\pi_{i}\right)+\pi_{i}\right]+a_{i_{0}}\left(w_{i_{0}}-\pi_{i_{0}}\right)\right)+a_{i_{0}} \pi_{i_{0}}$.

Recall that by the choice of $\left\{\pi_{i}\right\}_{i=1}^{N-\widetilde{N}}$, the only nonzero coordinate of $\pi_{i_{0}}$ has indexl $\left(i_{0}\right)$, and the nonzero coordinates of

$$
\left(\sum_{i=1}^{i_{0}-1} a_{i}\left[\left(w_{i}-\pi_{i}\right)+\pi_{i}\right]+a_{i_{0}}\left(w_{i_{0}}-\pi_{i_{0}}\right)\right)
$$

have indices strictly less than $l\left(i_{0}\right)$. On the other hand, since $w \in \operatorname{span}\left\{\sigma_{1}, \ldots, \sigma_{\widetilde{N}}\right\}$ it follows from (3.4) that all coefficients of $\pi_{1}, \ldots, \pi_{N-\widetilde{N}}$ in this expansion must vanish. Consequently, $a_{i_{0}}=0$. Thus, we arrive at a contradiction.

Step IV. Verify that the sum of degrees of basis vector fields $\left\{X_{i_{j}}\right\}_{j=1}^{\widetilde{N}}$ such that $X_{i_{j}}(x)=\left(\theta_{x}\right)_{*}(0)\left\langle\sigma_{j}\right\rangle, j=1, \ldots, \widetilde{N}$, equals $\nu_{0}(x)$.

(i) Assume on the contrary that there exist standard vectors $\delta_{1}, \ldots, \delta_{\widetilde{N}}$ with

$$
\nu_{0}(x)=\sum_{i=1}^{\widetilde{N}} \operatorname{deg} \delta_{i}<\sum_{i=1}^{\widetilde{N}} \operatorname{deg} \sigma_{i}
$$

and $\operatorname{rank}\left(\delta_{i} \psi\right)_{i=1}^{\widetilde{N}}=\widetilde{N}$ (here $\operatorname{deg} \delta_{i}$ stands for the value $\operatorname{deg}\left[\left(\theta_{x}\right)_{*}(0)\left\langle\delta_{i}\right\rangle\right]$, $i=1, \ldots, \widetilde{N})$. Since $\left\{\delta_{1}, \ldots, \delta_{\widetilde{N}}\right\} \neq\left\{\sigma_{1}, \ldots, \sigma_{\widetilde{N}}\right\}$, there exists at least one $\frac{\pi_{j}}{\left|\pi_{j}\right|}=\delta_{k}$ for some $j$ and $k$. Consequently, there exists a vector $v$ satisfying $v+\frac{\pi_{j}}{\left|\pi_{j}\right|} \in \mathcal{T}$. Moreover, the index of the last nonzero component of $v$ is strictly less than $l(j)$ (see the matrix $W$ of (3.3)). 
(ii) Consider the chosen vector $v$. It is evident that $v \notin \mathcal{T}$ (otherwise, $\pi_{j} \in \mathcal{T}$ and $\delta_{k} \in \mathcal{T}$, which is impossible). Observe that $\operatorname{rank}\left(\tau_{i} \psi\right)_{i=1}^{\widetilde{N}}<\widetilde{N}$, where $\tau_{k}=\frac{1}{2}\left(v+\frac{\pi_{j}}{\left|\pi_{j}\right|}\right) \in \mathcal{T}$, and $\tau_{i}=\delta_{i}$ for all $i \neq k$. Consequently, $\operatorname{rank}\left(\lambda_{i} \psi\right)_{i=1}^{\widetilde{N}}=\tilde{N}$, where $\lambda_{k}=v$ and $\lambda_{i}=\delta_{i}$ for all $i \neq k$. Indeed, assume on the contrary that $\operatorname{rank}\left(\lambda_{i} \psi\right)_{i=1}^{\widetilde{N}}<\tilde{N}$. Then

$$
\left(\delta_{i} \psi\right)_{i=1}^{\widetilde{N}}=2\left(\tau_{i} \psi\right)_{i=1}^{\widetilde{N}}-\left(\lambda_{i} \psi\right)_{i=1}^{\widetilde{N}} .
$$

The assumption implies that $v \psi=\sum_{i=1}^{\widetilde{N}} a_{i}\left[\delta_{i} \psi\right]$ because $\operatorname{rank}\left(\delta_{i} \psi\right)_{i=1}^{\widetilde{N}}=\widetilde{N}$. Since $2 \tau_{k} \psi=\left(v+\frac{\pi_{j}}{\left|\pi_{j}\right|}\right) \psi=0$, it follows from (3.6) that the column $k$ of $\left(\delta_{i} \psi\right)_{i=1}^{\widetilde{N}}$ is equal to $-v \psi=-\sum_{i=1}^{\widetilde{N}} a_{i}\left[\delta_{i} \psi\right]$, and, consequently, $\operatorname{rank}\left(\delta_{i} \psi\right)_{i=1}^{\widetilde{N}}<$ $\widetilde{N}$. Thus, we arrive at a contradiction.

Since $\operatorname{rank}\left(\lambda_{i} \psi\right)_{i=1}^{\widetilde{N}}=\widetilde{N}$, assuming the contrary and applying similar arguments, we infer that there exists at least one coordinate $(v)_{j}$ of $v$ such that $\operatorname{rank}\left(\mu_{i} \psi\right)_{i=1}^{\widetilde{N}}=\tilde{N}$, where $\mu_{k}=e_{j}$ and $\mu_{i}=\delta_{i}$ for all $i \neq k$.

(iii) Put

$$
l=\min \left\{j: \operatorname{rank}\left(\mu_{i} \psi\right)_{i=1}^{\widetilde{N}}=\tilde{N}, \text { where } \mu_{k}=e_{j}, \mu_{i}=\delta_{i} \text { for } i \neq k\right\} .
$$

Note that $l$ is strictly less than $l(j)$ (the index of the only nonzero coordinate of $\pi_{j}$ ). If $\operatorname{deg} e_{l}<\operatorname{deg} \pi_{j}$ then we obtain a contradiction with the assumption that $\sum_{i=1}^{\widetilde{N}} \operatorname{deg} \delta_{i}=\nu_{0}(x)$. If $\operatorname{deg} e_{l}=\operatorname{deg} \pi_{j}$ then we consider the collection $\left\{\mu_{i}\right\}_{i=1}^{\widetilde{N}}$ instead of $\left\{\delta_{i}\right\}_{i=1}^{\widetilde{N}}$, and repeat the previous arguments.

(iv) Observe that after finitely many iterations of the arguments described in substeps (ii)-(iii), we obtain a collection satisfying

$$
\sum_{i=1}^{\widetilde{N}} \operatorname{deg} \mu_{i}<\sum_{i=1}^{\widetilde{N}} \operatorname{deg} \delta_{i}=\nu_{0}(x)
$$

(here $\operatorname{deg} \mu_{i}$ stands for the value $\left.\operatorname{deg}\left[\left(\theta_{x}\right)_{*}(0)\left\langle\mu_{i}\right\rangle\right], i=1, \ldots, \widetilde{N}\right)$. This contradicts to (3.5).

Step V. (i) Denote by $\mathcal{S}$ the plane $\operatorname{span}\left\{p_{1}, \ldots, p_{N-\widetilde{N}}\right\}$, where the vectors $p_{1}, \ldots, p_{N-\widetilde{N}}$ are defined in Step II, and consider $\mathcal{S} \cap \operatorname{Box}_{2}(0, r)$. Without loss of generality we may assume that $p_{1}, \ldots, p_{N-\widetilde{N}}$ are orthonormal (it suffices to carry out the procedure described in Step II within each $\left.\mathcal{V}_{i}, i=1, \ldots, M\right)$. Verify that this intersection equals a direct product of balls of radii $r^{k}, k=$ $1, \ldots, M$, in some basis. 
To this end, we consider a new orthonormal basis $\mathcal{O} e_{1}, \ldots, \mathcal{O} e_{N}$ in $\mathbb{R}^{N}$, where the orthogonal transformation $\mathcal{O}$ satisfies $\mathcal{O}\left(\operatorname{span}\left\{\mathcal{V}_{i}\right\}\right)=\operatorname{span}\left\{\mathcal{V}_{i}\right\}$, $i=1, \ldots, M$, and the image of each $p_{j}$ is the standard basis vector $e_{i_{j}}$, $j=1, \ldots, N-\widetilde{N}$. Then the $d_{2}$-ball in the initial basis coincides with the one in the new metric: $\mathcal{O}\left(\operatorname{Box}_{2}(0, r)\right)=\operatorname{Box}_{2}(0, r)$. Thus, we have constructed a basis for $\mathcal{S}$ consisting of $N-\widetilde{N}$ standard basis vectors.

Next, $\mathcal{O}\left(\operatorname{Box}_{2}(0, r) \cap \mathcal{S}\right)=\operatorname{Box}_{2}(0, r) \cap \mathcal{O}(\mathcal{S})=\operatorname{Box}_{2}^{\mathcal{O}(\mathcal{S})}(0, r)$, where the latter is a ball in the metric $\left.d_{2}\right|_{\mathcal{O}(\mathcal{S})}$. By the choice of $\mathcal{O}$, it equals a direct product of Euclidean balls.

(ii) Obviously, the $(N-\widetilde{N})$-dimensional Lebesgue measure of $\operatorname{Box}_{2}^{\mathcal{O}(\mathcal{S})}(0, r)$ equals $C r^{\nu-\nu_{0}(x)}$, where $C$ depends only on $x$. Since $\mathcal{O}$ is an orthogonal transformation, so is the inverse mapping $\mathcal{O}^{-1}$, and the $(N-\widetilde{N})$-dimensional Lebesgue measure of $\operatorname{Box}_{2}(0, r) \cap \mathcal{S}=\mathcal{O}^{-1}\left(\operatorname{Box}_{2}^{\mathcal{O}(\mathcal{S})}(0, r)\right)$ is the same; thus, it also equals $C r^{\nu-\nu_{0}(x)}$.

Observe that at regular points the $(N-\widetilde{N})$-dimensional Lebesgue measure of $\mathcal{S} \cap \operatorname{Box}_{2}(0, r)$ equals $\prod_{k=1}^{M} \omega_{n_{k}-\tilde{n}_{k}} r^{\nu-\tilde{\nu}}$.

Step VI. On this step we show that there exists $r_{0}>0$ such that for $r \leq r_{0}$ the set

$$
\pi_{\mathcal{T}}\left(\mathcal{T} \cap \operatorname{Box}_{2}(0, r)\right)
$$

coincides with the $o(r)$-neighborhood of $\mathcal{S} \cap \operatorname{Box}_{2}(0, r)$ in $\mathcal{S}$, where $o(r)$ is taken with respect to the metric $d_{2}$. From this, we deduce that the $\mathcal{H}^{N-\widetilde{N}_{-}}$ measure distortion of $\pi_{\mathcal{T}}$ equals

$$
\lim _{r \rightarrow 0} \frac{\mathcal{H}^{N-\widetilde{N}}\left(\pi_{\mathcal{T}}\left(\mathcal{T} \cap \operatorname{Box}_{2}(0, r)\right)\right)}{\mathcal{H}^{N-\widetilde{N}}\left(\mathcal{T} \cap \operatorname{Box}_{2}(0, r)\right)}=\lim _{r \rightarrow 0} \frac{\mathcal{H}^{N-\widetilde{N}}\left(\mathcal{S} \cap \operatorname{Box}_{2}(0, r)\right)}{\mathcal{H}^{N-\widetilde{N}}\left(\mathcal{T} \cap \operatorname{Box}_{2}(0, r)\right)} .
$$

(i) Fix a vector $w_{j}$. Show that there exists $r_{0 j}>0$ such that the length of $\mathbb{R} w_{j} \cap \operatorname{Box}_{2}(0, r)$ equals $O\left(r^{k(j)}\right)$ for all $r \in\left(0, r_{0 j}\right)$. Indeed, fix $r_{0 j}>0$ and a take point $\bar{u} \in \mathbb{R}_{+} w_{j} \cap \partial \operatorname{Box}_{2}\left(x, r_{0 j}\right)$ and the corresponding vector $u$ whose coordinates coincide with those of $\bar{u}$. Here

$$
\mathbb{R}_{+} w_{j}=\left\{z \in \mathbb{R}^{N}: z=a w_{j}, a \in \mathbb{R}_{+}\right\}
$$

Then $u=\sum_{k=1}^{k(j)} u_{k}$, where

$$
u_{k}=u_{k}\left(r_{0 j}\right) \in \operatorname{span}\left\{\mathcal{V}_{k}\right\}
$$

and $d_{2}(0, \bar{u})=r_{0 j}$. Consequently, $\left|u_{k}\left(r_{0 j}\right)\right| \leq\left(r_{0 j}\right)^{k}$; here $|\cdot|$ denotes the Euclidean length. 
We verify that $\left|u_{k}(r)\right|=O\left(r^{k(j)}\right)$ for all $r \in\left(0, r_{0 j}\right), k=1, \ldots, k(j)$. The vector $u(r)=\sum_{k=1}^{k(j)} u_{k}(r)$ has the same coordinates as the point $\mathbb{R}_{+} w_{j} \cap$ $\partial \operatorname{Box}_{2}(0, r)$. Assume on the contrary that for every $r_{0 j}$ and for every $K<\infty$ there exist $r<r_{0 j}$ and $u_{l}(r)$ with

$$
\left|u_{l}(r)\right|>K r^{k(j)} .
$$

Fix such $r_{0}, K$ and $r<r_{0}$, and put $\alpha_{r}=\frac{|u|}{|u(r)|}$. Then we can represent $u(r)$ as

$$
u(r)=\alpha_{r} u=\sum_{k=1}^{k(j)} \alpha_{r} u_{k}=\sum_{k=1}^{k(j)} u_{k}(r) .
$$

On the one hand, $K r^{k(j)}<\left|u_{l}(r)\right|=\left|\alpha_{r} u_{l}\right|=\left|\alpha_{r}\right|\left|u_{l}\right|$. Therefore,

$$
\left|\alpha_{r}\right|>K \frac{r^{k(j)}}{\left|u_{l}\right|}
$$

On the other hand, $k=k(j)$, in view of its definition, satisfies $\left|u_{k(j)}(r)\right| \leq$ $L r^{k(j)}$ for all $r \in\left(0, r_{0}\right]$. Since $u_{k(j)}(r)=\alpha_{r} \cdot u_{k(j)}$, we have

$$
L r^{k(j)} \geq\left|u_{k(j)}(r)\right|=\left|\alpha_{r}\right| \cdot\left|u_{k(j)}\right|>K r^{k(j)} \frac{\left|u_{k(j)}\right|}{\left|u_{l}\right|} .
$$

Observe that $\frac{\left|u_{k(j)}\right|}{\left|u_{l}\right|}$ depends only on $w_{j}$, and is independent of $r>0$. Obviously, the equality

$$
L r^{k(j)}>K r^{k(j)}
$$

is violated for $K>L$. Thus we arrive at a contradiction. Consequently, $\left|u_{k}\right|=O\left(r^{k(j)}\right), r \in\left(0, r_{0}\right)$, for all $k=1, \ldots, k(j)$.

(ii) Consider $\mathcal{T} \cap \operatorname{Box}_{2}(0, r)$, and the projection $\pi_{\mathcal{T}}: \mathcal{T} \rightarrow \mathcal{S}$. By the properties of the matrix $W$ (see (3.3) $)$, it is bijective. Verify that

$$
d_{2}\left(0, \partial\left[\pi_{\mathcal{T}}\left(\mathcal{T} \cap \operatorname{Box}_{2}(0, r)\right)\right]\right)=r(1+o(1)),
$$

where $\partial$ stands for the boundary relative to the plane $\mathcal{T}$. First, consider the basis elements $w$ in $\mathcal{T}$. Represent each of them as $w=y+z$, where $\pi_{\mathcal{T}}(y+z)=y$. The properties of $\pi_{\mathcal{T}}$ yield $\operatorname{deg} z<\operatorname{deg} y$, where the degree of the vector $u=\sum_{i=1}^{\widetilde{N}} u_{i} e_{i}$ is defined as

$$
\operatorname{deg} u=\operatorname{deg}\left(\sum_{i=1}^{\widetilde{N}} u_{i} X_{i}\right)=\max _{i=1, \ldots, N}\left\{\operatorname{deg} X_{i}: u_{i} \neq 0\right\} .
$$


If $w \in \mathcal{T}$ is not a basis vector, then we expand it in the basis $\left\{w_{i}\right\}_{i=1}^{N-\widetilde{N}}$ as

$$
w=\sum_{k=1}^{N-\widetilde{N}} a_{k} w_{k}=\sum_{k=1}^{N-\widetilde{N}} a_{k} y_{k}+\sum_{k=1}^{N-\widetilde{N}} a_{k} z_{k}
$$

where $\pi_{\mathcal{T}}\left(w_{k}\right)=y_{k}, k=1, \ldots, N-\widetilde{N}$. Next, it suffices to note that

$$
\operatorname{deg}\left(\sum_{k=1}^{N-\widetilde{N}} a_{k} y_{k}\right)>\operatorname{deg}\left(\sum_{k=1}^{N-\widetilde{N}} a_{k} z_{k}\right) .
$$

Thus, we can represent every $w \in \mathcal{T}$ as $w=y+z$, where $\pi_{\mathcal{T}}(w)=y$ and $\operatorname{deg} y>\operatorname{deg} z$.

Define a new quasimetric $d_{2 E}^{0}$ on $\operatorname{Box}_{2}(0, r)$. For $v, w \in \operatorname{Box}_{2}(0, r)$ put $d_{2 E}^{0}(v, w)=d_{2}(0, w-v)$, where $w-v$ denotes the Euclidean difference. This definition implies that $\operatorname{Box}_{2}(0, r)$ coincides with the ball $\operatorname{Box}_{2 E}(0, r)$ of radius $r$ centered at 0 in the metric $d_{2 E}^{0}$. Consequently, for proving (3.7), it suffices to show that

$$
d_{2 E}^{0}\left(0, \partial\left[\pi_{\mathcal{T}}\left(\mathcal{T} \cap \operatorname{Box}_{2}(0, r)\right)\right]\right)=r(1+o(1)),
$$

where $\partial$ stands for the boundary relative to the plane $\pi_{\mathcal{T}}(\mathcal{T})$. Take $w=y+z$ with $d_{2}(0, w)=r$ and $\operatorname{deg} y>\operatorname{deg} z$. Then

$$
d_{2}(0, y+z)=d_{2 E}^{0}(0, y+z)=r,
$$

$\rho(0, y+z)=O\left(r^{\operatorname{deg} y}\right)\left(\right.$ see Step $\mathbf{V}$, substep (i)), and $\rho(0, y)=O\left(r^{\operatorname{deg} y}\right)$ since $\pi_{\mathcal{T}}$ is a linear mapping. Then, we have $\rho(y, y+z)=O\left(r^{\operatorname{deg} y}\right)$ because $y \perp z$.

Observe that $d_{2 E}^{0}(y, y+z)=d_{2}(0, z)$. Represent $z$ as $z=\sum_{i=1}^{I(z)} z_{i}$, where $z_{i} \in \mathcal{V}_{i}$. Here $\operatorname{deg} z_{I(z)}=I(z)<\operatorname{deg} y$ because $\operatorname{deg} z<\operatorname{deg} y$. Since

$$
O\left(r^{\operatorname{deg} y}\right)=\rho(y, y+z) \sim \max _{\substack{i=1, \ldots, I(z) \\ j=1, \ldots, \operatorname{dim} H_{i} / H_{i-1}}}\left\{\left|\left(z_{i}\right)_{j}\right|\right\}
$$

it follows that $\left|z_{i}\right| \leq O\left(r^{\operatorname{deg} y}\right)$.

This implies that

$$
\begin{aligned}
d_{2}(0, z)=\max _{i=1, \ldots, I(z)}\left\{\left|z_{i}\right|^{\frac{1}{i}}\right\} \leq \max _{i=1, \ldots, I(z)} & \left\{\left|z_{i}\right|^{\frac{1}{I(z)}}\right\} \\
& \leq O\left(r^{\frac{\operatorname{deg} y}{I(z)}}\right) \leq O\left(r^{1+\frac{1}{M-1}}\right)=r \cdot o(1),
\end{aligned}
$$


where $o(1)$ is at most $\mathcal{C} r^{\frac{1}{M-1}}$ for some $0<\mathcal{C}<\infty$ independent of the point of $\operatorname{Box}_{2}(0, r)$.

Proposition 3.3 implies that $d_{2 E}^{0}(v, w) \leq d_{2 E}^{0}(v, u)+c d_{2 E}^{0}(u, w)$ for $u, v, w \in$ $\operatorname{Box}_{2}(0, r)$ and some $c>0$ (it suffices to put $\widehat{F}_{\mu, \beta}^{i}(x) \equiv 0$ in Proposition 3.3). Consequently,

$$
d_{2}(0, y)=d_{2 E}^{0}(0, y) \leq d_{2 E}^{0}(0, z+y)+c d_{2 E}^{0}(y, z+y)=r(1+o(1)),
$$

and (3.7) follows.

Thus, the measure of $\mathcal{T} \cap \operatorname{Box}_{2}(x, r)$ is equivalent to $C r^{\nu-\nu_{0}(x)}$ as $r \rightarrow 0$. The theorem follows.

Definition 3.9. Let $\xi: \mathbb{M} \rightarrow \widetilde{\mathbb{M}}$ be a mapping of two Carnot manifolds. Fix $x \in \mathbb{M}$. The $d_{2}$-distortion of $\xi$ at $y$ with respect to $x$ equals

$$
\frac{d_{2}(\xi(y), \xi(x))}{d_{2}(y, x)}
$$

and the $\rho$-distortion equals

$$
\frac{\rho(\xi(y), \xi(x))}{\rho(y, x)}
$$

To simplify notation, we denote the Gram determinant $\sqrt{\operatorname{det}\left(A A^{*}\right)}$ for a matrix $A$ by $\mathcal{D}(A)$. We denote the Gram determinant $\sqrt{\operatorname{det}\left(B^{*} B\right)}$ for a matrix $B$ by $\widetilde{\mathcal{D}}(B)$.

Proposition 3.10. The matrix of the differential of the mapping $\theta_{x}, x \in \mathbb{M}$, at 0 equals the identity matrix.

Henceforth we denote the Riemann tensor at $y$ by $g(y)$.

Theorem 3.11. Suppose that $x \in \varphi^{-1}(t)$ is a regular point. Then

(I) In a neighborhood of $0=\theta_{x}^{-1}(x)$ there exists a mapping from $T_{0}\left[\psi^{-1}(t)\right] \cap$ $\operatorname{Box}_{2}(0, r(1+o(1)))$ to $\psi^{-1}(t) \cap \operatorname{Box}_{2}(0, r)$ such that both $d_{2}$-and $\rho$-distortions with respect to 0 are equal to $1+o(1)$ at every $y \in T_{0}\left[\psi^{-1}(t)\right] \cap \operatorname{Box}_{2}(0, r(1+$ $o(1))$ ), where $o(1)$ is uniform in $x=\theta_{x}(0) \in \mathcal{U} \Subset \mathbb{M}$ and in $y \in T_{0}\left[\psi^{-1}(t)\right] \cap$ $\operatorname{Box}_{2}(0, r(1+o(1)))$.

(II) The $\mathcal{H}^{N-\widetilde{N}_{-}}$-measure of $\varphi^{-1}(t) \cap \operatorname{Box}_{2}(x, r)$ equals

$$
\widetilde{\mathcal{D}}\left(\left.g\right|_{\operatorname{ker} D \varphi(x)}\right) \cdot \prod_{k=1}^{M} \omega_{n_{k}-\tilde{n}_{k}} \cdot \frac{\mathcal{D}(D \varphi(x))}{\mathcal{D}(\widehat{D} \varphi(x))} r^{\nu-\tilde{\nu}}(1+o(1))
$$

with $o(1) \rightarrow 0$ as $r \rightarrow 0$, where $o(1) \rightarrow 0$ uniformly in $x \in \mathcal{U} \Subset \mathbb{M}$. 
In the proof of Theorem 3.11 we use the following notation introduced in Theorem 3.7. We denote the mapping $\varphi \circ \theta_{x}$ by $\psi$, and the tangent space $T_{0}\left[\psi^{-1}(t)\right]$ by $\mathcal{T}$. We also use the mapping $\pi_{\mathcal{T}}$ defined in Step III and the image $\mathcal{S}=\pi_{\mathcal{T}}(\mathcal{T})$.

Proof of Theorem 3.11. Notice that without loss of generality we may assume that $\widehat{D} \psi(z)$ is strictly separated from 0 on $(\text { ker } \widehat{D} \psi(0))^{\perp}$ for $z \in \operatorname{Box}_{2}(0, r)$, i. e., $\widehat{D} \psi(z)(v) \geq \alpha>0$ for all $z \in \operatorname{Box}_{2}(0, r)$ and $v \in(\operatorname{ker} \widehat{D} \psi(0))^{\perp}$ with $|v|=1$ (here $|\cdot|$ denotes the Euclidean norm).

Step I. Verify that $C$ in $(3.2)$ at a regular point equals $\frac{\mathcal{D}(D \varphi(x))}{\mathcal{D}(\widehat{D} \varphi(x))}$ up to a Riemannian factor. In particular, we show that $\frac{\mathcal{D}(D \varphi(x))}{\mathcal{D}(\widehat{D} \varphi(x))}$ equals the measure distortion under the mapping $\pi_{\mathcal{T}}$ defined in Step II of Theorem 3.7.

To this end, consider the projection of the normal space $\mathcal{N}$ to $\mathcal{T}$ onto $H_{\widetilde{M}}(0)$ constructed by the choice of vectors written as rows in the matrix of $\widehat{D} \psi(0)$ (recall that the matrix of $D \psi(0)$ equals the matrix of $\left(\left[\left(\theta_{x}^{-1}\right)_{*} X_{1}\right] \psi, \ldots,\left[\left(\theta_{x}^{-1}\right)_{*} X_{N}\right] \psi\right)$, and in the bases $\left\{e_{i}\right\}_{i=1}^{N}$ and $\left\{e_{i}\right\}_{i=1}^{\widetilde{N}}$ the matrices of $D \psi(0)$ and $\widehat{D} \psi(0)$ have the structure similar to (2.4) and (2.5), respectively). Denote this projection by $\pi_{\mathcal{N}}$ and its image by $\mathcal{L}=\pi_{\mathcal{N}}(\mathcal{N})$. Verify that $\mathcal{S}$ is orthogonal to $\mathcal{L}$. Write a basis for $\mathcal{N}$ as the row vectors of the matrix $D \psi(0)$ in the basis $\left\{e_{i}\right\}_{i=1}^{N}$.

(i) Without loss of generality we may assume that $\pi_{\mathcal{N}}$ is an orthogonal projection (see Theorem 3.7). Indeed, the matrix of $D \psi(0)$ admits "gradings" $\widetilde{\mathcal{B}}_{1}, \ldots, \widetilde{\mathcal{B}}_{\widetilde{M}}$ of the columns and $\widetilde{\mathcal{A}}_{1}, \ldots, \widetilde{\mathcal{A}}_{\widetilde{M}}$ of the rows similar to those of the matrix $\Delta$ of Step II substep (ii) of Theorem 3.7. Namely, we say that a column vector belongs to the block $\widetilde{\mathcal{B}}_{k}$ if its index is at least $\operatorname{dim} H_{k-1}+1$ (here we assume that $\operatorname{dim} H_{0}=0$ ) and at most $\operatorname{dim} H_{k}, k=1, \ldots, \widetilde{M}$, and we say that a row vector belongs to the block $\widetilde{\mathcal{A}}_{l}$ if the index of its first nonzero element is at least $\operatorname{dim} H_{k-1}+1$ (here we assume that $\operatorname{dim} H_{0}=0$ ) and at most $\operatorname{dim} H_{k}, k=1, \ldots, \widetilde{M}$. Put $\widetilde{\mathcal{V}}_{k}=\widetilde{\mathcal{B}}_{k} \cap \widetilde{\mathcal{A}}_{k,}, k=1, \ldots, \widetilde{M}$.

Next, we transform the blocks $\widetilde{\mathcal{B}}_{k} \backslash \widetilde{\mathcal{V}}_{k}, k=1, \ldots, \widetilde{M}$. Define the required transformation of the matrix of $D \psi(0)$ by induction. For $k=1$, we have nothing to transform; thus, the base of induction holds. Suppose that we have transformed the blocks $\widetilde{\mathcal{B}}_{k} \backslash \widetilde{\mathcal{V}}_{k}, k=1, \ldots, l, l \geq 1$. Assume that $\widetilde{\mathcal{V}}_{l+1} \neq \varnothing$ (otherwise, the transformation is trivial). Replace the blocks $\widetilde{\mathcal{A}}_{1}, \ldots, \widetilde{\mathcal{A}}_{l}$ by the projections of their row vectors onto the space

$$
\left(\operatorname{span}\left\{\mathcal{A}_{l+1}\right\}\right)^{\perp} \cap \operatorname{span}\left\{\widetilde{\mathcal{A}}_{1}, \ldots, \widetilde{\mathcal{A}}_{l+1}\right\} .
$$

This projection leaves the blocks $\widetilde{\mathcal{B}}_{k} \backslash \widetilde{\mathcal{V}}_{k}, k=1, \ldots, l, l \geq 1$, unchanged because of the "triangular" structure of the matrix of $D \psi(0)$. 
Moreover, the rows of the block $\widetilde{\mathcal{B}}_{k} \backslash \mathcal{V}_{k}$ are orthogonal to those of $\widetilde{\mathcal{V}}_{k}$, $k=1, \ldots, \widetilde{M}$. It results

$$
\left(\begin{array}{ccccccccccccc}
\mathcal{V}_{1} & \mathcal{V}_{2}^{\perp} & & \mathcal{V}_{3}^{\perp} & & * & * & & \vdots & \vdots & * & \ldots & * \\
0 & \mathcal{V}_{2} & & & \vdots & \vdots & * & & * & * & \ldots & * \\
0 & 0 & & \mathcal{V}_{3} & & & & * & \mathcal{V}_{\overparen{M}-1}^{\perp} & \mathcal{V}_{M}^{\perp} & * & \ldots & * \\
\vdots & \vdots & 0 & 0 & & \ddots & \ddots & \ddots & * & & * & \ldots & * \\
0 & 0 & & & \vdots & 0 & 0 & 0 & \mathcal{V}_{\widetilde{M}-1} & & * & \ldots & * \\
0 & 0 & 0 & 0 & 0 & 0 & 0 & 0 & 0 & \mathcal{V}_{\widetilde{M}} & * & \ldots & *
\end{array}\right)
$$

By the construction, the blocks $\mathcal{V}_{1}, \ldots, \mathcal{V}_{\widetilde{M}}$ constitute the matrix of $\widehat{D} \psi(0)$ (see (2.5)), and $\operatorname{dim} \mathcal{L}=\widetilde{N}$ at regular points.

(ii) It is easy to see that $\mathcal{L}$ is orthogonal to $\mathcal{S}$.

Indeed, take two vectors $v \in \mathcal{L}$ and $w \in \mathcal{S}$. First, suppose that $v$ and $w$ are the images of arbitrary basis vectors in $\mathcal{N}$ and $\mathcal{T}$ respectively. Then $v \in \widetilde{\mathcal{V}}_{i}$ and $w \in \mathcal{V}_{j}$ for some $i$ and $j$. If $i \neq j$ then obviously $v \perp w$ (since they have no corresponding nonzero components).

Suppose that $i=j$ and consider the preimages of $v$ and $w$. By construction, they are orthogonal. Note that the preimage of $v$ belongs to $\mathcal{N}$, and we can write it as $(0, \ldots, 0, \tilde{v}, *)$, where the nonzero part begins with some element $\tilde{v}$ of $v$. The preimage of $w$ belongs to $\mathcal{T}$, and it can be written as $(*, \tilde{w}, 0, \ldots, 0)$, where the last component of the nonzero part is some element $\tilde{w}$ of $w$. Consequently,

$$
0=(0, \ldots, 0, \tilde{v}, *) \cdot(*, \tilde{w}, 0, \ldots, 0)^{T}=(v, w)
$$

and $v \perp w$.

Since $v$ and $w$ are the images of arbitrary basis vectors in $\mathcal{T}$ and $\mathcal{N}$, we have $\mathcal{S} \perp \mathcal{L}$.

(iii) Thus, $\mathcal{S}$ is orthogonal to $\mathcal{L}$. Moreover, the projection of $\mathcal{T}$ onto $\mathcal{S}$ is also orthogonal (see Theorem [3.7): $\left(v-\pi_{\mathcal{T}}(v)\right) \perp \pi_{\mathcal{T}}(v)$ for all $v \in \mathcal{T}$. Let us verify that the measure distortions under the projections $\pi_{\mathcal{T}}$ and $\pi_{\mathcal{N}}$ coincide.

Step II. (i) Show that

$$
\operatorname{dim} \mathcal{T}-\operatorname{dim}(\mathcal{T} \cap \mathcal{S})=\operatorname{dim} \mathcal{N}-\operatorname{dim}(\mathcal{N} \cap \mathcal{L})
$$

Put $l=\operatorname{dim}(\mathcal{T} \cap \mathcal{S})$. On the one hand, since $\mathcal{N} \perp \mathcal{T}$ and $\mathcal{L} \perp \mathcal{S}$, it follows that $\operatorname{span}\{\mathcal{N}, \mathcal{L}\} \perp(\mathcal{T} \cap \mathcal{S})$, and so $\operatorname{span}\{\mathcal{N}, \mathcal{L}\} \subset(\mathcal{T} \cap \mathcal{S})^{\perp}$. On the other hand, if $v \perp(\mathcal{T} \cap \mathcal{S})$ then $v \in \operatorname{span}\{\mathcal{N}, \mathcal{L}\}$. Indeed, assume on the contrary 
that $v \notin \operatorname{span}\{\mathcal{N}, \mathcal{L}\}$. Then $v=v_{\mathcal{N L}}+v_{(\mathcal{N L})^{\perp}}$, where $v_{\mathcal{N} \mathcal{L}} \in \operatorname{span}\{\mathcal{N}, \mathcal{L}\}$ and $v_{(\mathcal{N L})^{\perp}} \in(\operatorname{span}\{\mathcal{N}, \mathcal{L}\})^{\perp}$ with $v_{(\mathcal{N} \mathcal{L})^{\perp}} \neq 0$. Consequently, since $v_{(\mathcal{N} \mathcal{L})^{\perp}} \perp \mathcal{N}$ and $v_{(\mathcal{N L})^{\perp}} \perp \mathcal{L}$, we obtain $v_{(\mathcal{N} \mathcal{L})^{\perp}} \in(\mathcal{T} \cap \mathcal{S})$ and arrive at a contradiction.

Thus, $\operatorname{span}\{\mathcal{N}, \mathcal{L}\}=(\mathcal{T} \cap \mathcal{S})^{\perp}$, and $\operatorname{dim} \operatorname{span}\{\mathcal{N}, \mathcal{L}\}=N-l$. Since $\operatorname{dim} \mathcal{N}=\operatorname{dim} \mathcal{L}$, we get

$$
\operatorname{dim}(\mathcal{N} \cap \mathcal{L})=2 \operatorname{dim} \mathcal{N}-\operatorname{dim} \operatorname{span}\{\mathcal{N}, \mathcal{L}\}=2 \widetilde{N}-N+l
$$

Consequently,

$$
\operatorname{dim} \mathcal{T}-\operatorname{dim}(\mathcal{T} \cap \mathcal{S})=N-\tilde{N}-l=\operatorname{dim} \mathcal{N}-\operatorname{dim}(\mathcal{N} \cap \mathcal{L})
$$

(ii) Recall that $\mathcal{L}=\operatorname{ker} \widehat{D} \psi(0)$ at regular points (see Step I, substep (ii)). Verify that $\operatorname{dim}(\mathcal{T} \cap \mathcal{L})=\operatorname{dim}(\mathcal{N} \cap \mathcal{S})=0$. Indeed, assume on the contrary that there exists $h \in \mathcal{T} \cap \mathcal{L}, h \neq 0$. Then, $h \perp \mathcal{N}$ and $h \perp \mathcal{S}$. Since $(\mathcal{T} \cap \mathcal{L})^{\perp}=$ $\operatorname{span}\{\mathcal{N}, \mathcal{S}\}$, which we can justify similarly to the substep (i) above, it follows that $\operatorname{dim} \operatorname{span}\{\mathcal{N}, \mathcal{S}\} \leq N-1$. Consequently, from $\operatorname{dim} \operatorname{span}\{\mathcal{N}\}=\widetilde{N}$ and $\operatorname{dim} \operatorname{span}\{\mathcal{S}\}=N-\widetilde{N}$, we infer that $\operatorname{dim}(\mathcal{N} \cap \mathcal{S}) \geq 1$ and there exists $h^{\perp} \in(\mathcal{N} \cap \mathcal{S})$; thus, $\widehat{D} \psi(0)$ vanishes on some vector in $\mathcal{N}$. This implies that one of $\widetilde{N}$ vectors in $\mathcal{N}$ is orthogonal to $\mathcal{L}$. Consequently, its image under $\pi_{\mathcal{N}}$ vanishes since every vector in $\mathcal{N}$ can be uniquely represented as a sum of a vector in $\mathcal{L}$ and a vector in $\mathcal{L}^{\perp}$. Thus, $\pi_{\mathcal{N}}$ has a nonzero kernel, and $\operatorname{dim}\left(\pi_{\mathcal{N}}(\mathcal{N})\right)<\widetilde{N}$. This implies that $\operatorname{dim}\left[\pi_{\mathcal{N}}(\mathcal{N})\right]=\operatorname{dim}\left[(\operatorname{ker} \widehat{D} \psi(0))^{\perp}\right]<\widetilde{N}$ which contradicts the regularity of $x$.

Hence, $\operatorname{dim}(\mathcal{T} \cap \mathcal{L})=0$ and $\operatorname{dim}(\mathcal{N} \cap \mathcal{S})=0$.

(iii) Put $q=\operatorname{dim} \mathcal{T}-\operatorname{dim}(\mathcal{T} \cap \mathcal{S})$. Consider the maximal subset $\mathcal{S}^{\prime}$ of $\mathcal{S}$ satisfying $\mathcal{S}^{\prime} \perp(\mathcal{T} \cap \mathcal{S})$. Namely, $\mathcal{S}^{\prime}=(\mathcal{T} \cap \mathcal{S})^{\perp} \cap \mathcal{S}$. Then $\operatorname{dim} \mathcal{S}^{\prime}=q$. Put $\mathcal{T}^{\prime}=\pi_{\mathcal{T}}^{-1}\left(\mathcal{S}^{\prime}\right)$. It is easy to see that $\mathcal{T}^{\prime} \perp(\mathcal{T} \cap \mathcal{S})$. Indeed, take arbitrary vectors $v \in \mathcal{T} \cap \mathcal{S}, v^{\prime} \in \mathcal{S}^{\prime}$, and a vector $w$, such that $v^{\prime}+w=\pi_{\mathcal{T}}^{-1}\left(v^{\prime}\right)$. In particular, $v^{\prime}+w \in \mathcal{T}$. Note that $w \perp(\mathcal{T} \cap \mathcal{S})$ because $\pi_{\mathcal{T}}$ is an orthogonal projection. Then $\mathcal{T} \ni \pi_{\mathcal{T}}^{-1}\left(v^{\prime}\right)=\left(v^{\prime}+w\right) \perp v$. Since $v$ and $v^{\prime}$ are arbitrary, it follows that $\mathcal{T}^{\prime} \perp(\mathcal{T} \cap \mathcal{S})$. The non-degeneracy of $\pi_{\mathcal{T}}$ and $\pi_{\mathcal{T}}^{-1}$ yields $\operatorname{dim} \mathcal{T}^{\prime}=$ $q$.

Thus, we may regard the following mapping as the "inverse" projection $\pi_{\mathcal{T}}^{-1}$. To each $v \in \mathcal{S}$ it assigns the vector $w \in \operatorname{span}\{v, \mathcal{L}\} \cap \mathcal{T}$ such that $w=(v+\mathcal{L}) \cap \mathcal{T}$. It is well defined since $\mathcal{L} \perp \mathcal{S}$ and $\operatorname{dim}(\mathcal{T} \cap \mathcal{L})=0$.

(iv) Since $\mathcal{L} \cap \mathcal{N}$ is orthogonal to both $\mathcal{S}$ and $\mathcal{T}$, we have

$$
(v+\mathcal{L}) \cap \mathcal{T}=(v+\mathcal{E}) \cap \mathcal{T}
$$

for $v \in \mathcal{S}$, where $\mathcal{E} \subset(\mathcal{L} \cap \mathcal{N})^{\perp} \cap \mathcal{L}$ is the minimal subset enjoying this property. Let us prove that $\mathcal{E}=(\mathcal{L} \cap \mathcal{N})^{\perp} \cap \mathcal{L}$. It is easy to see that if 
$w=(v+\mathcal{L}) \cap \mathcal{T}=v+u$ with $u \in \mathcal{L}$ then $u=w-v \in \operatorname{span}\{\mathcal{S}, \mathcal{T}\}=(\mathcal{L} \cap \mathcal{N})^{\perp}$. Consequently, $u \in(\mathcal{L} \cap \mathcal{N})^{\perp} \cap \mathcal{L}$, and $\operatorname{dim} \mathcal{E} \leq N-\widetilde{N}-l$.

Verify that $\operatorname{dim} \mathcal{E} \geq N-\widetilde{N}-l$. Assume on the contrary that $\mathcal{E} \neq$ $(\mathcal{L} \cap \mathcal{N})^{\perp} \cap \mathcal{L}$, and consider $v_{0} \in \mathcal{E}^{\perp} \cap\left((\mathcal{L} \cap \mathcal{N})^{\perp} \cap \mathcal{L}\right)$. Since $v_{0} \in \operatorname{span}\{\mathcal{T}, \mathcal{S}\}=$ $(\mathcal{L} \cap \mathcal{N})^{\perp}$, it follows that $v_{0}=v_{0}^{\mathcal{T}}+v_{0}^{\mathcal{S}}$, where $v_{0}^{\mathcal{T}} \in \mathcal{T}$ and $v_{0}^{\mathcal{S}} \in \mathcal{S}$. Take $v=-v_{0}^{\mathcal{S}} \in \mathcal{S}$ and consider $v_{0}^{\mathcal{T}}=v+v_{0} \in \mathcal{T}$. By the assumption on $\mathcal{E}$ and because $\pi_{\mathcal{T}}$ is an epimorphism, $v+v_{0}=v^{\mathcal{S}}+v^{\mathcal{E}}$, where $v^{\mathcal{S}} \in \mathcal{S}$ and $v^{\mathcal{E}} \in \mathcal{E}$. Hence, $v-v^{\mathcal{S}}=v^{\mathcal{E}}-v_{0}$, where $v-v^{\mathcal{S}} \in \mathcal{S}$ and $v^{\mathcal{E}}-v_{0} \in \mathcal{L}$. Since $\mathcal{L} \perp \mathcal{S}$, we arrive at a contradiction.

(v) Similarly, we show that $\pi_{\mathcal{N}}^{-1}(w)=(w+\mathcal{F}) \cap \mathcal{N}$, where $\mathcal{F}=(\mathcal{S} \cap$ $\mathcal{T})^{\perp} \cap \mathcal{S}=\mathcal{S}^{\prime}$, and $\operatorname{dim} \mathcal{F}=\operatorname{dim} \mathcal{E}$.

(vi) Take a cube of radius $r$ in $\mathcal{S}$ which is the direct product of a cube of radius $r$ in $\mathcal{T} \cap \mathcal{S}$ and a cube of radius $r$ in $\mathcal{S}^{\prime}$. Then its image equals the direct product of a cube of radius $r$ in $\mathcal{T} \cap \mathcal{S}$ and a subset of $\mathcal{T}$. Hence, it suffices to calculate the measure distortion of $\pi_{\mathcal{T}}^{-1}$ on $\mathcal{S}^{\prime}$. Similarly, we infer that it suffices to calculate the measure distortion of $\pi_{\mathcal{N}}^{-1}$ on $\mathcal{L}^{\prime}$.

(vii) Consider the orthogonal mapping $\Theta$ that is a rotation about $\operatorname{span}\{\mathcal{N} \cap$ $\mathcal{L}, \mathcal{T} \cap \mathcal{S}\}$ such that

- $\Theta(\mathcal{F})=\mathcal{E}$

- $\Theta\left((\mathcal{N} \cap \mathcal{L})^{\perp} \cap \mathcal{L}\right)=(\mathcal{T} \cap \mathcal{S})^{\perp} \cap \mathcal{S}$.

Note that if we choose an orthonormal basis in $\mathbb{R}^{N}$ in the following order: $\operatorname{span}\{\mathcal{N} \cap \mathcal{L}, \mathcal{T} \cap \mathcal{S}\}, \operatorname{span}\{\mathcal{F}\}, \operatorname{span}\left\{(\mathcal{N} \cap \mathcal{L})^{\perp} \cap \mathcal{L}\right\}$ then the matrix of $\Theta$ looks like

$$
\Theta=\left(\begin{array}{ccc}
E & 0 & 0 \\
0 & 0 & E \\
0 & \widetilde{E} & 0
\end{array}\right),
$$

where

$$
\widetilde{E}=\left(\begin{array}{ccccc}
-1 & 0 & 0 & 0 & 0 \\
0 & 1 & 0 & 0 & 0 \\
0 & 0 & 1 & 0 & 0 \\
\cdot & \cdot & \cdot & \cdot & \cdot \\
0 & 0 & 0 & 0 & 1
\end{array}\right)
$$

Consequently, $\left.\pi_{\mathcal{T}}^{-1}\right|_{\mathcal{S}^{\prime}}\left(B(0, r) \cap \mathcal{S}^{\prime}\right)=\left.\Theta \circ \pi_{\mathcal{N}}^{-1}\right|_{\mathcal{L}^{\prime}}\left(B(0, r) \cap \mathcal{L}^{\prime}\right)$ for any $r>0$, and the measure distortions of these mappings coincide.

(viii) Thus, the measure distortions of $\pi_{\mathcal{N}}$ and $\pi_{\mathcal{T}}$ coincide.

Since both the Riemannian differential and $h c$-differential of $\theta_{x}$ at $x$ equal identity, by the chain rule the corresponding "determinants" coincide:

$$
\mathcal{D}(D \varphi(x))=\mathcal{D}(D \psi(0)) \text { and } \mathcal{D}(\widehat{D} \varphi(x))=\mathcal{D}(\widehat{D} \psi(0)) .
$$


Thus, the measure distortion under $\pi_{\mathcal{T}}^{-1}$ equals

$$
\frac{\mathcal{D}(D \psi(x))}{\mathcal{D}(\widehat{D} \psi(x))}=\frac{\mathcal{D}(D \varphi(x))}{\mathcal{D}(\widehat{D} \varphi(x))} .
$$

Step III. Verify that $\psi^{-1}(t) \cap \operatorname{Box}_{2}(0, r)$ is a subset of an $o(r)$-neighborhood of ker $\widehat{D} \psi(0) \cap \operatorname{Box}_{2}(0, r(1+o(1)))$ with respect to $d_{2 E}^{0}$. To this end, we prove that $\partial\left(\psi^{-1}(t) \cap \operatorname{Box}_{2}(0, r)\right)$ is a subset of the $o(r)$-neighborhood of $\partial\left(\right.$ ker $\left.\widehat{D} \psi(0) \cap \operatorname{Box}_{2}(0, r(1+o(1)))\right)$ with respect to $d_{2}$, where $o(1)$ is uniform in $x=\theta_{x}(0), x \in \mathcal{U} \Subset \mathbb{M}$.

Indeed, consider $y \in \partial\left(\psi^{-1}(t) \cap \operatorname{Box}_{2}(0, r)\right)$. We can represent this point as $y=y_{\widehat{D} \psi}+y_{\text {ker } \widehat{D} \psi}$, where $y_{\widehat{D} \psi} \in(\operatorname{ker} \widehat{D} \psi(0))^{\perp}$ and $y_{\text {ker } \widehat{D} \psi} \in \operatorname{ker} \widehat{D} \psi(0)$. Then the definition of the $h c$-differentiability implies that

$$
\begin{aligned}
o(r)=d_{2}^{\psi(0)}(\widehat{D} \psi(0)(y), \psi(y))=d_{2}^{\psi(0)}(\widehat{D} \psi(0) & \left.\left(y_{\widehat{D} \psi}\right), \psi(y)\right) \\
& =d_{2}^{\psi(0)}\left(\widehat{D} \psi(0)\left(y_{\widehat{D} \psi}\right), \psi(0)\right) .
\end{aligned}
$$

We have $d_{2}^{\psi(0)}\left(\psi(0), \widehat{D} \psi(0)\left(y_{\widehat{D} \psi}\right)\right)=o(r)$, and $d_{2}^{0}\left(0, y_{\widehat{D} \psi}\right)=o(r)$, where $o(\cdot)$ is uniform in $y, x=\theta_{x}(0), x \in \mathcal{U} \Subset \mathbb{M}$. The inequality

$$
d_{2 E}^{0}(0, u+v) \leq d_{2 E}^{0}(0, u)+c d_{2 E}^{0}(0, v)
$$

implies that

$$
d_{2 E}^{0}\left(0, y_{\text {ker } \widehat{D} \psi}\right) \leq d_{2 E}^{0}(0, y)+c d_{2 E}^{0}\left(0, y_{\widehat{D} \psi}\right)=r+o(r),
$$

and hence, $d_{2 E}^{0}\left(0, y_{\operatorname{ker} \widehat{D} \psi}\right)=r(1+o(1))$ and $d_{2}^{0}\left(0, y_{\operatorname{ker} \widehat{D} \psi}\right)=r(1+o(1))$. Thus, $\partial\left(\psi^{-1}(t) \cap \operatorname{Box}_{2}(0, r)\right)$ is a subset of the $o(r)$-neighborhood of $\partial(\operatorname{ker} \widehat{D} \psi(0) \cap$ $\left.\operatorname{Box}_{2}(0, r(1+o(1)))\right)$ with respect to $d_{2}$.

Step IV. Similarly considering the linear mapping $L(y)=D \psi(0) y$, we infer that $\partial\left(\operatorname{ker} D \psi(0) \cap \operatorname{Box}_{2}(0, r)\right)$ is a subset of the $o(r)$-neighborhood of $\partial\left(\right.$ ker $\left.\widehat{D} \psi(0) \cap \operatorname{Box}_{2}(0, r(1+o(1)))\right)$ with respect to $d_{2 E}^{0}$. Indeed, we have ker $D \psi(0)=L^{-1}(0)$ and $D L(0)=D \psi(0)$, and $L$ is $h c$-differentiable at 0 since $D \psi(0)$ is horizontal, and $\widehat{D} L(0)=\widehat{D} \psi(0)$.

Step V. Since there exists a bijective linear mapping from ker $\widehat{D} \psi(0) \cap$ $\operatorname{Box}_{2}(0, r)$ to $\operatorname{ker} D \psi(0) \cap \operatorname{Box}_{2}(0, r(1+o(1)))$, by Step IV we infer that $\partial\left(\operatorname{ker} \widehat{D} \psi(0) \cap \operatorname{Box}_{2}(0, r)\right)$ is a subset of the $o(r)$-neighborhood of $\partial($ ker $D \psi(0) \cap$ $\left.\operatorname{Box}_{2}(0, r(1+o(1)))\right)$.

Hence, $\partial\left(\psi^{-1}(t) \cap \operatorname{Box}_{2}(0, r)\right)$ is a subset of the $o(r)$-neighborhood of $\partial\left(\operatorname{ker} D \psi(0) \cap \operatorname{Box}_{2}(0, r(1+o(1)))\right)$ with respect to $d_{2 E}^{0}$. 
Step VI. In Steps VII - IX we explain that

$$
\partial\left(\operatorname{ker} D \psi(0) \cap \operatorname{Box}_{2}(0, r(1+o(1)))\right)
$$

is a subset of the $o(r)$-neighborhood of $\partial\left(\psi^{-1}(t) \cap \operatorname{Box}_{2}(0, r)\right)$ with respect to $d_{2 E}^{0}$. Moreover, similar arguments imply that the same is true regarding the sets ker $D \psi(0) \cap \operatorname{Box}_{2}(0, r(1+o(1)))$ and $\psi^{-1}(t) \cap \operatorname{Box}_{2}(0, r)$. Indeed, it suffices to recall that $o(\cdot)$ in the previous steps are uniform in $r>0$ and $x=\theta_{x}(0)$.

Step VII. In order to justify the result of Step VI, we construct a one-toone mapping from $\partial\left(\psi^{-1}(t) \cap \operatorname{Box}_{2}(x, r)\right)$ to a subset of the $o(r)$-neighborhood of $\partial\left(\operatorname{ker} D \psi(0) \cap \operatorname{Box}_{2}(x, r(1+o(1)))\right.$ ) (with respect to $d_{2 E}^{0}$ ) lying in the plane ker $D \psi(0)$.

First of all, observe that at the regular point 0 we have ker $D \psi \cap(\text { ker } \widehat{D} \psi)^{\perp}=$ $\{0\}$ by Step II, substep (iii).

From this we deduce the following assumption: without loss of generality we may assume that $D \psi(z)(v) \geq \beta$, where $z \in U \supset \operatorname{Box}_{2}(0, r), \beta>0$, for $v \in(\text { ker } \widehat{D} \psi(0))^{\perp}$ with $|v|=1$.

This implies that in a neighborhood of a regular point we can expand each point $y$ uniquely as $y=y_{\text {ker } D \psi}+y_{\widehat{D} \psi}$, where $y_{\operatorname{ker} D \psi} \in \operatorname{ker} D \psi$ and $y_{\widehat{D} \psi} \in(\operatorname{ker} \widehat{D} \psi)^{\perp}$. Suppose that $y \in \psi^{-1}(t) \cap \partial \operatorname{Box}_{2}(0, r)$ and consider the mapping $\xi: y \mapsto y_{\text {ker } D \psi}$.

Verify that $d_{2}(0, \xi(y))=r(1+o(1))$. To this end, we prove that $d_{2}^{0}(0, y-$ $\xi(y))=o(r)$ and then apply (3.9) for $d_{2 E}^{0}$.

Represent $y$ as $y=y_{\text {ker } D \psi}+y_{\widehat{D} \psi}=v_{\text {ker } \widehat{D} \psi}+v_{\widehat{D} \psi}+y_{\widehat{D} \psi}$, where $y_{\text {ker } D \psi}=$ $v_{\text {ker } \widehat{D} \psi}+v_{\widehat{D} \psi}$. Since $d_{2 E}^{0}(0, y)=r$, Step III implies that $d_{2 E}^{0}\left(0, v_{\operatorname{ker} \widehat{D} \psi}\right)=$ $r+o(r)$ and $d_{2 E}^{0}\left(0, v_{\widehat{D} \psi}+y_{\widehat{D} \psi}\right)=o(r)$. Moreover, by Step IV we have

$d_{2 E}^{0}\left(0, y_{\text {ker } D \psi}\right)=(1+o(1)) d_{2 E}^{0}\left(0, v_{\text {ker } \widehat{D} \psi}\right), \quad d_{2 E}^{0}\left(0, v_{D \psi}\right)=o(1) d_{2 E}^{0}\left(0, v_{\text {ker } \widehat{D} \psi}\right)$, and taking into account the generalized triangle inequality for $d_{2 E}^{0}$, we conclude that $d_{2 E}^{0}\left(0, y_{\widehat{D} \psi}\right)=o(r)$. Finally, we have $d_{2}^{0}(0, y-\xi(y))=o(r)$.

Step VIII. On this step, we show that $\xi$ is a bijective mapping. It is easy to see that $\xi$ is injective. Indeed, it follows from $D \psi\left\langle(\operatorname{ker} \widehat{D} \psi)^{\perp} \cap \mathbb{S}^{N-1}\right\rangle \geq$ $\beta>0$.

To verify that it is also surjective, we show that the mapping $\eta: \psi^{-1} \cap$ $\operatorname{Box}_{2}(0,2 r) \rightarrow \operatorname{ker} D \psi(0)$ is bi-Lipschitz. Here, $\eta$ assigns to each $y \in \psi^{-1}(t) \cap$ $\operatorname{Box}_{2}(0, r)$, the vector $y_{\text {ker } D \psi}$, where $y=y_{\text {ker } D \psi}+y_{\widehat{D} \psi}, y_{\text {ker } D \psi} \in \operatorname{ker} D \psi$, and $y_{\widehat{D} \psi} \in(\operatorname{ker} \widehat{D} \psi)^{\perp}$. It is clear that $\left.\eta\right|_{\psi^{-1}(t) \cap \partial \operatorname{Box}_{2}(0, r)}=\xi$.

Since $\eta$ is a projection, it is Lipschitz with the Lipschitz constant being equal to 1 . Verify that $\left|\eta\left(y_{1}\right)-\eta\left(y_{2}\right)\right| \geq K\left|y_{1}-y_{2}\right|$ for some $K>0$ for all $y_{1}, y_{2} \in \psi^{-1}(t) \cap \operatorname{Box}_{2}(0,2 r)$. 
Suppose that it is not so; thus, for every $\varepsilon>0$ there exist $y_{1}, y_{2} \in \psi^{-1}(t) \cap$ $\operatorname{Box}_{2}(0,2 r)$ such that $\left|\eta\left(y_{1}\right)-\eta\left(y_{2}\right)\right|<\varepsilon\left|y_{1}-y_{2}\right|$. Since $y_{i}=y_{i \operatorname{ker} D \psi}+y_{i \widehat{D} \psi}$, $y_{i_{\text {ker } D}} \in \operatorname{ker} D \psi$ and $y_{i_{\widehat{D}}} \in(\operatorname{ker} \widehat{D} \psi)^{\perp}, i=1,2$, we infer that

$$
\left|\eta\left(y_{1}\right)-\eta\left(y_{2}\right)\right|<\varepsilon\left|y_{1}-y_{2}\right| \leq \varepsilon\left|\eta\left(y_{1}\right)-\eta\left(y_{2}\right)\right|+\varepsilon\left|y_{1} \widehat{D} \psi-y_{2} \widehat{D} \psi\right|,
$$

and

$$
\left|y_{1 \widehat{D} \psi}-y_{2} \widehat{D} \psi\right| \geq \frac{1-\varepsilon}{\varepsilon}\left|\eta\left(y_{1}\right)-\eta\left(y_{2}\right)\right| .
$$

Since ker $D \psi(0) \cap(\operatorname{ker} \widehat{D} \psi(0))^{\perp}=0$, the norm $\left|y_{1}-y_{2}\right|_{\eta}=\left|\eta\left(y_{1}\right)-\eta\left(y_{2}\right)\right|+$ $\left|y_{1 \widehat{D} \psi}-y_{2} \widehat{D} \psi\right|$ is equivalent to the Euclidean norm. Furthermore,

$$
\psi\left(y_{1}\right)=\psi\left(y_{2}\right)+D \psi\left(y_{2}\right)\left(y_{1}-y_{2}\right)+o\left(\left|y_{1}-y_{2}\right|\right)
$$

and $D \psi\left(y_{2}\right)\left(y_{1}-y_{2}\right)=o\left(\left|y_{1}-y_{2}\right|\right)$. Since $\eta$ is a linear mapping, we have $y_{1} \widehat{D} \psi-y_{2} \widehat{D} \psi=\left(y_{1}-y_{2}\right)_{\widehat{D} \psi}$ and $\eta\left(y_{1}\right)-\eta\left(y_{2}\right)=\eta\left(y_{1}-y_{2}\right)$.

On the one hand, by the definition of $\eta$ we have

$$
\begin{aligned}
D \psi\left(y_{2}\right)\left(y_{1}-y_{2}\right)=D \psi(0)\left(y_{1}-y_{2}\right) & +o\left(\left|y_{1}-y_{2}\right|\right) \\
& =D \psi(0)\left(\left(y_{1}-y_{2}\right)_{\widehat{D} \psi}\right)+o\left(\left|y_{1}-y_{2}\right|\right),
\end{aligned}
$$

and $D \psi(0)\left(\left(y_{1}-y_{2}\right)_{\widehat{D} \psi}\right)=o\left(\left|y_{1}-y_{2}\right|\right)$. On the other hand,

$\left|y_{1} \widehat{D} \psi-y_{2} \widehat{D} \psi\right| \geq\left(1-\left(\frac{1-\varepsilon}{\varepsilon}+1\right)^{-1}\right)\left|y_{1}-y_{2}\right|_{\eta} \geq L\left(1-\left(\frac{1-\varepsilon}{\varepsilon}+1\right)^{-1}\right)\left|y_{1}-y_{2}\right|$,

where $L>0$ depends only on $U$.

These relations lead to a contradiction with

$$
D \psi(0)\left\langle(\operatorname{ker} \widehat{D} \psi(0))^{\perp}\right\rangle \geq \beta>0 .
$$

Thus, $\eta$ is a bi-Lipschitz mapping, and each $y \in \psi^{-1}(t) \cap \partial \operatorname{Box}_{2}(0, r)$ has a unique preimage. Thus, $\xi$ is bijective.

Step IX. Step VIII implies that $\xi$ is invertible, and the previous steps imply that the $d_{2}$-distortion of $\xi^{-1}$ is $(1+o(1))$ with respect to 0 . The estimate for $d_{2}\left(0, y-\eta^{-1}(y)\right)$ is proved in Step VII. Observe that $o(1)$ depends on the convergence of $o(1)$ to 0 in the equality

$$
d_{2}^{\psi(0)}(\widehat{D} \psi(0) y, \psi(y))=o(1) \cdot d_{2}(0, y) .
$$

Thus, $o(1)$ is uniform in $y$. Consequently, $\psi^{-1}(t) \cap \partial \operatorname{Box}_{2}(0, r)$ is a subset of the $o(r)$-neighborhood of ker $D \psi(0) \cap \partial \operatorname{Box}_{2}(0, r(1+o(1)))$ with respect to $d_{2}$, and conversely, ker $D \psi(0) \cap \partial \operatorname{Box}_{2}(0, r)$ is a subset of the $o(r)$-neighborhood 
of $\psi^{-1}(t) \cap \partial \operatorname{Box}_{2}(0, r)$ with respect to $d_{2 E}^{0}$ (see Step VII). The same is true regarding the sets $\psi^{-1}(t) \cap \operatorname{Box}_{2}(0, r)$ and ker $D \psi(0) \cap \operatorname{Box}_{2}(0, r(1+o(1)))$.

Note that the extension $\eta$ of $\xi$ onto $\psi^{-1}(t) \cap \operatorname{Box}_{2}(0, r)$ has the $d_{2}$-distortion with respect to 0 equal to $1+o(1)$ as well. In Step VIII, we prove that this extension is bijective.

Step X. On this step, we show that the classical, that is, "Riemannian" distortion of $\eta$ (and of $\xi$ ) is also $1+o(1)$.

Indeed, since ker $D \psi(0) \cap(\operatorname{ker} \widehat{D} \psi(0))^{\perp}=\{0\}$ and $D \psi\left\langle(\operatorname{ker} \widehat{D} \psi(0))^{\perp}\right\rangle \geq$ $\beta>0$ on $U$, it is easy to see that $\rho\left(0, y_{\widehat{D} \psi}\right)=o(\rho(0, y))$. Hence, $\rho\left(0, y_{\widehat{D} \psi}\right)=$ $o\left(\rho\left(0, y_{\text {ker } D \psi}\right)\right)$, and the $\rho$-distortion of both $\eta$ and $\eta^{-1}$ equals $1+o(1)$.

Since $o(\cdot)$ are uniform in the definitions of Riemannian differentiability and $h c$-differentiability, $o(\cdot)$ is uniform (both in the case of $d_{2^{-}}$and $\rho$ distortion).

Thus, claim $\mathbf{I}$ is proved.

Step XI. Using the results of all previous steps, we can consider the mapping $\eta^{-1}$ (that is, the extension of $\left.\xi^{-1}\right)$ from ker $D \psi \cap \operatorname{Box}_{2}(0, r(1+$ $o(1)))$ to $\psi^{-1}(t) \cap \operatorname{Box}_{2}(0, r)$ (see Steps VII-IX). The established properties of $\eta$ imply that the measure of $\psi^{-1}(t) \cap \operatorname{Box}_{2}(0, r)$ equals that of ker $D \psi \cap$ $\operatorname{Box}_{2}(0, r)$ up to a factor of $1+o(1)$, that is, it equals $C(1+o(1)) r^{\nu-\tilde{\nu}}$, where $C$ is obtained above.

Indeed, $\eta$ is a bi-Lipschitz $C^{1}$-mapping since it is a projection of a $C^{1}$ surface in a nontangent direction. Consequently, the inverse mapping $\eta^{-1}$ is also a bi-Lipschitz $C^{1}$-mapping. Moreover, since it is differentiable, it is also metrically differentiable, and its metric differential equals $1+o(1)$ on every direction. The $C^{1}$-mapping $\eta^{-1}$ satisfies $\mathcal{H}^{N-\widetilde{N}}\left(\eta^{-1}(A)\right)=\mathcal{J}\left(\eta^{-1}, 0\right)$. $\mathcal{H}^{N-\widetilde{N}}(A) \cdot(1+o(1))$, where $A=\eta\left(\psi^{-1}(t) \cap \operatorname{Box}_{2}(0, r)\right)$. We can calculate the Jacobian of $\eta^{-1}$ via its metric differential [Ki, Km1, Km4, Km3, Km5]:

$$
\mathcal{J}\left(\eta^{-1}, 0\right)=\sigma_{N-\widetilde{N}}\left[\int_{\mathbb{S}^{N-\widetilde{N}-1}}(1+o(1))^{\widetilde{N}-N} d \mathcal{H}^{N-\widetilde{N}}(u)\right]^{-1}=(1+o(1)) .
$$

Thus, $\mathcal{H}^{N-\widetilde{N}}\left(\eta^{-1}(A)\right)=\mathcal{H}^{N-\widetilde{N}}(A)(1+o(1))$. By taking into account a remark in Step $\mathbf{V}$ substep (ii) of the proof of Theorem 3.7 concerning regular points, we infer $\mathcal{H}^{N-\widetilde{N}}(A)=\prod_{k=1}^{M} \omega_{n_{k}-\tilde{n}_{k}} \cdot \frac{\mathcal{D}(D \varphi(x))}{\mathcal{D}(\widehat{D} \varphi(x))} r^{\nu-\tilde{\nu}}(1+o(1))$.

Step XII. Consider the restriction of $\theta_{x}$ to $\psi^{-1}(t) \cap \operatorname{Box}_{2}(0, r)$. Observe that $\theta_{x}\left(\psi^{-1}(t) \cap \operatorname{Box}_{2}(0, r)\right)=\varphi^{-1}(t) \cap \operatorname{Box}_{2}(x, r)$.

The $\mathcal{H}^{N-\widetilde{N}}$-measure distortion under this mapping equals $\widetilde{\mathcal{D}}\left(\left.g\right|_{\operatorname{ker} D \varphi(x)}\right)$, and $c_{\text {Riem }}=\widetilde{\mathcal{D}}\left(\left.g\right|_{\text {ker } D \varphi(x)}\right)$.

Claim II is proved. 
All $o(\cdot)$ in both claims $\mathbf{I}$ and $\mathbf{I I}$ are uniform in the radius $r$ and $x=\theta_{x}(0)$, $x \in \mathcal{U} \Subset \mathbb{M}$.

The proof of the theorem is complete.

Definition 3.12. The (spherical) Hausdorff measure of a set $A \subset \varphi^{-1}(t)$ (constructed with respect to a sub-Riemannian (quasi)metric $d$ and sub-Riemannian balls $B_{d}$ in $d$ ) equals

$$
\mathcal{H}^{\nu-\tilde{\nu}}(A)=\omega_{\nu-\tilde{\nu}} \liminf _{\delta \rightarrow 0}\left\{\sum_{i \in \mathbb{N}} r_{i}^{\nu-\tilde{\nu}}: \bigcup_{i \in \mathbb{N}} B_{d}\left(x_{i}, r_{i}\right) \supset A, x_{i} \in A, r_{i} \leq \delta, i \in \mathbb{N}\right\} .
$$

Property 3.13 ([KmVod $)$. The quasimetric $d_{2}$ and the metric $d_{c c}$ (see Definitions 2.9 and 2.11) are locally equivalent.

Lemma 3.14. Given a set $A \subset \varphi^{-1}(t)$ of $\mathcal{H}^{N-\widetilde{N}}$-measure zero consisting of regular points and $\varepsilon>0$, there exists a covering of $A$ by the "balls" $\left\{\operatorname{Box}_{2}\left(x_{j}, r_{j}\right) \cap \varphi^{-1}(t)\right\}_{j \in \mathbb{N}}$, where $x_{j} \in \varphi^{-1}(t), j \in \mathbb{N}$, the sum of whose $\mathcal{H}^{N-\widetilde{N}}$-measures is less than $\varepsilon$.

Proof. Fix $\varepsilon>0$. Represent $A$ as the union of some subsets $A_{l} \subset A, l \in \mathbb{N}$, lying at positive distances from the set $\chi \cap \varphi^{-1}(t)$. Without loss of generality we consider a set $A_{l}$ instead of $A$. Suppose that $A_{l}$ satisfies the stated conditions. Observe that for this set there exists a collection of "balls" $\left\{B\left(x_{j}, r_{j}\right) \cap \varphi^{-1}(t)\right\}_{j \in \mathbb{N}}$, where $x_{j} \in \varphi^{-1}(t)$ and $B\left(x_{j}, r_{j}\right)$ are Riemannian balls, $j \in \mathbb{N}$, the sum of whose $\mathcal{H}^{N-\widetilde{N}}$-measures is less than $\tilde{\varepsilon}$, where $\tilde{\varepsilon}$ is determined by $\varepsilon$ (we specify the exact expression for $\tilde{\varepsilon}$ below). Fix $j \in \mathbb{N}$. For $B\left(x_{j}, r_{j}\right) \cap \varphi^{-1}(t)$, there exists a collection

$$
\left\{B_{c c}(x, r) \cap \varphi^{-1}(t): x \in A_{l} \cap \varphi^{-1}(t), B_{c c}(x, r) \cap \varphi^{-1}(t) \subset B\left(x_{j}, r_{j}\right), r>0\right\} .
$$

By the $5 r$-covering lemma (see [Fe2] for instance) there exists a countable family of disjoint "balls" $\left\{B_{c c}\left(x_{j_{k}}, r_{j_{k}}\right) \cap \varphi^{-1}(t)\right\}$ such that

$$
\bigcup_{k \in \mathbb{N}} B_{c c}\left(x_{j_{k}}, 5 r_{j_{k}}\right) \supset A_{l} \cap B\left(x_{j}, r_{j}\right) .
$$

(Here we use the metric $d_{c c}$ since the $5 r$-covering lemma is established for metrics, and we cannot say for sure whether it holds for quasimetrics). Since $d_{2}$ and $d_{c c}$ are locally equivalent, there exist some constants $0<C_{1}, C_{2}<\infty$ such that $\operatorname{Box}_{2}\left(x, C_{1} r\right) \subset B_{c c}(x, r) \subset \operatorname{Box}_{2}\left(x, C_{2} r\right)$ for sufficiently small $r>0$ and $x$ in some sufficiently small neighborhood. Consequently, there exists a disjoint collection $\left\{\operatorname{Box}_{2}\left(x_{j_{k}}, C_{1} r_{j_{k}}\right)\right\}$ such that

$$
\bigcup_{k \in \mathbb{N}} \operatorname{Box}_{2}\left(x_{j_{k}}, 5 C_{2} r_{j_{k}}\right) \supset A_{l} \cap B\left(x_{j}, r_{j}\right) .
$$


This implies that

$$
\begin{aligned}
\sum_{k \in \mathbb{N}} \mathcal{H}^{N-\widetilde{N}}\left(\operatorname{Box}_{2}\left(x_{j_{k}}, 5 C_{2} r_{j_{k}}\right)\right) \leq & C\left(5, C_{1}, C_{2}\right) \sum_{k \in \mathbb{N}} \mathcal{H}^{N-\widetilde{N}}\left(\operatorname{Box}_{2}\left(x_{j_{k}}, C_{1} r_{j_{k}}\right)\right) \\
& \leq C\left(5, C_{1}, C_{2}\right) \mathcal{H}^{N-\widetilde{N}}\left(B\left(x_{j}, r_{j}\right) \cap \varphi^{-1}(t)\right)
\end{aligned}
$$

Then $\left\{\operatorname{Box}_{2}\left(x_{j_{k}}, 5 C_{2} r_{j_{k}}\right)\right\}_{j, k \in \mathbb{N}}$ is a reqired collection, and the sum of the $\mathcal{H}^{N-\widetilde{N}}$-measures of these "balls" is at most $C\left(5, C_{1}, C_{2}\right) \tilde{\varepsilon}<\varepsilon$.

Corollary 3.15. For each $\mathcal{H}^{N-\widetilde{N}}$-measure zero subset of $\varphi^{-1}(t)$ consisting of regular points we have $\mathcal{H}^{\nu-\tilde{\nu}}=0$.

The proof follows directly from the definition of an $\mathcal{H}^{\nu-\tilde{\nu}}$-negligible set.

Lemma 3.16. Consider a regular point $x \in \mathbb{M}$. Then given a sufficiently small $r>0$ with $\operatorname{Box}_{2}(x, r) \cap \varphi^{-1}(\varphi(x)) \cap \chi=\varnothing$ and $\varepsilon>0$, there exists a covering of $\operatorname{Box}_{2}(x, r) \cap \varphi^{-1}(\varphi(x))$ by the sets $\operatorname{Box}_{2}\left(x_{i}, r_{i}\right) \cap \varphi^{-1}(\varphi(x))$ with $x_{i} \in \varphi^{-1}(\varphi(x))$ and $\operatorname{Box}_{2}\left(x_{i}, r_{i}\right) \subset \operatorname{Box}_{2}(x, r), i \in \mathbb{N}$, such that

$$
\sum_{i \in \mathbb{N}} \mathcal{H}^{N-\widetilde{N}}\left(\operatorname{Box}_{2}\left(x_{i}, r_{i}\right) \cap \varphi^{-1}(\varphi(x))\right)<\mathcal{H}^{N-\widetilde{N}}\left(\operatorname{Box}_{2}(x, r) \cap \varphi^{-1}(\varphi(x))\right)+\varepsilon .
$$

Proof. Without loss of generality we assume that $r>0$ satisfies

$$
\operatorname{dist}\left(\operatorname{Box}_{2}(x, r) \cap \varphi^{-1}(\varphi(x)), \chi\right)>0 .
$$

Since the measure $\mathcal{H}^{N-\widetilde{N}}$ is doubling on $\operatorname{Box}_{2}(y, s) \cap \varphi^{-1}(\varphi(x))$ for $y \in$ $\varphi^{-1}(\varphi(x))$ and sufficiently small $s$, in view of the Vitali covering theorem there exists a collection of "balls" $\left\{\operatorname{Box}_{2}\left(x_{i_{j}}, r_{i_{j}}\right) \cap \varphi^{-1}(\varphi(x))\right\}_{j \in \mathbb{N}}$ with $x_{i_{j}} \in$ $\varphi^{-1}(\varphi(x))$ and $\operatorname{Box}_{2}\left(x_{i_{j}}, r_{i_{j}}\right) \cap \varphi^{-1}(\varphi(x)) \subset \operatorname{Box}_{2}(x, r) \cap \varphi^{-1}(\varphi(x)), j \in \mathbb{N}$, such that

$$
\mathcal{H}^{N-\widetilde{N}}\left(\operatorname{Box}_{2}(x, r) \cap \varphi^{-1}(\varphi(x))\right)=\sum_{j \in \mathbb{N}} \mathcal{H}^{N-\widetilde{N}}\left(\operatorname{Box}_{2}\left(x_{i_{j}}, r_{i_{j}}\right) \cap \varphi^{-1}(\varphi(x))\right) .
$$

By Lemma 3.14, the $\mathcal{H}^{N-\widetilde{N}}$-negligible set

$$
\operatorname{Box}_{2}(x, r) \cap \varphi^{-1}(\varphi(x)) \backslash \bigcup_{j \in \mathbb{N}} \operatorname{Box}_{2}\left(x_{i_{j}}, r_{i_{j}}\right) \cap \varphi^{-1}(\varphi(x)),
$$

admits a covering by the collection $\left\{\operatorname{Box}_{2}\left(x_{i_{k}}, r_{i_{k}}\right) \cap \varphi^{-1}(\varphi(x))\right\}$ of "balls" the sum of whose $\mathcal{H}^{N-\widetilde{N}}$-measures is less than the given $\varepsilon>0$. The proof is complete. 
Recall that $D_{\mu_{1}} \mu_{2}(y)$ stands for the derivative of a measure $\mu_{2}$ with respect to a measure $\mu_{1}$ at $y$ :

$$
D_{\mu_{1}} \mu_{2}(y)=\lim _{r \rightarrow 0} \frac{\mu_{2}(B(y, r))}{\mu_{1}(B(y, r))}
$$

Theorem 3.17 (The Lebesgue Differentiation of Measures on Level Sets). The Hausdorff measure $\mathcal{H}^{\nu-\tilde{\nu}}$ of $\operatorname{Box}_{2}(x, r) \cap \varphi^{-1}(\varphi(x))$, where $x$ is a regular point, and $\operatorname{dist}\left(\operatorname{Box}_{2}(x, r) \cap \varphi^{-1}(\varphi(x)), \chi\right)>0$, asymptotically equals $\omega_{\nu-\tilde{\nu}} r^{\nu-\tilde{\nu}}$ :

$$
\mathcal{H}^{\nu-\tilde{\nu}}\left(\operatorname{Box}_{2}(x, r) \cap \varphi^{-1}(\varphi(x))\right)=\omega_{\nu-\tilde{\nu}} r^{\nu-\tilde{\nu}}(1+o(1))
$$

The derivative $D_{\mathcal{H}^{N-\tilde{N}}} \mathcal{H}^{\nu-\tilde{\nu}}(x)$ equals

$$
\frac{1}{\widetilde{\mathcal{D}}\left(\left.g\right|_{\operatorname{ker} D \varphi(x)}\right)} \cdot \frac{\omega_{\nu-\tilde{\nu}}}{\prod_{k=1}^{M} \omega_{n_{k}-\tilde{n}_{k}}} \cdot \frac{\mathcal{D}(\widehat{D} \varphi(x))}{\mathcal{D}(D \varphi(x))} .
$$

Proof. Consider the intersection of the sub-Riemannian ball of a sufficiently small radius $r>0$ centered at $x$ and the level set $\varphi^{-1}(\varphi(x))$. Fix $\delta>0$ and some covering $\left\{\operatorname{Box}_{2}\left(y_{i}, r_{i}\right)\right\}_{i \in \mathbb{N}}$ of this intersection by sub-Riemannian balls as in the definition of the set function $\mathcal{H}_{\delta}^{\nu-\tilde{\nu}}$. Recall that for a (quasi)metric $d$ we have

$$
\mathcal{H}_{\delta}^{\nu-\tilde{\nu}}(A)=\omega_{\nu-\tilde{\nu}} \inf \left\{\sum_{i \in \mathbb{N}} r_{i}^{\nu-\tilde{\nu}}: \bigcup_{i \in \mathbb{N}} B_{d}\left(x_{i}, r_{i}\right) \supset A, x_{i} \in A, r_{i} \leq \delta, i \in \mathbb{N}\right\}
$$

Then, setting

$$
\alpha(y)=\lim _{r \rightarrow 0} \frac{\omega_{\nu-\tilde{\nu}} r^{\nu-\tilde{\nu}}}{\mathcal{H}^{N-\widetilde{N}}\left(\operatorname{Box}_{2}(y, r) \cap \varphi^{-1}(\varphi(y))\right)},
$$

by Theorem 3.11, we have

$$
\begin{aligned}
\omega_{\nu-\tilde{\nu}} \sum_{i \in \mathbb{N}} r_{i}^{\nu-\tilde{\nu}} & =\sum_{i \in \mathbb{N}}\left[\alpha\left(y_{i}\right)+\delta\left(y_{i}, r_{i}\right)\right] \mathcal{H}^{N-\widetilde{N}}\left(\operatorname{Box}_{2}\left(y_{i}, r_{i}\right) \cap \varphi^{-1}(\varphi(x))\right) \\
& =[\alpha(x)+\Delta(x, r)] \sum_{i \in \mathbb{N}} \mathcal{H}^{N-\widetilde{N}}\left(\operatorname{Box}_{2}\left(y_{i}, r_{i}\right) \cap \varphi^{-1}(\varphi(x))\right),
\end{aligned}
$$

where $\delta\left(y_{i}, r_{i}\right) \rightarrow 0$ as $r_{i} \rightarrow 0$ uniformly in $i \in \mathbb{N}$, and $\Delta(x, r) \rightarrow 0$ as $r \rightarrow 0$, since $g, D \varphi$ and $\widehat{D} \varphi$ are continuous, and by Theorem 3.11 as well. For the 
fixed $x$ and $\varepsilon>0$, we can choose a sufficiently small radius $r>0$ to satisfy $|\Delta(x, r)|<\varepsilon \cdot \alpha(x)$. Consequently,

$$
\begin{aligned}
& (1-\varepsilon) \alpha(x) \sum_{i \in \mathbb{N}} \mathcal{H}^{N-\widetilde{N}}\left(\operatorname{Box}_{2}\left(y_{i}, r_{i}\right) \cap \varphi^{-1}(\varphi(x))\right) \\
& \quad \leq \omega_{\nu-\tilde{\nu}} \sum_{i \in \mathbb{N}} r_{i}^{\nu-\tilde{\nu}} \leq(1+\varepsilon) \alpha(x) \sum_{i \in \mathbb{N}} \mathcal{H}^{N-\widetilde{N}}\left(\operatorname{Box}_{2}\left(y_{i}, r_{i}\right) \cap \varphi^{-1}(\varphi(x))\right) .
\end{aligned}
$$

Note that for each $\delta>0$ if the covering in the definition of $\mathcal{H}_{\delta}^{\nu-\tilde{\nu}}$ is nearly "optimal" (i. e., $\sum_{i \in \mathbb{N}} r_{i}^{\nu-\tilde{\nu}}$ is nearly minimal), then $\sum_{i \in \mathbb{N}} \mathcal{H}^{N-\widetilde{N}}\left(\operatorname{Box}_{2}\left(y_{i}, r_{i}\right) \cap\right.$ $\left.\varphi^{-1}(\varphi(x))\right)$ is also nearly minimal. Since the $\mathcal{H}^{N-\widetilde{N}}$-measure is countably additive, by Lemma 3.16 the infimum of the values of the last sum equals $\mathcal{H}^{N-N}\left(\operatorname{Box}_{2}(x, r)\right)$.

Hence,

$$
\mathcal{H}^{\nu-\tilde{\nu}}\left(\operatorname{Box}_{2}(x, r) \cap \varphi^{-1}(\varphi(x))\right)=\omega_{\nu-\tilde{\nu}} r^{\nu-\tilde{\nu}}(1+o(1))
$$

where $o(1) \rightarrow 0$ as $r \rightarrow 0$, and

$$
\begin{aligned}
D_{\mathcal{H}^{N-\tilde{N}}} \mathcal{H}^{\nu-\tilde{\nu}}(x) & =\alpha(x)=\lim _{r \rightarrow 0} \frac{\omega_{\nu-\tilde{\nu}} r^{\nu-\tilde{\nu}}}{\mathcal{H}^{N-\widetilde{N}}\left(\operatorname{Box}_{2}(x, r) \cap \varphi^{-1}(\varphi(x))\right)} \\
& =\frac{1}{\widetilde{\mathcal{D}}\left(\left.g\right|_{\operatorname{ker} D \varphi(x)}\right)} \cdot \frac{\omega_{\nu-\tilde{\nu}}}{\prod_{k=1}^{M} \omega_{n_{k}-\tilde{n}_{k}}} \cdot \frac{\mathcal{D}(\widehat{D} \varphi(x))}{\mathcal{D}(D \varphi(x))} .
\end{aligned}
$$

The proof is complete.

The last result motivates the next definition.

Definition 3.18. The sub-Riemannian coarea factor equals

$$
\mathcal{J}_{\widetilde{N}}^{S R}(\varphi, x)=\mathcal{D}(\widehat{D} \varphi(x)) \cdot \frac{\omega_{N}}{\omega_{\nu}} \frac{\omega_{\tilde{\nu}}}{\omega_{\widetilde{N}}} \frac{\omega_{\nu-\tilde{\nu}}}{\prod_{k=1}^{M} \omega_{n_{k}-\tilde{n}_{k}}} .
$$

Remark 3.19. By (3.10), we have

$$
\mathcal{J}_{\widetilde{N}}^{S R}(\varphi, x)=\mathcal{J}_{\widetilde{N}}(\varphi, x) \cdot D_{\mathcal{H}^{N-\tilde{N}}} \mathcal{H}^{\nu-\tilde{\nu}}(x) \cdot \frac{\widetilde{\mathcal{D}}(g(x))}{\widetilde{\mathcal{D}}(\tilde{g}(\varphi(x)))} \cdot \frac{\omega_{N}}{\omega_{\nu}} \frac{\omega_{\tilde{\nu}}}{\omega_{\tilde{N}}}
$$

since

$$
\mathcal{J}_{\widetilde{N}}(\varphi, x)=\mathcal{D}(D \varphi(x)) \cdot \frac{\widetilde{\mathcal{D}}(\tilde{g}(\varphi(x))) \widetilde{\mathcal{D}}\left(\left.g\right|_{\text {ker } D \varphi(x)}\right)}{\widetilde{\mathcal{D}}(g(x))}
$$


Corollary 3.20. Theorem 3.17, Corollary [3.15, and the Lebesgue differentiation theorem (see, for instance, [VodU]) imply that every measurable subset of a regular set $A \subset\left(\mathbb{D} \cap \varphi^{-1}(t)\right), t \in \widetilde{\mathbb{M}}$, satisfies

$$
\mathcal{H}^{\nu-\tilde{\nu}}(A)=\int_{A} D_{\mathcal{H}^{N-\widetilde{N}}} \mathcal{H}^{\nu-\tilde{\nu}}(x) d \mathcal{H}^{N-\widetilde{N}}(x)
$$

Theorem 3.21 (Local coarea formula for the set $\mathbb{D}$ ). If $\varphi \in C^{1}(\mathbb{M}, \widetilde{\mathbb{M}})$ then

$$
\int_{\mathbb{D}} \mathcal{J}_{\widetilde{N}}^{S R}(\varphi, x) d \mathcal{H}^{\nu}(x)=\int_{\widetilde{\mathbb{M}}} d \mathcal{H}^{\tilde{\nu}}(t) \int_{\varphi^{-1}(t) \cap \mathbb{D}} d \mathcal{H}^{\nu-\tilde{\nu}}(u) .
$$

Proof. The Riemannian coarea formula [Fe2] yields

$$
\begin{aligned}
\int_{\mathbb{D}} \mathcal{J}_{\widetilde{N}}^{S R}(\varphi, x) d \mathcal{H}^{\nu}(x) & =\int_{\mathbb{D}} \frac{\mathcal{J}_{\widetilde{N}}^{S R}(\varphi, x)}{\mathcal{D}(g(x))} d \mathcal{H}^{N}(x) \\
& =\int_{\widetilde{\mathbb{M}}} d \mathcal{H}^{\widetilde{N}}(t) \int_{\varphi^{-1}(t) \cap \mathbb{D}} \frac{\mathcal{J}_{\widetilde{N}}^{S R}(\varphi, u)}{\mathcal{J}_{\widetilde{N}}(\varphi, u) \widetilde{\mathcal{D}}(g(u))} d \mathcal{H}^{N-\widetilde{N}}(u) .
\end{aligned}
$$

Calculating the measure derivatives in $\widetilde{\mathbb{M}}$, we obtain

$$
\begin{aligned}
\int_{\widetilde{\mathbb{M}}} d \mathcal{H}^{\widetilde{N}}(t) \int_{\varphi^{-1}(t) \cap \mathbb{D}} \frac{\mathcal{J}_{\widetilde{N}}^{S R}(\varphi, u)}{\mathcal{J}_{\widetilde{N}}(\varphi, u) \widetilde{\mathcal{D}}(g(u))} d \mathcal{H}^{N-\widetilde{N}}(u) \\
=\int_{\widetilde{\mathbb{M}}} d \mathcal{H}^{\tilde{\nu}}(t) \int_{\varphi^{-1}(t) \cap \mathbb{D}} \frac{\mathcal{J}_{\widetilde{N}}^{S R}(\varphi, u) \widetilde{\mathcal{D}}(\tilde{g}(t))}{\mathcal{J}_{\widetilde{N}}(\varphi, u) \widetilde{\mathcal{D}}(g(u))} d \mathcal{H}^{N-\widetilde{N}}(u) .
\end{aligned}
$$

Finally, taking Remark 3.19 and Corollary 3.20 into account, we infer that

$$
\begin{aligned}
\int_{\widetilde{\mathbb{M}}} d \mathcal{H}^{\tilde{\nu}}(t) \int_{\varphi^{-1}(t) \cap \mathbb{D}} \frac{\mathcal{J}_{\widetilde{N}}^{S R}(\varphi, u) \widetilde{\mathcal{D}}(\tilde{g}(t))}{\mathcal{J}_{\widetilde{N}}(\varphi, u) \widetilde{\mathcal{D}}(g(u))} d \mathcal{H}^{N-\widetilde{N}}(u) \\
\quad=\int_{\widetilde{\mathbb{M}}} d \mathcal{H}^{\tilde{\nu}}(t) \int_{\varphi^{-1}(t) \cap \mathbb{D}} d \mathcal{H}^{\nu-\tilde{\nu}}(u) .
\end{aligned}
$$

The proof is complete. 


\section{The Characteristic Set}

The goal of this section is to prove

Theorem 4.1 (see also [Km7]). If Assumption [2.26 is fulfilled then

$$
\mathcal{H}^{\nu-\tilde{\nu}}\left(\chi_{t}\right)=0
$$

for almost all $t \in \widetilde{\mathbb{M}}$ (with respect to both $\mathcal{H}^{\widetilde{N}}$ and $\left.\mathcal{H}^{\tilde{\nu}}\right)$.

Recall that we denote $\varphi^{-1}(t) \cap \chi$ by $\chi_{t}$ for $t \in \widetilde{\mathbb{M}}$.

We prove the results of this section under the following assumptions.

Assumption 4.2. Assume that the basis vector fields $\left\{X_{i}\right\}_{i=1}^{N}$ in the preimage are of class $C^{M+1}$, and $\left\{\widetilde{X}_{j}\right\}_{j=1}^{\widetilde{N}}$ in the image are of class $C^{1, \tilde{\alpha}}, \tilde{\alpha}>0$. Moreover, assume that $\varphi \in C^{M+2}(\mathbb{M}, \widetilde{\mathbb{M}})$, where $M$ is the depth of $\mathbb{M}$ (see Definition 2.1).

Lemma 4.3. Let $\mathbb{M}$ be a Carnot manifold with $C^{1, \alpha}$-smooth basis vector fields, $\alpha>0$. From the basis vector fields $\left\{X_{i}\right\}_{i=1}^{N}$ construct linearly independent vector fields $\left\{Y_{j}\right\}_{j=1}^{N}$ as $Y_{j}(x)=\sum_{i=1}^{\operatorname{dim} H_{\operatorname{deg} X_{j}}} a_{j i}(x) X_{i}$, where $a_{j i}(x)$ are $C^{1, \omega}$-smooth functions for all $i$ and $j, \omega>0$. Then the quasimetric of type $d_{2}$ constructed with respect to the new basis $\left\{Y_{j}\right\}_{j=1}^{N}$ is locally equivalent to the initial quasimetric $d_{2}$.

Proof. Denote the quasimetric of type $d_{2}$ and the Carnot-Carathéodory metric constructed with respect to the basis $\left\{Y_{j}\right\}_{j=1}^{N}$ by $d_{2}^{Y}$ and $d_{c c}^{Y}$ respectively. The Ball-Box Theorem of [KmVod, Km9 implies that $d_{2}$ and $d_{c c}$ are locally equivalent. Moreover, since the lengths of a curve calculated with respect to different bases are bi-Lipschitz equivalent, the metrics $d_{2}^{Y}$ and $d_{c c}^{Y}$ are bi-Lipschitz equivalent as well. Considering that $d_{c c}$ and $d_{c c}^{Y}$ are locally equivalent, we infer that so are $d_{2}$ and $d_{2}^{Y}$.

Proof of Theorem 4.1. Observe that the condition $\mathcal{H}^{N}(\chi)=\mathcal{H}^{\nu}(\chi)=0$ and [Fe2, 2.10.25] immediately imply the result.

Assume now that $\mathcal{H}^{N}(\chi)>0$.

Step I. Divide $\chi$ into finitely many sets, each of which is defined by the structure of ker $D \varphi$. In other words, represent $\chi$ as

$$
\chi=\bigcup_{p_{1}, \ldots, p_{M}} \chi_{p_{1} \ldots p_{M}}
$$


where

$$
\chi_{p_{1} \ldots p_{M}}=\left\{x \in \chi: \operatorname{dim}\left(H_{i}(x) \cap \operatorname{ker} D \varphi(x)\right)=p_{i}, i=1, \ldots, M\right\} .
$$

The assumption $\mathcal{H}^{N}(\chi)>0$ implies the existence of a collection $\left\{p_{1}, \ldots, p_{M}\right\}$ with $\mathcal{H}^{N}\left(\chi_{p_{1} \ldots p_{M}}\right)>0$ since $\mathcal{H}^{N}(\chi) \leq \sum_{p_{1}, \ldots, p_{M}} \mathcal{H}^{N}\left(\chi_{p_{1} \ldots p_{M}}\right)$.

Step II. Fix a collection $\left\{p_{1}, \ldots, p_{M}\right\}$ with this property, the corresponding set $\chi_{p_{1} \ldots p_{M}}$, and $x \in \chi_{p_{1} \ldots p_{M}}$ (which is not necessarily a density point). The purpose of Steps II-V is to prove that

- the set $\chi_{p_{1} \ldots p_{M}}$ is measurable;

- in the coordinates of the first kind the order of tangency between a level set and its tangent plane is $o\left(r^{M}\right)$ at almost every $x \in \chi_{p_{1} \ldots p_{M}}$ (with respect to both $\mathcal{H}^{N}$ and $\mathcal{H}^{\nu}$ ),

these facts that are independent of the basis transformations described in Lemma 4.3 on sufficiently small $\mathcal{U} \Subset \mathbb{M}$. Thus, without loss of generality we may assume that at this chosen point $x$ the differential $D \varphi(x)$ is degenerate on basis vector fields $X_{i_{1}}(x), \ldots, X_{i_{N-\widetilde{N}}}(x)$. Consider a compact neighborhood $U \Subset \mathbb{M}$ at whose points $y$ the differential $D \varphi(y)$ does not vanish on the same vector fields as at $x$.

Step III. On Step IV, we are going to prove that $\mathcal{H}^{N}\left(\chi_{p_{1} \ldots p_{M}}\right)$ is measurable. To this end, we now construct a new auxiliary basis $\left\{Y_{j}\right\}_{j=1}^{N}$ in the same way as in Lemma 4.3. This construction consists of several steps.

(i) The purpose of this substep is to obtain a new horizontal basis the nonzero images of whose vectors under $D \varphi$ at $\chi_{p_{1} \ldots p_{M}}$ are independent.

The definition of $\chi_{p_{1} \ldots p_{M}}$ and the structures of $D \varphi$ and $\widehat{D} \varphi$ (see (2.5) and (2.4) ) imply that

$$
\operatorname{dim}\left(\operatorname{ker} \widehat{D} \varphi(x) \cap H_{1}(x)\right)=\operatorname{dim}\left(\operatorname{ker} D \varphi(x) \cap H_{1}(x)\right)=p_{1},
$$

where $p_{1}>n_{1}-\tilde{n}_{1}$ (see Proposition 2.21, claim I (a)). Then, the images of the remaining $n_{1}-p_{1}$ horizontal vectors are linearly independent on $\varphi(U)$.

Next, we consider the matrix of the classical differential $D \varphi$ written with respect to the basis vector fields $X_{1}, \ldots, X_{N}$ in the preimage and $\widetilde{X}_{1}, \ldots, \widetilde{X}_{\widetilde{N}}$ in the image.

Consider the $\tilde{n}_{1} \times n_{1}$ block of the matrix of $D \varphi$ that corresponds to the horizontal subspaces. Identify its $n_{1}$ columns as vectors in $\mathbb{R}^{\tilde{n}_{1}}$. Then, at $x$ we choose from this collection $n_{1}-p_{1}$ linearly independent vectors $w_{1}(x), \ldots, w_{n_{1}-p_{1}}(x)$. At a point $y \in U$ we denote the corresponding column vector as $w_{1}(y), \ldots, w_{n_{1}-p_{1}}(y)$. The remaining $p_{1}$ vectors $v_{1}(y), \ldots, v_{p_{1}}(y)$ 
at $y \in \chi_{p_{1} \ldots p_{M_{1}}} \cap U$ are contained in the linear span of the first ones. Consequently, for the set $\chi_{p_{1} \ldots p_{M}}$ there exists a transformation $O_{n_{1}-p_{1}}^{\tilde{n}_{1}}(y)$ of $\mathbb{R}^{\tilde{n}_{1}}$ depending $C^{M+1}$-smoothly on $y \in U$ such that the images of all $w_{1}(y), \ldots, w_{n_{1}-p_{1}}(y)$ belong to $\mathbb{R}^{n_{1}-p_{1}} \times 0^{\tilde{n}_{1}-\left(n_{1}-p_{1}\right)}$. To see that this mapping is $C^{M+1}$-smooth, it suffices to write the coordinates of the linearly independent vectors $\left\{w_{1}(y), \ldots, w_{n_{1}-p_{1}}(y), e_{i_{1}}, \ldots, e_{{\tilde{n_{1}}}_{-}\left(n_{1}-p_{1}\right)}\right\}$ in the standard basis as a matrix and then to calculate the inverse matrix. (Here $\left\{e_{i_{1}}, \ldots, e_{i_{\tilde{n}_{1}-\left(n_{1}-p_{1}\right)}}\right\}$ are the vectors of the standard basis in $\mathbb{R}^{\tilde{n}_{1}}$ linearly independent together with $w_{1}(x), \ldots, w_{n_{1}-p_{1}}(x)$; without loss of generality we may assume that the neighborhood $U$ is sufficiently small for the vectors $\left\{e_{i_{1}}, \ldots, e_{i_{\tilde{n}_{1}-\left(n_{1}-p_{1}\right)}}\right\}$ to be independent together with $w_{1}(x), \ldots, w_{n_{1}-p_{1}}(x)$ for all $y \in U$.) The resulting matrix is the matrix of this transformation. In other words, the matrix

$$
\left(O_{n_{1}-p_{1}}^{\tilde{n}_{1}}(y)\left[w_{1}(y)\right], \ldots, O_{n_{1}-p_{1}}^{\tilde{n}_{1}}(y)\left[w_{n_{1}-p_{1}}(y)\right]\right)
$$

has exactly $\tilde{n}_{1}-\left(n_{1}-p_{1}\right)$ vanishing columns. Since we can apply to these vectors an orthogonal transformation independent of $y \in U$ without loss of generality we may assume that these vanishing columns have indices from $n_{1}-p_{1}+1$ to $\tilde{n}_{1}$.

By the linear dependence, at the points of $\chi_{p_{1} \ldots p_{M}}$ the images of the column vectors $v_{1}(y), \ldots, v_{p_{1}}(y)$ also belong to $\mathbb{R}^{n_{1}-p_{1}}$. Represent these images (at an arbitrary point $y \in U$ ) as

$$
O_{n_{1}-p_{1}}^{\tilde{n}_{1}}(y)\left[v_{k}(y)\right]=\left(\omega_{k}^{1}(y), \omega_{k}^{2}(y)\right)^{T}
$$

where the dimension of $\omega_{k}^{1}(y)$ equals $n_{1}-p_{1}$, and the dimension of $\omega_{k}^{2}(y)$ equals $\tilde{n}_{1}-\left(n_{1}-p_{1}\right)$. Since $O_{n_{1}-p_{1}}^{\tilde{n}_{1}}(y) \in C^{M}(U)$, where $U$ is a compact neighborhood of the origin, $X_{i} \in C^{M+1}, i=1, \ldots, N$, it follows that $\omega_{k}^{1}(y)$ depends $C^{M}$-smoothly on $n_{1}-p_{1}$ columns. Hence, there exist smooth coefficients $b_{k, 1}(y), \ldots, b_{k, n_{1}-p_{1}}(y)$ with $\omega_{k}^{1}(y)=\sum_{l=1}^{n_{1}-p_{1}} b_{k, l}(y) w_{l}(y)$. Then if the columns $w_{1}(y), \ldots, w_{n_{1}-p_{1}}(y)$ correspond to the vectors $X_{i_{1}}(y), \ldots, X_{i_{n_{1}-p_{1}}}(y)$, and a column $v_{k}(y)$ corresponds to $X_{j_{k}}(y)$, then upon replacing $X_{j_{k}}(y)$ by

$$
X_{j_{k}}(y)-\sum_{l=1}^{n_{1}-p_{1}} b_{k, l}(y) X_{i_{l}}(y)
$$

we find that the part of the new column corresponding to $\omega_{k}^{1}(y)$ equals zero, where $k=1, \ldots, p_{1}$. Next, since at the points of $\chi_{p_{1} \ldots p_{M}}$ the columns $v_{1}(y), \ldots, v_{p_{1}}(y)$ belong to $\mathbb{R}^{n_{1}-p_{1}}$ we have $\omega_{k}^{2}(y)=0$ on $\chi_{p_{1} \ldots p_{M_{1}}}$. Apply the 
transformation (4.1) for all $k=1, \ldots, p_{1}$. In this case, each new column $v_{k}(y)$ vanishes at $y \in \chi_{p_{1} \ldots p_{M}}, k=1, \ldots, p_{1}$.

Thus, we obtain a new horizontal basis the nonzero images of whose vectors under $D \varphi$ at $\chi_{p_{1} \ldots p_{M}}$ are independent.

(ii) Next, we apply similar arguments to the subspace $H_{2}$ using the fact that we have already constructed a basis for $H_{1}$. Namely, at $y \in \chi_{p_{1} \ldots p_{M}}$, in each of $p_{2}-p_{1}$ vector fields, we delete the parts depending on $n_{2}-p_{2}$ linearly independent vector fields. This yields a new basis for $H_{2}$ the nonzero images of whose nonhorizontal vector fields independent and nonhorizontal (belonging to $\widetilde{H}_{2}$ ).

Continuing similarly, we obtain new bases for all $H_{k}, k=3, \ldots, M$. Denote these vector fields by $\left\{Y_{j}\right\}_{j=1}^{N}$.

Step IV. By step III, the intersection of $\chi_{p_{1} \ldots p_{M}}$ with the closure of every compact neighborhood $W \Subset U$ is closed. Indeed, take $\left\{y_{l}\right\} \rightarrow y$ as $l \rightarrow \infty$, where $y_{l} \in \chi_{p_{1} \ldots p_{M}} \cap \bar{W}$. Since $D \varphi\left(y_{l}\right)$ at each point $y_{l}$ vanishes on a certain collection of basis vector fields by the definition of $\chi_{p_{1} \ldots p_{M}}$, the continuity of the differential implies that $D \varphi(y)$ vanishes on the same vector fields and is non-degenerate on the rest since $W \Subset U$. Thus, the set $\chi_{p_{1} \ldots p_{M}}$ is measurable.

The measurability of $\chi_{p_{1} \ldots p_{M}}$ implies that almost all its points are density points (both with respect to $\mathcal{H}^{N}$ with balls in the Riemannian metric and $\mathcal{H}^{\nu}$ with Box $_{2}$-balls).

Step V. Estimate now the $\mathcal{H}^{N-\widetilde{N}}$-measure of the intersection of the level set passing through a density point $x \in \chi_{p_{1} \ldots p_{M}}$ of $\chi_{p_{1} \ldots p_{M}}$, and the ball $\operatorname{Box}_{2}(x, r)$. To this end, we use in the image and preimage the normal coordinates with respect to the points $\varphi(x)$ and $x$, and estimate the order of tangency between this level set and the tangent plane. We denote the resulting composition $\theta_{\varphi(x)}^{-1} \circ \varphi \circ \theta_{x}$ by $\psi$.

Evaluate $\psi$ at an arbitrary point $y=\sum_{i=1}^{N-\widetilde{N}} t_{i} Z_{j_{i}}(0)=\sum_{i=1}^{N-\widetilde{N}} t_{i} e_{j_{i}}$, where $Z_{j_{i}}=D \theta_{x}^{-1}\left[Y_{j_{i}}\right]$ are the basis vector fields tangent to the level set $\psi^{-1}(\psi(0))$ at zero. We have [NSW]

$$
\begin{aligned}
\psi(y)=\sum_{j=1}^{M}\left[\frac{1}{j !}\left(\sum_{i=1}^{N-\widetilde{N}} t_{i} Z_{j_{i}}\right)^{j} \psi\right](0) & +o\left(|y|^{M}\right) \\
& =\sum_{j=2}^{M}\left[\frac{1}{j !}\left(\sum_{i=1}^{N-\widetilde{N}} t_{i} Z_{j_{i}}\right)^{j} \psi\right](0)+o\left(|y|^{M}\right),
\end{aligned}
$$

where $o(\cdot)$ is uniform on $U$. Since 0 is a density point of zeroes of $D \psi Z_{j_{i}}$, the 
result of the action of every differential operator on it vanishes at 0 as well:

$$
\left(\sum_{i=1}^{N-\widetilde{N}} t_{i} Z_{j_{i}}\right)^{2} \psi(0)=0 .
$$

Similar statements hold for almost all (with respect to both $\mathcal{H}^{N}$ and $\mathcal{H}^{\nu}$ ) points of $\chi_{p_{1} \ldots p_{M}}$. Consequently, 0 is a density point of the zeroes of the function

$$
f(y)=\left(\sum_{i=1}^{N-\widetilde{N}} t_{i} Z_{j_{i}}\right)^{2} \psi(y) .
$$

Then we obtain

$$
\begin{aligned}
\psi(y)=\sum_{j=3}^{M}\left[\frac{1}{j !}\left(\sum_{i=1}^{N-\widetilde{N}} t_{i} Z_{j_{i}}\right)^{j} \psi\right](0) & +o\left(|y|^{M}\right) \\
& =\sum_{j=4}^{M}\left[\frac{1}{j !}\left(\sum_{i=1}^{N-\widetilde{N}} t_{i} Z_{j_{i}}\right)^{j} \psi\right](0)+o\left(|y|^{M}\right)
\end{aligned}
$$

since $\left(\sum_{i=1}^{N-\widetilde{N}} t_{i} Z_{j_{i}}\right)^{3} \psi(0)=0$.

Applying similar arguments, we arrive at $\psi(y)=o\left(|y|^{M}\right)$. This implies that the tangent plane approximates the level set up to $o\left(|y|^{M}\right)$. Indeed, take $y$ in the same level set as 0 , and the orthogonal projection $y^{\prime}$ of $y$ along $(\text { ker } D \psi(0))^{\perp}$ to the tangent plane. Then

$\psi(y)=\psi\left(y^{\prime}\right)+o\left(|y|^{M}\right)=\psi\left(y^{\prime}\right)+D \psi\left(y^{\prime}\right)\left(y-y^{\prime}\right)+o\left(\left|y-y^{\prime}\right|\right)=\psi\left(y^{\prime}\right)+C\left|y-y^{\prime}\right|$, and since $|C| \geq C_{0}>0$ uniformly on $U$, we have $\left|y-y^{\prime}\right|=o\left(|y|^{M}\right)$. Observe that here $o(\cdot)$ is uniform on $U$. Consequently, Theorem 3.7 yields

$$
\mathcal{H}^{N-\widetilde{N}}\left(\psi^{-1}(\psi(0)) \cap \operatorname{Box}_{2}(0, r)\right)=L r^{\nu-\nu_{0}},
$$

where $0<L_{1} \leq L \leq L_{2}<\infty$ uniformly on $U$, and

$$
\mathcal{H}^{N-\widetilde{N}}\left(\varphi^{-1}(\varphi(x)) \cap \operatorname{Box}_{2}(x, r)\right)=C_{x} r^{\nu-\nu_{0}},
$$

$\nu_{0}>\widetilde{\nu}$. Here the number $\nu_{0}$ is defined by the set $\chi_{p_{1} \ldots p_{M}}$, independently of $x$. Since the order of tangency is independent of the basis, the box in (4.2) is taken in the initial quasimetric $d_{2}$ which is constructed with respect to the initial basis $\left\{X_{i}\right\}_{i=1}^{N}$. Moreover, the relation

$$
\mathcal{H}^{N-\widetilde{N}}\left(\varphi^{-1}(\varphi(y)) \cap \operatorname{Box}_{2}(x, r)\right)=C_{y} r^{\nu-\nu_{0}}
$$


with $0<K_{1} \leq C_{y} \leq K_{2}<\infty$ holds for all points $y \in \chi_{p_{1} \ldots p_{M}} \backslash \Sigma_{\chi_{p_{1} \ldots p_{M}}}$ for some set $\Sigma_{\chi_{p_{1} \ldots p_{M}}}$ with $\mathcal{H}^{N}\left(\Sigma_{\chi_{p_{1} \ldots p_{M}}}\right)=\mathcal{H}^{\nu}\left(\Sigma_{\chi_{p_{1} \ldots p_{M}}}\right)=0$.

Step VI. Suppose that $z \in \widetilde{\mathbb{M}}$. Estimate the measure

$$
\mathcal{H}^{\nu-\widetilde{\nu}}\left(\varphi^{-1}(z) \cap\left(\chi_{p_{1} \ldots p_{M}} \backslash \Sigma_{\chi_{p_{1} \ldots p_{M}}}\right) \cap W\right)
$$

for $W \Subset U$. Since $d_{2}$ and $d_{c c}$ are locally equivalent, the conditions of $5 r$ covering lemma are fulfilled for the balls centered at the points of $\chi_{p_{1} \ldots p_{M}} \backslash$ $\Sigma_{\chi_{p_{1} \ldots p_{M}}}$. Consider a covering $\bigcup_{i \in \mathbb{N}} \operatorname{Box}_{2}\left(x_{i}, r_{i}\right)$ of the set

$$
\varphi^{-1}(z) \cap\left(\chi_{p_{1} \ldots p_{M}} \backslash \Sigma_{\chi_{p_{1} \ldots p_{M}}}\right) \cap W
$$

such that the balls $\left\{\operatorname{Box}_{2}\left(x_{i}, r_{i} / 5 l\right)\right\}_{i \in \mathbb{N}}$ are disjoint. Here the number $l$ depends on the constants in the equivalence of $d_{2}$ and $d_{c c}$. Then we have

$$
\begin{aligned}
& \mathcal{H}_{\delta}^{\nu-\widetilde{\nu}}\left(\varphi^{-1}(z) \cap\left(\chi_{p_{1} \ldots p_{M}} \backslash \Sigma_{\chi_{p_{1} \ldots p_{M}}}\right) \cap W\right) \\
& \leq C \delta^{\nu_{0}-\widetilde{\nu}} \sum_{i \in \mathbb{N}} r_{i}^{\nu-\nu_{0}} \leq C_{1} \delta^{\nu_{0}-\widetilde{\nu}} \sum_{i \in \mathbb{N}} \mathcal{H}^{N-\widetilde{N}}\left(\varphi^{-1}(\varphi(x)) \cap \operatorname{Box}_{2}\left(x_{i}, r_{i}\right)\right) \\
& \leq C_{2} \delta^{\nu_{0}-\widetilde{\nu}} \sum_{i \in \mathbb{N}} \mathcal{H}^{N-\widetilde{N}}\left(\varphi^{-1}(\varphi(x)) \cap \operatorname{Box}_{2}\left(x_{i}, r_{i} / 5 l\right)\right) \leq \delta^{\nu_{0}-\widetilde{\nu}} L \rightarrow 0
\end{aligned}
$$

as $\delta \rightarrow 0$ since $L \leq \mathcal{H}^{N-\widetilde{N}}\left(\varphi^{-1}(z) \cap\left(\chi_{p_{1} \ldots p_{M}} \backslash \Sigma_{\chi_{p_{1} \ldots p_{M}}}\right) \cap W\right)$ is independent of $\delta>0$. Thus, the intersection of every level set with $\chi_{p_{1} \ldots p_{M}} \backslash \Sigma_{\chi_{p_{1} \ldots p_{M}}}$ has $\mathcal{H}^{\nu-\widetilde{\nu}}$-measure zero. Consequently,

$$
\mathcal{H}^{\nu-\widetilde{\nu}}\left(\varphi^{-1}(z) \cap(\chi \backslash \Sigma)\right)=0
$$

where $\Sigma=\bigcup_{p_{1} \ldots p_{M}} \Sigma_{\chi_{p_{1} \ldots p_{M}}}$ and $\mathcal{H}^{N}(\Sigma)=\mathcal{H}^{\nu}(\Sigma)=0$. Now the theorem follows from [Fe2, 2.10.25].

\section{The Degeneration Set}

Theorem 5.1. For $\mathcal{H}^{\tilde{\nu}}$-almost all $t \in \widetilde{\mathbb{M}}$ we have

$$
\mathcal{H}^{\nu-\tilde{\nu}}\left(\varphi^{-1}(t) \cap Z\right)=0 \text {. }
$$

Proof. Step I. We have $Z=Z_{1} \cup Z_{2}$, where $\mathcal{H}^{\widetilde{N}}\left(\varphi\left(Z_{1}\right)\right)=\mathcal{H}^{\tilde{\nu}}\left(\varphi\left(Z_{1}\right)\right)=0$, and $\mathcal{H}^{N-\widetilde{N}}\left(\varphi^{-1}(t) \cap Z_{2}\right)=0$ for all $t \in \widetilde{\mathbb{M}}$ (see, for instance, [Km6]). 
Step II. Note that $\mathcal{H}^{\widetilde{N}}\left(\varphi\left(Z_{2}\right)\right)<\infty$ since otherwise we can represent it as a countable union of sets of finite measure. Next, decompose $Z_{2}$ as $\bigcup_{i=0}^{\widetilde{N}-1} C_{i}$, where $\operatorname{rank} D \varphi(x)=i$ for $x \in C_{i}$.

Fix $0 \leq i \leq \widetilde{N}-1$. Without loss of generality we may assume that $C_{i}$ is a compact set. Consider a compact set $\widetilde{C}_{i} \subset C_{i}$ such that $\varphi\left(\widetilde{C}_{i}\right)$ is a compact subset of the set of density points of $\varphi\left(C_{i}\right)$ and $\mathcal{H}^{N}\left(\varphi\left(C_{i}\right) \backslash \varphi\left(\widetilde{C}_{i}\right)\right)<\varepsilon$ for a fixed arbitrary $\varepsilon>0$. Consider the mapping

$$
\xi_{i}(x)=\left(\varphi(x), x_{N-i+1}, \ldots, x_{N}\right): \mathbb{R}^{N} \rightarrow \mathbb{R}^{\widetilde{N}} \times \mathbb{R}^{N-i} .
$$

Observe that $\xi_{i}\left(C_{i}\right)$ is an $N$-rectifiable set. Our assumption implies that $\xi_{i}$ is locally bi-Lipschitz on $C_{i}$. Consequently, $\mathcal{H}^{N-\widetilde{N}}\left(\xi_{i}\left(\varphi^{-1}(t) \cap \widetilde{C}_{i}\right)\right)=0$ for all $t \in \widetilde{\mathbb{M}}$. Consider the mapping $\pi: \xi\left(C_{i}\right) \rightarrow \mathbb{R}^{\widetilde{N}}$ defined as

$$
\pi\left(x_{1}, \ldots, x_{\widetilde{N}+N-i}\right)=\left(x_{1}, \ldots, x_{\widetilde{N}}\right) .
$$

Apply the classical coarea formula to $\pi, C_{i}$ and $\widetilde{C}_{i}$ :

$$
\begin{aligned}
\int_{\xi\left(C_{i}\right)} \mathcal{J}(\pi, x) d \mathcal{H}^{N}(x) & \geq \int_{\xi\left(C_{i}\right)} \chi_{\xi\left(\widetilde{C}_{i}\right)}(x) \mathcal{J}(\pi, x) d \mathcal{H}^{N}(x) \\
& =\int_{\varphi\left(C_{i}\right)} d \mathcal{H}^{\widetilde{N}}(t) \int_{\pi^{-1}(t) \cap \xi\left(C_{i}\right)} \chi_{\xi\left(\widetilde{C}_{i}\right)}(u) d \mathcal{H}^{N-\widetilde{N}}(u) .
\end{aligned}
$$

By the definition of $\widetilde{C}_{i}$, we find that $\mathcal{J}(\pi, x)=1$ for $x \in \xi\left(\widetilde{C}_{i}\right)$. Furthermore,

$$
\mathcal{H}^{N-\widetilde{N}}\left(\pi^{-1}(t) \cap \xi\left(C_{i}\right)\right)=\mathcal{H}^{N-\widetilde{N}}\left(\xi_{i}\left(\varphi^{-1}(t) \cap \widetilde{C}_{i}\right)\right)=0 .
$$

It follows from (5.1) that $\mathcal{H}^{N}\left(\xi\left(\widetilde{C}_{i}\right)\right)=0$. Finally, have

$$
\mathcal{H}^{N}\left(\widetilde{C}_{i}\right)=\mathcal{H}^{\nu}\left(\widetilde{C}_{i}\right)=0
$$

because $\xi_{i}$ is bi-Lipschitz. Since $\varepsilon>0$ is arbitrary, there exists a set $\Sigma_{i}$ of measure zero in $\varphi\left(C_{i}\right)$ such that

$$
\mathcal{H}^{N}\left(\varphi^{-1}\left(\varphi\left(C_{i}\right) \backslash \Sigma_{i}\right) \cap C_{i}\right)=\mathcal{H}^{\nu}\left(\varphi^{-1}\left(\varphi\left(C_{i}\right) \backslash \Sigma_{i}\right) \cap C_{i}\right)=0 .
$$

Therefore, $\mathcal{H}^{\nu}\left(\bigcup_{i=0}^{\widetilde{N}-1} \varphi^{-1}\left(\varphi\left(C_{i}\right) \backslash \Sigma_{i}\right) \cap C_{i}\right)=0$. 
Step III. Since $\varphi$ is contact, it is locally Lipschitz with respect to every sub-Riemannian metric. Reasoning as in [Fe2, Theorem 2.10.25], we obtain

$$
\begin{aligned}
& 0 \leq \int_{\widetilde{\mathbb{M}}} d \mathcal{H}^{\tilde{\nu}}(t) \int \\
& \varphi^{-1}(t) \cap \bigcup_{i=0}^{\tilde{N}-1} \varphi^{-1}\left(\varphi\left(C_{i}\right) \backslash \Sigma_{i}\right) \cap C_{i} \\
& \leq C(\operatorname{Lip}(\varphi), \nu, \tilde{\nu}) \mathcal{H}^{\nu}\left(\bigcup_{i=0}^{\tilde{N}-1} \varphi^{-1}\left(\varphi\left(C_{i}\right) \backslash \Sigma_{i}\right) \cap C_{i}\right)=0 .
\end{aligned}
$$

Furthermore, the $\mathcal{H}^{\widetilde{N}}$-measure zero set $\bigcup_{i=0}^{\widetilde{N}-1} \Sigma_{i}$ is included into an $\mathcal{H}^{\widetilde{N}}$-measure zero Borel set $\Sigma$. Then, its preimage $\varphi^{-1}(\Sigma)$ is also a Borel set, and the set $\varphi^{-1}(\Sigma) \cap Z_{2}$ is measurable. By the definition,

$$
\mathcal{H}^{\widetilde{N}}\left(\varphi\left(\varphi^{-1}(\Sigma) \cap Z_{2}\right)\right)=\mathcal{H}^{\widetilde{\nu}}\left(\varphi\left(\varphi^{-1}(\Sigma) \cap Z_{2}\right)\right)=0 .
$$

combining the results, we deduce that

$$
\int_{\widetilde{\mathbb{M}}} d \mathcal{H}^{\tilde{\nu}}(t) \int_{\varphi^{-1}(t) \cap Z} d \mathcal{H}^{\nu-\tilde{\nu}}(u)=0,
$$

and thus we complete the proof.

\section{The Coarea Formula}

Theorem 6.1 (Coarea Formula). If a contact mapping $\varphi: \mathbb{M} \rightarrow \widetilde{\mathbb{M}}$ satisfies Assumption 2.26 then the coarea formula holds:

$$
\int_{\mathbb{M}} \mathcal{J}_{\widetilde{N}}^{S R}(\varphi, x) d \mathcal{H}^{\nu}(x)=\int_{\widetilde{\mathbb{M}}} d \mathcal{H}^{\tilde{\nu}}(t) \int_{\varphi^{-1}(t)} d \mathcal{H}^{\nu-\tilde{\nu}}(u) .
$$

Proof. Obvserve that $\mathbb{M}=\mathbb{D} \sqcup \chi \sqcup Z$. Consequently,

$$
\begin{aligned}
\int_{\mathbb{M}} \mathcal{J}_{\widetilde{N}}^{S R}(\varphi, x) d \mathcal{H}^{\nu}(x)= & \int_{\mathbb{D}} \mathcal{J}_{\widetilde{N}}^{S R}(\varphi, x) d \mathcal{H}^{\nu}(x) \\
& +\int_{\chi} \mathcal{J}_{\widetilde{N}}^{S R}(\varphi, x) d \mathcal{H}^{\nu}(x)+\int_{Z} \mathcal{J}_{\widetilde{N}}^{S R}(\varphi, x) d \mathcal{H}^{\nu}(x) .
\end{aligned}
$$


Theorem 3.21 yields

$$
\int_{\mathbb{D}} \mathcal{J}_{\widetilde{N}}^{S R}(\varphi, x) d \mathcal{H}^{\nu}(x)=\int_{\widetilde{\mathbb{M}}} d \mathcal{H}^{\tilde{\nu}}(t) \int_{\varphi^{-1}(t) \cap \mathbb{D}} d \mathcal{H}^{\nu-\tilde{\nu}}(u) .
$$

Theorem 4.1, implies

$$
\int_{\chi} \mathcal{J}_{\widetilde{N}}^{S R}(\varphi, x) d \mathcal{H}^{\nu}(x)=0=\int_{\widetilde{\mathbb{M}}} d \mathcal{H}^{\tilde{\nu}}(t) \int_{\varphi^{-1}(t) \cap \chi} d \mathcal{H}^{\nu-\tilde{\nu}}(u) .
$$

Finally, since $\mathcal{J}_{\widetilde{N}}^{S R}(\varphi, x)=0$ on $Z$, Theorem 5.1 yields

$$
\int_{Z} \mathcal{J}_{\widetilde{N}}^{S R}(\varphi, x) d \mathcal{H}^{\nu}(x)=0=\int_{\widetilde{\mathbb{M}}} d \mathcal{H}^{\tilde{\nu}}(t) \int_{\varphi^{-1}(t) \cap Z} d \mathcal{H}^{\nu-\tilde{\nu}}(u) .
$$

Combinig these relations, we obtain (6.1). The theorem follows.

\section{References}

[AgMa] Agrachev A., Marigo A. Nonholonomic tangent spaces: intrinsic construction and rigid dimensions, Electron. Res. Announc. Amer. Math. Soc. 9 (2003), 111-120.

[AmKi] Ambrosio L., Kirchheim B. Rectifiable sets in metric and Banach spaces, Math. Ann. 318 (2000), 527-555.

[Be] Bellaïche A. Tangent Space in Sub-Riemannian Geometry, In: SubRiemannian geometry. Basel, Birkhauser Verlag, 1996, 1-78.

[BLU] Bonfiglioli A., Lanconelli E., Uguzzoni F. Stratified Lie Groups and Potential Theory for Their Sub-Laplacians, Springer, 2007.

[1] Chow W. L., Über Systeme von linearen partiellen Differentialgleichungen erster Ordung, Math. Ann. 117 (1939), 98-105.

[CS] Citti G., Sarti A. A cortical based model of perceptual completion in the roto-translation space, Lecture Notes of Seminario Interdisciplinare di Matematica 3 (2004), 145-161.

[EG] Evans L. C., Gariepy R. F. Measure theory and fine properties of functions, CRC Press, Boca Raton, 1992. 
[Fe1] Federer H. Curvature measures, Trans. Amer. Math. Soc. 93 (1959), 418-491.

[Fe2] Federer H. Geometric Measure Theory, NY: Springer, 1969.

[FeFl] Federer H., Fleming W. H. Normal and Integral Currents, Ann. Math. 72 (2) (1960), 458-520.

[FoSt] Folland G. B., Stein E. M. Hardy spaces on homogeneous groups, Princeton Univ. Press, 1982.

[GMS] Giaquinta M., Modica G., Souček J. Cartesian currents in the calculus of variations, V. I, II, Springer-Verlag, Berlin, 1998.

[Gr] Gromov M. Carnot-Carathéodory Spaces Seen From Within, In: Sub-Riemannian geometry. Basel, Birkhauser Verlag, 1996, 79-318.

[He] Heinonen J. Calculus on Carnot groups, In: Fall school in analysis (Jyväskylä, 1994). Jyväskylä, University of Jyväskylä, 1994, 1-32.

[HlPa] Hladky R. K., Pauls S. D. Minimal surfaces in the roto-translation group with applications to a neuro-biological image completion model, arXiv:math.DG/0509636, 27 Sep. 2005.

[Je] Jean F. Uniform estimation of sub-riemannian balls, Journal on Dynamical and Controle Systems 7 (4) (2001), 473-500.

[Km1] Karmanova M. B. Metric Differentiability of Mappings and Geometric Measure Theory, Doklady Mathematics 71 (2) (2005), 224-227.

[Km2] Karmanova M. B. Rectifiable Sets and Coarea Formula for MetricValued Mappings, Doklady Mathematics, 73 (3) (2006), 323-327.

[Km3] Karmanova M. B. Metric Rademacher Theorem and Area Formula for Metric-Valued Lipschitz Mappings, Vestnik NSU 6 (4) (2006), 50-69.

[Km4] Karmanova M. Formulas of Geometric Measure Theory on Rectifiable Metric Spaces, Contemporary Mathematics 424 (2007), 103136.

[Km5] Karmanova M. B. Area and Coarea Formulas for Sobolev Mappings with Values in a Metric Space, Siberian Mathematical Journal 48 (4) (2007), 778-788. 
[Km6] Karmanova M. Rectifiable Sets and Coarea Formula for MetricValued Mappings, Journal of Functional Analysis 254 (5) (2008), 1410-1447.

[Km7] Karmanova M. B. Characteristic Set of Smooth Mappings of Carnot Manifolds, Dokl. AN 425 (3) (2009), 314-319.

[Km8] Karmanova M. B. A New Approach to Investigation of CarmotCarathéodory Geometry, Doklady Mathematics, 82 (2) (2010), 746750 .

[Km9] Karmanova M. The New Approach to Investigation of CarnotCarathéodory Geometry, GAFA (2011), accepted.

[Km10] Karmanova M. B. Convergence of Scaled Vector Fields and Local Approximation Theorem on Carnot-Carathéodory Spaces and Applications, Dokl. AN 440 (6) (2011), 736-742.

[KmVod] Karmanova M., Vodopyanov S. Geometry of Carnot-Carathéodory Spaces, Differentiability, Coarea and Area Formulas, In: Analysis and Mathematical Physics. Birkhäuser, 2009. P. 233-335.

[Ki] Kirchheim B. Rectifiable metric spaces: local structure and regularity of the Hausdorff measure, Proc. AMS. 121 (1994), 113-123.

[Kr] Kronrod A. S. On functions of two variables (in Russian), Uspehi Matem. Nauk (N. S.) 5 (1950), 24-134.

[LiYa] F. Lin and X. Yang, Geometric measure theory, Science Press, Beijing a. o., 2002.

[Mg1] Magnani V. Elements of Geometric Measure Theory on subRiemannian groups, Tesi di Perfezionamento. Pisa: Scuola Normale Superiore (Thesis), 2002.

[Mg2] Magnani V. Blow-up of regular submanifolds in Heisenberg groups and applications, Cent. Eur. J. Math. 4 (1) (2006), 82-109.

[MrMo1] Margulis G. A., Mostow G. D. The differential of quasi-conformal mapping of a Carnot-Carathéodory space, Geometric and Functional Analysis 5 (2) (1995), 402-433.

[MrMo2] Margulis G. A., Mostow G. D. Some remarks on the definition of tangent cones in a Carnot-Carathéodory space, Journal D'Analyse Math. 80 (2000), 299-317. 
[Mn] Montgomery R. A Tour of Subriemannian Geometries, Their Geodesics and Applications, Providence, AMS, 2002.

[NSW] Nagel A., Stein E. M., Wainger S. Balls and metrics defined by vector fields I: Basic properties, Acta Math. 155 (1985), 103-147.

[Ot] Ohtsuka M. Area Formula, Bull. Inst. Math. Acad. Sinica 6 (2) (2) (1978), 599-636.

[Pa] Pansu P. Geometrie du group d'Heisenberg, Univ. Paris VII, 1982.

[Po] Postnikov M. M. Lectures in Geometry. Semester V: Lie Groups and Lie Algebras, Moscow, "Nauka", 1982.

[2] Rashevsky P. K., Any two points of a totally nonholonomic space may be connected by an admissible line, Uch. Zap. Ped. Inst. im. Liebknechta. Ser. Phys. Math. 2 (1938), 83-94.

[RoSt] Rothschild L. P., Stein E.M. Hypoelliptic differential operators and nilpotent groups, Acta Math. 137 (1976), 247-320.

[Vod1] Vodopyanov S. K. The Theory of Lebesgue Integral: Lecture Notes in Calculus, Novosibirsk State University Press, Novosibirsk, 2006.

[Vod2] Vodop'yanov S. K. Differentiability of Curves in Carnot Manifold Category, Dokl. AN 410 (4) (2006), 1-6.

[Vod3] Vodopyanov S. K. Differentiability of mappings of Carnot Manifolds and Isomorphism of Tangent Cones, Doklady Mathematics 74 (3) (2006), 844-848.

[Vod4] Vodopyanov S. Geometry of Carnot-Carathéodory Spaces and Differentiability of Mappings, Contemporary Mathematics 424 (2007), 247-301.

[Vod5] Vodopyanov S. K. Differentiability of Mappings in the Geometry of Carnot Manifolds, Sib. Math. Zh., 48 (2) (2007), 251-271.

[VodG] Vodop'yanov S. K., Greshnov A. V. On the Differentiability of Mappings of Carnot-Carathéodory Spaces, Doklady Mathematics 67 (2) (2003), 246-250.

[VodKm1] Vodopyanov S. K., Karmanova M. B. Local Geometry of Carnot Manifolds Under Minimal Smoothness, Doklady Mathematics, 75 (2) (2007), 240-246. 
[VodKm2] Vodopyanov S. K., Karmanova M. B. Local Approximation theorem in Carnot Manifolds Under Minimal Assumptions on smoothness, Doklady Mathematics, 80 (1) (2009), 585-589.

[VodU] Vodopyanov S. K., Ukhlov A. D. Set functions and their applications in the theory of Lebesgue and Sobolev spaces, I: Siberian Adv. Math. 14 (4) (2004), 78-125; II: Siberian Adv. Math. 15 (1) (2005), 91-125. 